\title{
On matroids that are transversal and cotransversal
}

Meenu Mariya Jose

Supervisor: Dr. Dillon Mayhew

\section{VICTORIA UNIVERSITY OF WELLINGTON \\ Te Herenga Waka

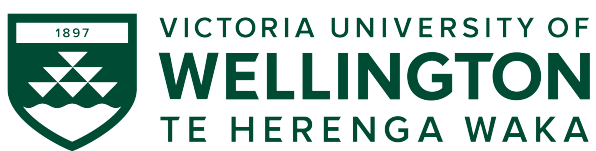 \\ School of Mathematics and Statistics \\ Te Kura Mātai Tatauranga}

\begin{abstract}
A thesis
submitted to the Victoria University of Wellington in fulfilment of the requirements for the degree of Doctor of Philosophy in Mathematics.
\end{abstract}

Victoria University of Wellington 2020 



\begin{abstract}
There are distinct differences between classes of matroids that are closed under principal extensions and those that are not. Finite-field-representable matroids are not closed under principal extensions and they exhibit attractive properties like well-quasi-ordering and decidable theories (at least for subclasses with bounded branch-width). Infinite-field-representable matroids, on the other hand, are closed under principal extensions and exhibit none of these behaviours. For example, the class of rank-3 real representable matroids is not well-quasi-ordered and has an undecidable theory. The class of matroids that are transversal and cotransversal is not closed under principal extensions or coprincipal coextentions, so we expect it to behave more like the class of finite-field-representable matroids. This thesis is invested in exploring properties in the aforementioned class.
\end{abstract}

A major idea that has inspired the thesis is the investigation of well-quasi-ordered classes in the world of matroids that are transversal and cotransversal. We conjecture that any minor-closed class with bounded branch-width containing matroids that are transversal and cotransversal is well-quasi-ordered. In Chapter 8 of the thesis, we prove this is true for lattice-path matroids, a well-behaved class that falls in this intersection.

The general class of lattice-path matroids is not well-quasi-ordered as it contains an infinite antichain of so-called 'notch matroids'. The interesting phenomenon that we observe is that this is essentially the only antichain in this class, that is, any minor-closed family of lattice-path matroids that contains only finitely many notch matroids is well-quasi-ordered. This answers a question posed by Jim Geelen. 
Another question that drove the research was recognising fundamental transversal matroids, since these matroids are also cotransversal. We prove that this problem in general is in NP and conjecture that it is NP-complete. We later explore this question for the classes of lattice-path and bicircular matroids. We are successful in finding polynomial-time algorithms in both classes that identify fundamental transversal matroids. We end this part by investigating the intersection of bicircular and cobicircular matroids. We define a specific class - whirly-swirls and conjecture that eventually any matroid in the above mentioned intersection belongs to this class. 


\section{Acknowledgements}

This is not a thesis that was created in three years but rather, it is the result of the last twenty six years. And it has taken a village to create this. I am infinitely grateful for the divine providence that has always arranged miracles in wondrous serendipity. I am grateful to be here right now.

I thank Dr. Dillon Mayhew, my supervisor and mentor. I could not have asked for a better guide. He is a perfect amalgam of a researcher, teacher, presenter and editor (and poker player). I admire the ease with which he communicates difficult mathematical ideas in lucid terms to people of all ages. Thank you for being so considerate and very supportive of whatever I choose to do.

My parents, Rosy and Jose, and my brother Jithu - I cannot even begin to thank you. You are my home regardless of where we are. You have always helped me stay mindful of my individuality while reminding me that I am part of something bigger. Thank you for staying committed to help me fulfill my dreams even at times when it was not easy to do so. Prasantho, you are the best mirror I have found. Thank you for being my best friend and my person that I can grow with.

I am ever grateful for my friendships that have aged like wine and have supported me through everything no matter how far apart we have been. Christy, you are the first person I call about anything under the sun. Priya and Manika, the best people from my Delhi days and the reason I want to visit again and again. And my trio at CUSAT - Lakshmi and Nusrath - who have added - and still do - so much color to my life. And my Chakkakurus - you are my rebellious girl group who make me cry with laughter every single time.

My tribe at Vic cannot be thanked enough, especially Jasmine, Susan, Jessica, Will and Emma. I did not expect to forge such rich friendships before coming here. Each one of you have added such warmth and depth 
to my experience here. I also thank Ellis, Del, Linus and Virginia for being part of my Vic family. I am deeply grateful to have you as my roommate Babitha. Thank you for the endless discussions, fun cooking time and most of all, being part of my home here. You all have given me a space in your heart so much so that Wellington feels like a second home. That experience is not complete without Tony chettan and family. Whenever I leave your house, it is with a satiated heart and belly.

There are family members who have supported and nourished me along the way. My grandparents - Mariyam, Ittoop, Poulose and Elsy, Peppan(Tony), Preetha Aunty, Aaron, Anna, Justin, Treesa velyammachi, Momo and Rani aunty. Thank you.

Two teachers who did not lose faith in me when I was not sure - Ms. Nandita Narain (our beloved Nandy) and Dr. A. Vijayakumar. I am deeply indebted to you both for pushing me the right amount. And there are other teachers who have inspired me to choose teaching - Nishi Miss, Neena Ma'am, Swapna Miss, Lakshmi Ma'am, Sabu Sir, Jyoti Miss, Lakshmy Ma'am, Binu Miss and Kiran Sir.

I would like to thank the School of Mathematics and Statistics for providing me with a scholarship and being supportive and encouraging. Especially Ginny, Steven, Geoff and Caitlin. I would like to thank my counselor Shiranthi for holding space during some dark days.

I would like to thank Joe Bonin for insights about lattice path matroids and Jim Geelen for asking a great question. A special thanks to Jasmine and Priya for reading drafts of my thesis and to Susan for offering to do so. I am grateful to James Oxley for giving thoughtful feedback in presenting mathematics.

As the cliche goes, last but not the least, I would like to thank myself for being committed to staying open and curious. 


\section{Contents}

$\begin{array}{lll}1 & \text { Introduction } & 1\end{array}$

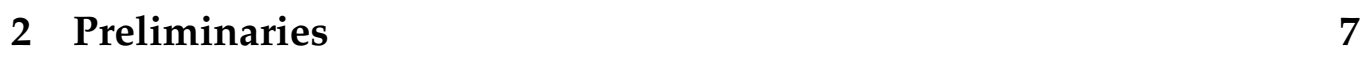

2.1 Transversal matroids . . . . . . . . . . . . . . . 7

2.2 Fundamental transversal matroids . . . . . . . . . . . . . 10

2.3 Lattice-path matroids . . . . . . . . . . . . . . . . . 13

2.4 Bicircular matroids . . . . . . . . . . . . . . 17

2.5 Algorithms and Complexity . . . . . . . . . . . . . . . 19

I Definability and Recognisability 21

3 Monadic second-order Logic 25

$4 \quad$ Definability in classes of transversal matroids 31

4.2 Defining lattice-path matroids . . . . . . . . . . . . . . . 31

4.3 Defining fundamental matroids . . . . . . . . . . . . 35

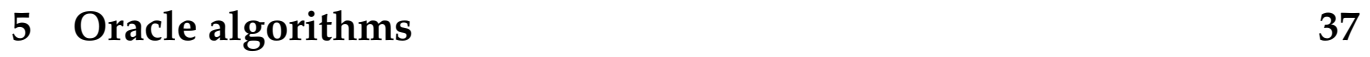

II Well-quasi-ordering 44

\begin{tabular}{|lll}
6 & Well-quasi-ordering & 45
\end{tabular} 
7 Are lattice path matroids well-quasi-ordered? 49

8 Notch matroids and Well-Quasi-Ordering 65

III Fundamental and bicircular matroids 69

$9 \quad$ The problem FUNDAMENTAL TRANSVERSAL MATROID 73

$\begin{array}{ll}10 \text { Recognising fundamental lattice path matroids } & 77\end{array}$

\begin{tabular}{ll}
\hline 1 Fundamental bicircular matroids & 87
\end{tabular}

$\begin{array}{ll}12 \text { Bicircular and cobicircular matroids } & 105\end{array}$

\begin{tabular}{ll}
\hline Bibliography & 111
\end{tabular} 


\section{Chapter 1}

\section{Introduction}

Representable matroids have been in the limelight since the inception of matroid theory by Hassler Whitney in 1935 [7]. There is a vast literature to show that finite-field matroids are well behaved and infinite-field matroids are not. For example, finite-field matroids of bounded branch-width exhibit the well-quasi-ordering property under the minor relation [15]. This result has now been proved without the restriction on branch-width (although the proof is not published) by the same authors. The same cannot be said for infinite fields. Also, any minor-closed class of $F$-representable matroids, where $F$ is a finite field, has finitely many excluded minors [14]. This statement was recently proved by Geelen, Gerards, and Whittle, and includes as a special case Rota's famous conjecture. If $F$ is an infinite field, then the anti-chain of excluded minors is not only infinite, but maximal [24]. This means that every F-representable matroid is contained as a minor in an excluded minor for the class. For another example of the contrast between these classes, we consider the property of being characterisable in a logical language. If $F$ is finite, the class of $F$-representable matroids can be characterised in monadic second order logic whereas if $F$ is infinite, this is not true [25].

Furthermore finite-field-matroids of bounded branchwidth have a decidable monadic-second-order theory whereas infinite-field-matroids have an undecidable theory, even for matroids of rank 3 (and hence branch-width 3) [12]. This points in the direction that finite-field matroids lie at the 'nice' end of a spectrum, and infinite-field matroids lie at the 
'bad' end. The question that got us interested is where the matroids that are transversal and cotransversal lie? We conjecture it is in the middle, but closer to 'nice' than to 'bad'.

Another property that can help categorise matroids is being closed under principal extensions. There is a clear distinction between classes that are closed under principal extensions, and those that are not. Finite-field-representable matroids are not closed under principal extensions and, you guessed it right, infinite-field-representable matroids are. This is at the heart of the proof that the anti-chain of excluded minors for real representability is maximal, as recognised by Matus [23].

Transversal matroids were first investigated by Edmonds and Fulkerson [10] and soon proved to be an important class of matroids contributing both to transversal theory and matroid theory. That said, the class of transversal and cotransversal matroids remains quite mysterious. Testing the membership of this class is non-trivial. In particular, we do not know of a polynomial-time algorithm to decide if a transversal matroid is cotransversal. We observe that the class of transversal matroids is not closed under principal extensions, but is closed under co-principal co-extensions. Hence, we suspect that they behave more like infinite-field-matroids and rightly so. Note that corank-3 transversal matroids (which therefore have branch-width at most 3) are not well-quasi-ordered, and have an undecidable monadic theory. This implies transversal matroids behave badly, and so do cotransversal. However, we think the intersection of these classes is well-behaved. This is supported by the fact that the class of matroids that is transversal and cotransversal is not closed under principal extensions or co-principal co-extensions. We delve deeper into this idea by exploring classes that fall in this intersection.

The class that forms the intersection of transversal and cotransversal matroids is not well-quasi-ordered. However, we believe the following to be true:

Conjecture 1.1.1. Any minor-closed class with bounded branch-width containing only matroids that are transversal and cotransversal is well-quasi-ordered. 
We prove this is true for the class of lattice-path matroids in Chapter 7 Lattice-path matroids are matroids that are transversal and cotransversal. They can be easily visualised as lattice-path presentations that are made of paths in the integer lattice (Figure 1.1). Each element in the matroid is a 'staircase' in the presentation.

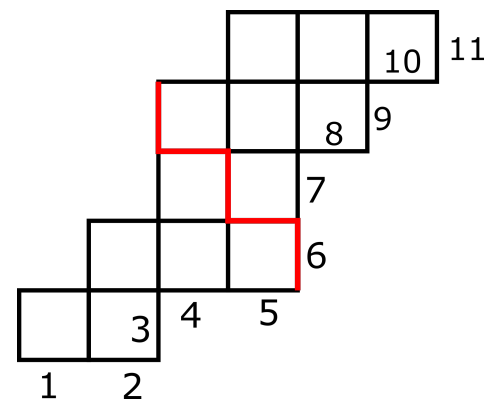

Figure 1.1: A lattice-path presentation where the corresponding matroid has 11 elements. The staircase in red corresponds to element 6.

Their rich structural properties led us to an elegant proof of the following theorem:

Theorem 1.1.2. Any class of lattice-path matroids with bounded branch-width is well-quasi-ordered.

A second class of matroids that spiked our interest was the the class of bicircular matroids. They arise from graphs similar to graphic matroids but differ in the set of circuits. While the circuits of graphic matroids are the cycles in the graph, the dependent sets of bicircular matroids are minimal connected sets of edges that have at least two cycles. By virtue of arising from graphs, the class of bicircular matroids is also well-quasi-ordered even though the minor relation varies slightly. This result is illustrated in Chapter 6

Another property that can be used to measure the richness of a class is being pigeonhole. If each matroid in a class can be decomposed by a ternary tree in such a way that only a bounded amount of information flows across displayed separations, then the class has bounded decomposition-width. A class is said to be pigeonhole if every subclass with bounded branch-width also has bounded decomposition-width [12]. 
Assume $U$ is a $k$-separating set. Let $X$ and $X^{\prime}$ be subsets of $U$. We say $X$ is equivalent to $X^{\prime}$ if and only if $X \cup Z$ is independent implies $X^{\prime} \cup Z$ is independent, for all $Z \subseteq E(M)-U$.

If for every $k$, there is a number $f(k)$ such that there are at most $f(k)$ equivalence classes in a $k$-separating set $U$, then the class of matroids has the strong pigeonhole property. Again, any class of finite-field-representable matroids is strongly pigeonhole. The property of being strongly pigeonhole is stricter than being pigeonhole and the former implies the latter. We now suspect the following is true and hence conjecture:

Conjecture 1.1.3. The class of transversal and cotransversal matroids is strongly pigeonhole.

This has been proved for the class of fundamental transversal matroids in [13]. Classes with the strong pigeonhole property tend to have good algorithmic properties as is illustrated by the next conjecture:

Conjecture 1.1.4. A class of tranversal and cotransversal matroids with bounded branch width has a decidable monadic-second-order theory.

A theory is a set of sentences - closed formulas - that is closed under logical consequences. A theory is decidable if there is a polynomial-time algorithm to determine whether a given formula belongs to the theory.

Fundamental transversal matroids are prime examples of matroids that are transversal and cotransversal and it only seems natural to question when transversal matroids are fundamental. We find that this problem is in NP and conjecture that it is NP complete. Note that the matroid is input as a corresponding bipartite graph.

That said, we find that for the subclasses of lattice-path and bicircular matroids, there exist polynomial-time algorithms to determine whether the given matroid is fundamental.

These conjectures have directed this thesis. Even though they remain conjectures at the end of the thesis, we have made ample progress in understanding the class of fundamental tranversal matroids and hope to have shed some light on its interesting behaviour. 


\subsubsection{Structure of thesis}

In Chapter 2, we introduce and explain background information necessary for a smooth reading of the thesis. The thesis is then divided into three parts. The first part explores the concepts of definability and recognisability in the context of transversal and cotransversal matroids. Lattice-path matroids are defined in monadic second order logic with rank (denoted as $\mathrm{MS}_{0}^{+}$) and fundamental transversal matroids are defined in monadic second order logic (denoted as $M S_{0}$ ) in the initial chapters. As a result, we have the two following theorems:

Theorem 1.1.5. Lattice-path matroids are axiomatisable in $M S_{0}^{+}$.

Theorem 1.1.6. Fundamental matroids are axiomatisable in $M S_{0}$.

In the last chapter of this part, we summarise the recognisability via oracle algorithms of all the classes considered in this thesis.

In the second part, we investigate the property of well-quasi-ordering and first show that bicircular matroids exhibit this pattern by virtue of arising from graphs. We then prove that though lattice-path matroids do not form a well-quasi-ordered class, certain restrictions help model this behaviour. We introduce the notion of 'square-width' which is almost the same as branch-width for lattice-path matroids. This notion provided us with the following result:

Theorem 1.1.7. The class of lattice-path matroids of bounded branch-width is well-quasi-ordered.

Notch matroids form a subclass of lattice-path matroids where the lower bounding path in the presentation has at most one 'notch'. A special subclass of the notch matroids, namely the presentations where there is exactly one notch at the top and the bottom (see Figure 1.2 receives significant attention. These are exactly the lattice-path presentations of the sparse paving matroids with exactly two circuit-hyperplanes that partition the ground set. We conclude this part by proving that this subclass of notch matroids essentially forms the unique anti-chain for lattice-path matroids. This resolves a question posed by Jim Geelen. 


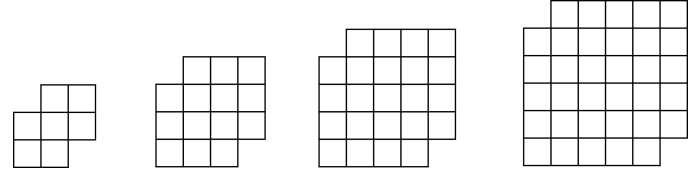

Figure 1.2: Examples of notch matroid presentations

The last part of the thesis deals with the elusive class of fundamental transversal matroids. The initial chapter deals with the complexity of recognizing the property of being fundamental in a transversal matroid and this results in the following (note that the input in both cases is a bipartite graph that corresponds to the matroid):

Theorem 1.1.8. The question of recognising fundamental transversal matroids is in NP.

Conjecture 1.1.9. The question of recognising fundamental transversal matroids is NP-complete.

The last chapters are devoted to finding algorithms that can recognise when a matroid is fundamental in the classes of bicircular and lattice-path matroids. We prove that there exist polynomial-time algorithms to recognise the same.

Theorem 1.1.10. There exists a polynomial-time algorithm to check whether a lattice-path matroid is fundamental.

Theorem 1.1.11. There exists a polynomial-time algorithm to check whether a bicircular matroid is fundamental.

We end the discussion by exploring the intersection of bicircular and cobicircular matroids. We define a class of matroids - whirly-swirls - that is both bicircular and cobicircular. We conjecture that any matroid in the intersection is eventually a whirly-swirl (that is, any large-enough matroid in this intersection is a whirly-swirl,) and close with the following result:

Theorem 1.1.12. If $G$ has a loop at every vertex and the corresponding bicircular matroid is 3-connected, bicircular and cobicircular, then $G$ is a whirly-swirl. 


\section{Chapter 2}

\section{Preliminaries}

\subsection{Transversal matroids}

Let $E$ be a set and $B=\left(N_{i}: i \in J\right)$ be an indexed family of subsets of $E$, where $J=\{1, \ldots, r\}$ and $r$ is a positive integer. Let $X=\left\{e_{1}, \ldots, e_{r}\right\}$ be a subset of $E$ where each $e_{i}$ is unique. Then $X$ is defined to be a transversal of $(E, B)$ if $e_{i} \in N_{i}$ for every $i$. Alternatively, $X$ is a transversal of $(E, B)$ if there is a bijection $\phi: J \rightarrow X$ such that $\phi(i) \in N_{i}$ for every $i \in J$. A subset $Y$ of $E$ is a partial transversal of $(E, B)$ if $Y$ is a transversal of $\left(E, B^{\prime}\right)$ where $B^{\prime}=\left(N_{i}: i \in K\right)$ for some subset $K$ of $J$. Then, the partial transversals of $(E, B)$ form the the independent sets of a matroid $M$ on the ground set $E$ [28, Ch. 1, Sec. 6]. This is defined to be a transversal matroid.

Another way to understand transversal matroids is via matchings in bipartite graphs. Let $E$ and $B$ be as defined above. Let $G(E, B)$ be a bipartite graph with with partition $(E, B)$ where $x \in E$ is adjacent to $N_{i}$ if and only if $x \in N_{i}$. (When considering bipartite graphs, it is natural for $B$ to be a multiset.) Then $X \subseteq E$ is a partial transversal of $(E, B)$ if and only if there is a matching in $G(E, B)$ in which every edge has one endpoint in $X$. Figure 2.1 shows a bipartite graph and a partial transversal in it. 

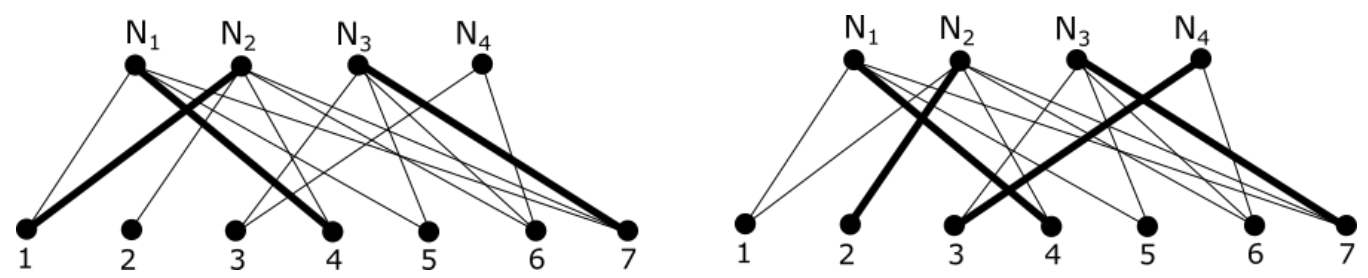

Figure 2.1: A bipartite graph with a matching that corresponds to a partial transversal on the left and a matching that corresponds to a transversal on the right. Hence, $\{1,4,7\}$ and $\{2,3,4,7\}$ are independent in the corresponding transversal matroid. Moreover $\{2,3,4,7\}$ is a basis as it is a maximum matching.

Transversal matroids have a very elegant geometric representation. A rank $r$ transversal matroid can be represented on an $r$-simplex. Cyclic flats help us understand this better.

Definition 2.1.1. A flat $F$ in a matroid is cyclic if it is a (possibly empty) union of circuits.

Then the following theorem [3] states how we can represent a transversal matroid geometrically and Figure 2.2 provides an illustration for the same:

Theorem 2.1.2. A matroid $M$ is transversal if and only if it has a geometric representation on a simplex $\Delta$ in which each cyclic flat $F$ of $M$ consists of the set of elements in some $r(F)$-vertex face of $\Delta$.
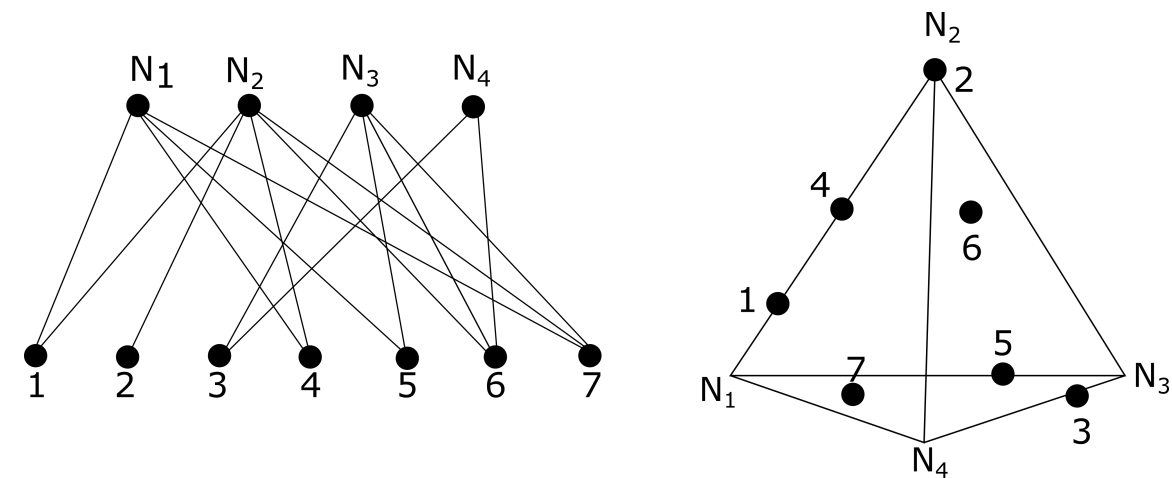

Figure 2.2: A transversal matroid $M$ represented as a bipartite graph on the left and on a simplex on the right. Each $N_{i}$ in the graph is a vertex in the simplex. If $e \in E$ is adjacent to $N_{1}, \ldots, N_{k}$ in the graph, then it is on the $k$-vertex face covered by the corresponding vertices. 


\subsubsection{Minors, duals and more}

The class of transversal matroids is not closed under contraction and hence does not form a minor-closed class. Neither is it closed under duality even though we will soon see that it has sub-classes that exhibit one or both of these properties. Figures 2.3 and 2.4 illustrate this property.

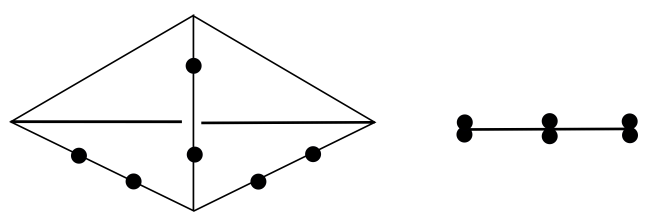

Figure 2.3: A transversal matroid $M$ on the left, and the dual on the right

Consider the matroid represented on the right in Figure 2.3. It is a rank-2 matroid with 6 elements. In the geometric representation of a transversal matroid on a simplex, a parallel pair must be placed on a vertex, otherwise known as a 0 -face, of the simplex. Since a 2 -simplex only has two vertices, this is impossible in the dual of the transversal matroid considered in Figure 2.3.

Duals of transversal matroids are called cotransversal or strict gammoids. A gammoid is any matroid that is the restriction of a strict gammoid.

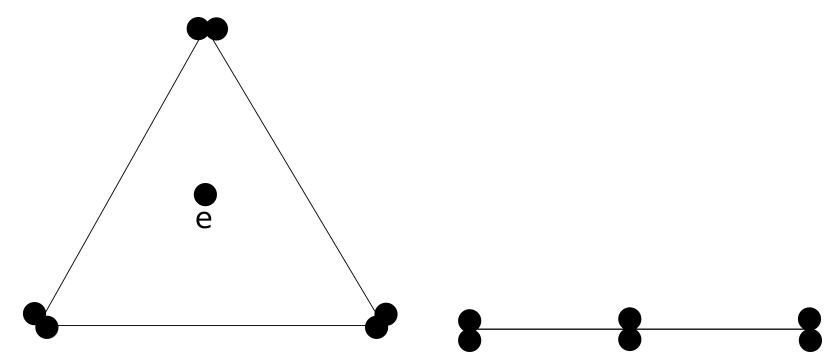

Figure 2.4: A transversal matroid $M$ on the left, and $M / e$ on the right

Tranversal matroids are closed under deletion but not under contraction. Figure 2.4 gives an example of a transversal matroid $M$, where $M / e$ is no longer transversal, the reason for which is explained above. 


\subsection{Fundamental transversal matroids}

Fundamental transversal matroids form a sub-class of transversal matroids with appealing properties. We first define a fundamental basis.

Definition 2.2.1. Let $B$ be a basis of a matroid $M$. Then, $B$ is said to be fundamental if whenever $Z$ is a cyclic flat, the intersection of $B$ and $Z$ spans Z.

Definition 2.2.2. A matroid with a fundamental basis is said to be a fundamental matroid.

We have already seen that transversal matroids can be represented by placing the elements of the matroid freely on the faces of a simplex, with all dependencies evident from the structure of the simplex. Every cyclic flat $F$ in a transversal matroid is represented on a $r(F)$-face of the simplex. Then, fundamental transversal matroids are exactly those transversal matroids that can be represented with an element on each vertex of the simplex.

In the following paragraphs, we define another notion that helps explain what it means to be a fundamental basis.

Definition 2.2.3. Let $F$ be a flat of the matroid $M$. Let e be an element in $F$. We say that $e$ is free in $F$ if: whenever $Z$ is a cyclic flat containing e, $Z$ contains $F$.

The following lemma is an elementary result that follows from the above definition and has not appeared in the literature before.

Lemma 2.2.4. $B$ is a fundamental basis of a matroid $M$ if and only if for every element e not in $B$, e is freely placed in the flat spanned by the fundamental circuit $C(e, B)$.

Proof. Let $B$ be a fundamental basis. Assume that there exists an element $e \notin B$ such that $e$ is not freely placed in the flat $F=\operatorname{cl}(C(e, B))$ spanned by the fundamental circuit $C(e, B)$. Then there exists a cyclic flat $Z$ that contains $e$ but does not contain $F$. Now $B \cap Z$ spans $Z$. So let $B^{\prime} \subseteq B$ be such that $B^{\prime}$ is a basis of $M \mid Z$. Since $B^{\prime}$ spans $e, B^{\prime} \cup e$ contains a circuit. Then, by uniqueness of the fundamental circuit, this is $C(e, B)$ 
which contradicts the fact that $Z$ does not contain $C(e, B)$. Hence, the forward direction is proved.

Now, we assume that every element $e \notin B$ is freely placed in $\operatorname{cl}(C(e, B))$. We again employ proof by contradiction and assume that $B$ is not fundamental. Then there exists a cyclic flat $Z$ such that $B \cap Z$ does not span $Z$. Hence, we can let $e$ be in $Z$ but not in $\operatorname{cl}(B \cap Z)$. Since, $e$ is freely placed in $\operatorname{cl}(C(e, B)), Z$ contains $\mathrm{cl}(C(e, B))$. But this implies that $B \cap Z$ contains $C(e, B)-e$, which in turn implies that $B \cap Z$ spans $e$, a contradiction.

Next, we prove a lemma that has, strangely enough, not appeared in the literature before.

Lemma 2.2.5. A fundamental matroid is transversal.

Proof. Let $E$ be the ground set of a matroid $M$ and let $B=\left\{b_{1}, \ldots, b_{r}\right\}$ be a fundamental basis. We construct the bipartite graph $G$ consisting of the bipartition $V \cup E$, where $V=\left\{v_{1}, \ldots, v_{r}\right\}$, as follows: Join each $b_{i}$ to $v_{i}$ by an edge. Now, consider $e \in E-B$. Let $B_{e}=\left\{b_{i_{1}}, \ldots, b_{i_{t}}\right\}$ be the subset of $B$ such that $C(e, B)=B_{e} \cup e$. Then we construct an edge between $e$ and every $v_{i_{1}}, \ldots, v_{i_{t}}$. We complete the construction of $G$ when we repeat the process for every $e \in E-B$.

Now, let $N$ be the corresponding transversal matroid that arises from $G$. We show that every rank- $k$ cyclic flat of $M$ is a rank- $k$ cyclic flat of $N$ and vice versa, as matroids are defined by cyclic flats and their ranks. Let $Z$ be a rank- $k$ cyclic flat of $M$. Then $|B \cap Z|=k$. If $e \in Z-B$ is adjacent to a $v_{i}$ that is not a neighbour of any element in $B \cap Z$, then $Z$ does not contain $c l_{M}\left(C_{M}(e, B)\right)$ (by virtue of construction of $G$ ). Since $Z$ is cyclic, there exists a circuit $C \subseteq Z$ such that $e \in C$. So $\operatorname{cl}(C) \subseteq \operatorname{cl}(Z)$ but $\operatorname{cl}(C(e, B)) \nsubseteq$ $\operatorname{cl}(C)$. Then $e$ is not free in $c_{M}\left(C_{M}(e, B)\right)$, which leads to a contradiction by Lemma 2.2.4.

Also, if $e \notin B$ is adjacent only to neighbours of $B \cap Z$, then $e$ is in $c l_{M}(B \cap Z)$ and hence $e \in Z$. This leads us to the conclusion that $Z$ consists of all elements in $Z \cap B$ and the elements in $Z-B$ are adjacent only to neighbours of $Z \cap B$. Moreover, every element in $Z \cap B$ is contained in some fundamental circuit $C(e, B)$ where $e \in Z-B$. If not, $z \in B \cap Z$ is not in any fundamental circuit. Then, $(B \cap Z)-z$ spans $Z-B$. But $z$ is 
in the span of $Z-z$, and hence it is in the span of $(B \cap Z)-z$, which is impossible. Hence, $N \mid Z$ has no coloops. Thus, there exists $H \subseteq V$ such that $H=N(Z \cap B)$, the set of neighbours of $Z \cap B$. Let $H^{c}$ be the complement of $H$ in $V$. Then, $Z$ is the complement of the neighbourhood of $H^{c}$. In other words, $Z=E-N\left(H^{c}\right)$. Observe that any rank- $k$ cyclic flat is represented on a $k$-vertex face on the simplex. So in the graph $G$, a cyclic flat is the complement of a neighbourhood of a subset of $V$. Thus, $Z$ is a cyclic flat of $N$. Since $N$ is spanned by $B \cap Z$ in $N$, it is also a rank-k cyclic flat.

Now consider a rank- $k$ cyclic flat $Z$ in $N$. We show that $Z$ is a rank- $k$ cyclic flat in $M$ too. Clearly, $c l_{N}(B \cap Z)=Z$. Let $F=c l_{M}(B \cap Z)$. If $b \in B-Z$, then $b \notin F$, as otherwise $B$ contains a circuit of $M$. Every $e \in F-B$ is in a circuit $C \subseteq(B \cap Z) \cup e$. Let $b \in B \cap Z$. Since $Z$ is a cyclic flat of $N, b$ is joined by a path of length two to some $z \in Z-B$. So $b \in C_{M}(z, B)-z \subseteq(B \cap Z)$. This implies that

$$
Z=\bigcup_{e \in Z-B} C_{N}(e, B)=\bigcup_{e \in Z-B} C_{M}(e, B)=F
$$

and hence $F$ is a cyclic flat of rank- $k$.

We have seen that the class of transversal matroids is not closed under duality. On the other hand, fundamental transversal matroids form a dual-closed class. [7]

Theorem 2.2.6. [7] The dual of a fundamental transversal matroid is a fundamental transversal matroid.

This fact was derived from the more illuminating idea that is stated below in [7]:

Lemma 2.2.7. Let $B$ be a fundamental basis in $M$, where $M$ is a matroid on ground set $E$. Then $E \backslash B$ is a fundamental basis in $M^{*}$.

Similar to transversal matroids, the class of fundamental matroids is not minor-closed, as is evident from Figure 2.4. Furthermore, this class is not closed under deletion. 
Since transversal matroids do not have unique representations as simplices or bipartite graphs, it makes it rather difficult to figure out when a given transversal matroid is fundamental. Even though they have a unique maximal bipartite presentation [3], this does nothing to reduce the complexity of this problem. This is the problem we address in Section 9.

\subsection{Lattice-path matroids}

The class of lattice-path matroids is an attractive class with nice structural properties discovered by Bonin, de Mier and Noy [6]. It is a subclass of transversal matroids, but surprisingly is closed under duality and minors, unlike the class of transversal matroids. Interestingly enough, lattice-path matroids are not well-quasi-ordered (for examples, refer to Chapter 7). However, we later prove that they are well-quasi-ordered when certain restrictions are placed on the class.

The class of lattice-path matroids can be best understood in terms of lattice-paths in the 2-dimensional integral grid. All lattice-paths considered here start at $(0,0)$ and use the steps $E=(1,0)$ (or moving right) and $N=(0,1)$ (or moving up), which are called East and North respectively. The paths are written as words or strings in the alphabet $\{E, N\}$.

In the definitions following, if $X$ is a lattice-path with $m+r$ steps, then $\operatorname{pre}_{i}(X)$ denotes the first $i$ steps of $X$ and $\operatorname{suf}_{i}(X)$ denotes steps $i+1$ to $m+r$. Hence, we can consider the path $P$ as $P=\operatorname{pre}_{i}(P) \operatorname{suf}_{i}(P)$, where juxtaposition indicates concatenation of strings. Also if $X$ is any lattice-path, then $r(X)=$ number of North steps in $X$, and $m(X)=$ number of East steps in $X$. Note that we use the notation $[n]$ to denote $\{1,2, \ldots, n\}$ and $[m, n]$ to denote $\{m, m+1, \ldots, n\}$.

Let $P=p_{1} p_{2} \ldots p_{m+r}$ and $Q=q_{1} q_{2} \ldots q_{m+r}$ be two lattice-paths from $(0,0)$ to $(m, r)$, where $P$ never goes above $Q$. In other words, for every $i$, the number of North steps in $\operatorname{pre}_{i}(P)$ is never more than that in $\operatorname{pre}_{i}(Q)$ (and the number of East steps in $\operatorname{pre}_{i}(Q)$ is never more than that in pre $\left._{i}(P)\right)$. Let $p_{u_{1}}, p_{u_{2}}, \ldots, p_{u_{r}}$ be the set of North steps of $P$, with $u_{1}<u_{2}<\cdots<u_{r}$. Let $q_{l_{1}}, q_{l_{2}}, \ldots, q_{l_{r}}$ be the set of North steps of $Q$, with $l_{1}<l_{2}<\cdots<l_{r}$. 
Note that $l_{i} \leq u_{i}$ for all $i$ by virue of definition of $P$ and $Q$. Let $N_{i}$ be the interval $\left[l_{i}, u_{i}\right]$ of integers. Let $M[P, Q]$ be the transversal matroid that has ground set $[m+r]$ and presentation $\left(N_{i}: i \in[r]\right)$. A lattice path matroid is a transversal matroid that is isomorphic to $M[P, Q]$ for some such pair of lattice-paths $P$ and $Q$.

We say that $[P, Q]$ is a lattice path presentation that corresponds to the matroid $M[P, Q]$. The size of a presentation is nothing but the size of the ground set of the corresponding matroid. We use $r$ and $m$ to denote the rank and corank of $M[P, Q]$ respectively. We blur the distinction between the path presentation $[P, Q]$ and the matroid $M[P, Q]$ when doing so will not create confusion.

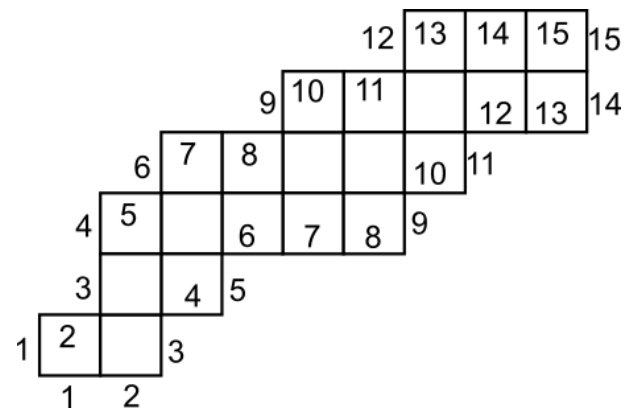

Figure 2.5: A lattice-path presentation $[P, Q]$, where the paths $P$ and $Q$ start at $(0,0)$ and end at $(9,6)$

Figure 2.5 gives an illustration of a lattice-path presentation. Here $P=$ EENENEEENENEENN and $Q=$ NENNENEENEENEEE. Also $N_{1}=$ $[1,3], N_{2}=[3,5], N_{3}=[4,9], N_{4}=[6,11], N_{5}=[9,14]$ and $N_{6}=[12,15]$.

When thought of as arising from the particular presentation of bounding paths $P$ and $Q$, the elements are in their natural order. However this order is not evident in the matroid structure.

Let $X$ be a subset of the ground set $[m+r]$ of the lattice-path matroid $M[P, Q]$. The lattice-path $P(X)$ is the word $s_{1} s_{2} \ldots s_{m+r}$ in the alphabet $\{E, N\}$, where

$$
s_{i}= \begin{cases}N, & \text { if } i \in X \\ E, & \text { otherwise. }\end{cases}
$$

This leads to the following characterisation of bases of lattice-path 
matroids:

A subset $B$ of $[m+r]$ with $|B|=r$ is a basis of $M[P, Q]$ if and only if $P(B)$ stays in the region bounded by $P$ and $Q$ (Theorem 3.3, [6]). That is, $\operatorname{pre}_{i}(P(X))$ has no more North steps than $\operatorname{pre}_{i}(Q)$ for all $i$, and no more East steps than $\operatorname{pre}_{i}(P)$ for all $i$. Note that the paths $P$ and $Q$ also stay in the desired region and hence correspond to bases. Alternatively, from the transversal definition of a lattice-path matroid, it follows that $B=\left\{b_{1}, b_{2}, \ldots, b_{r}\right\}$ is a basis of $M[P, Q]$ if $b_{i} \in N_{i}$ for all $i \in\{1, \ldots, r\}$.

\subsubsection{Minors, duals and more}

Lattice-path matroids are closed under minors, duals and direct sums (Theorem 3.1, [5]) but are not closed under the operations of truncation, elongation and free extension. The dual of a lattice-path matroid $M[P, Q]$ is $M[Q, P]$. Visually, this corresponds to finding the reflection of the presentation along the line $y=x$ as seen in Figure 2.6.
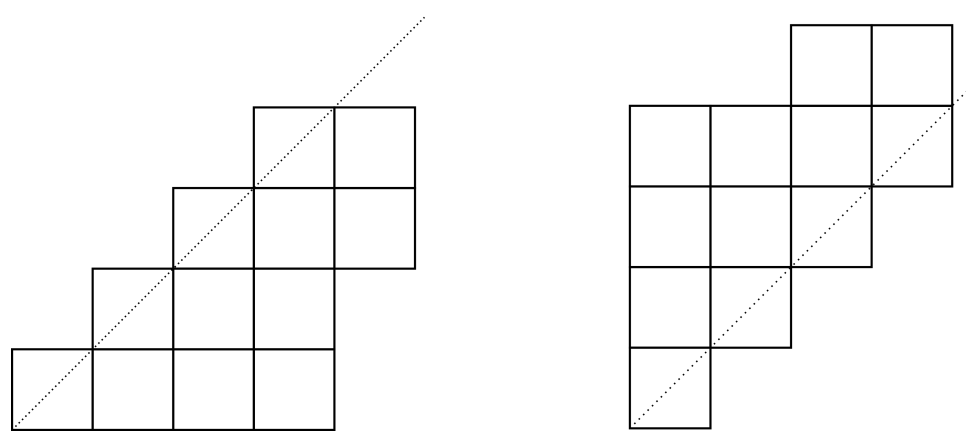

Figure 2.6: A lattice-path matroid on the left and its dual on the right

Let $[P, Q]$ be a lattice-path presentation. Single element deletions and contractions in $M=M[P, Q]$ can be described in terms of bounding paths of $[P, Q]$ as follows: A coloop is an element $x$ for which some $N_{i}$ is $\{x\}$. So, to delete or contract $x$, delete the corresponding North step from both the bounding paths. Correspondingly, to delete or contract a loop, which is an element that is in no set $N_{i}$, delete that East step from both the bounding paths.

If $x$ is neither a loop nor a coloop, the upper bounding path of $M \backslash x$ is formed by deleting from $Q$ the first East step that is at or after $x$ and the 
lower bounding path is obtained by deleting from $P$ the last East step that is at or before $x$. Dually, the upper bounding path of $M / x$ is formed by deleting from $Q$ the last North step that is at or before $x$ and the lower bounding path is obtained by deleting from $P$ the first North step that is at or after $x$.
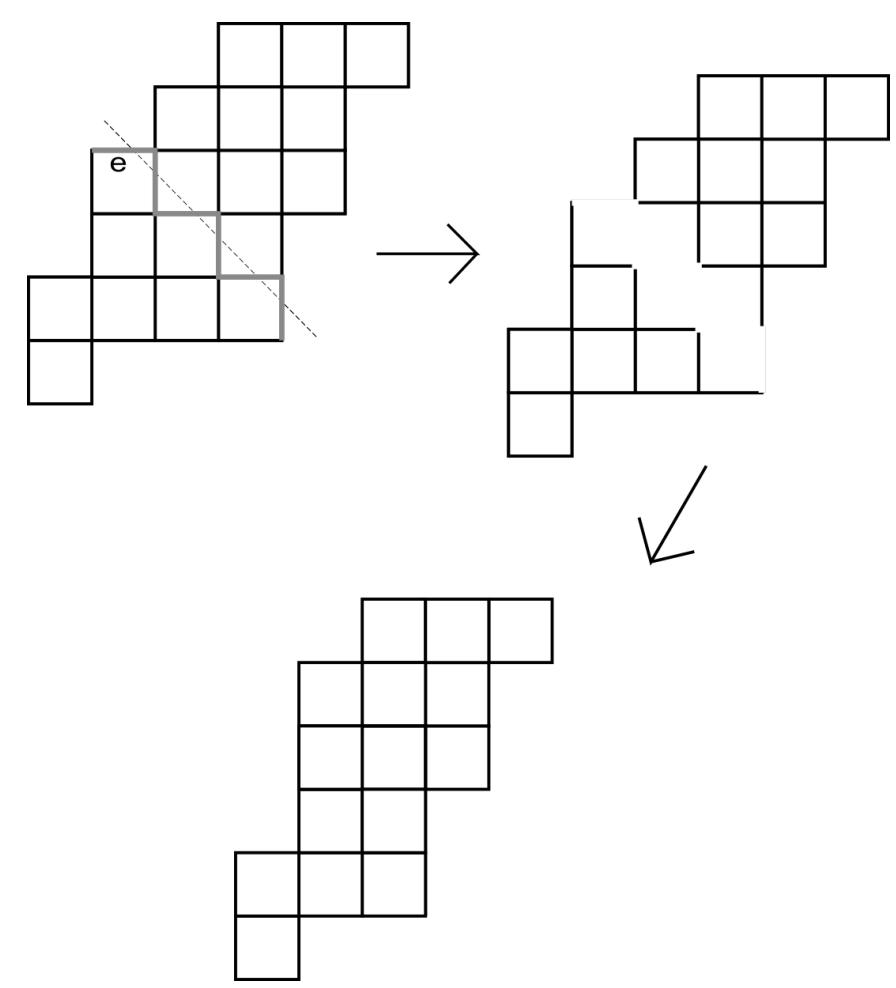

Figure 2.7: Deleting $e$ : We erase the step corresponding to $e$, then slide the remaining presentation parts horizontally and erase any bits that stick out

We define a lattice-path presentation to be a minor of another if it can be obtained by the operations of deletions and contractions, as described above, from the other.

Figures 2.7 and 2.8 shows a lattice-path presentation $[P, Q]$ with $P=$ ENEEENNENNEN and $Q=N N E N N E N E N E E E$. In Figure 2.7, we delete $e$ to obtain $P=$ ENEENNENNEN and $Q=$ NNENNNENEEE. In Figure 2.8, we contract $e$ to obtain $P=$ ENEEENENNEN and $Q=$ NNENENENEEE.

Nested matroids form a subclass of lattice-path matroids that has appeared under different names in varying contexts. A nested matroid is obtained from the empty matroid by iterating the operations of adding 

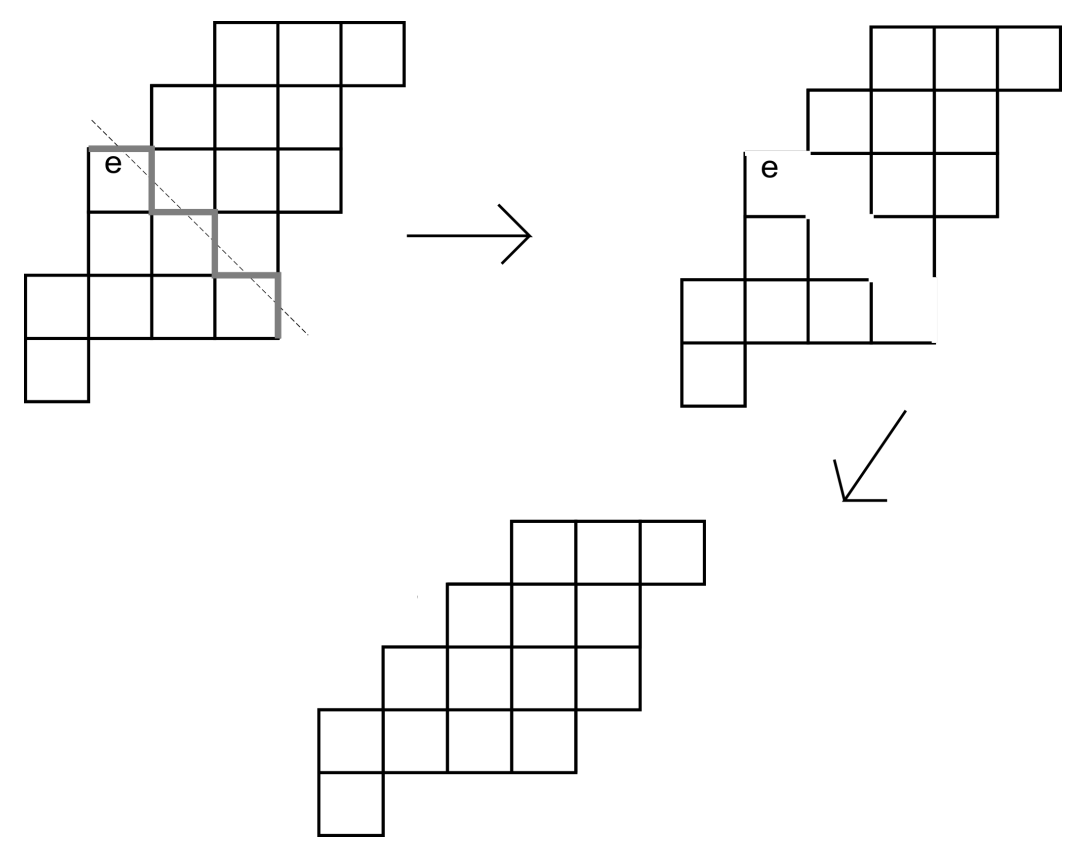

Figure 2.8: Contracting $e$ : We erase the step corresponding to $e$, then slide the remaining presentation parts vertically and erase any bits that stick out

co-loops and taking free extensions. Bonin and de Mier [5] defined them in terms of lattice-path matroids as a matroid of the form $M[P, Q]$, where $P=E^{m} N^{r}$ and named them generalised Catalan matroids. They later proved that nested matroids are well-quasi-ordered (Theorem 5.4, [4]).

\subsection{Bicircular matroids}

Bicircular matroids are a natural class of matroids that arise from graphs. Even though cycle matroids were studied from the conception of matroid theory, bicircular matroids were not introduced until four decades later by Simões Pereira [31]. Bicircular matroids can be approached in two different ways: as a matroid defined on the edge set of a graph and as a subclass of transversal matroids. We use the former approach first.

Let $G$ be a graph. Note that all graphs mentioned may have loops or parallel edges. Consider two distinct vertices $u$ and $v$. A theta subgraph is the union of three internally disjoint paths that start and end at $u$ and v. A loose handcuff is formed when two disjoint cycles are joined by a minimal path and a tight handcuff is formed when two cycles share exactly 
one vertex (see Figure 2.9). Then the matroid $B(G)$ where the set of circuits is the collection of all theta subgraphs, loose handcuffs and tight handcuffs is a matroid defined on the edge set of $G$ [31]. Equivalently, the set of independent sets is the collection of sets $X$ such that every component of $G[X]$ contains at most one cycle [22].
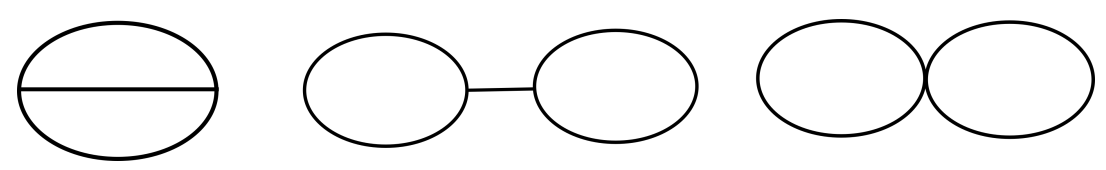

Figure 2.9: The different circuits in a bicircular matroid

Alternatively, we can also view $B(G)$ as a transversal matroid. The following theorem [22] makes it clear:

Theorem 2.4.1. Let $G$ be a graph and for each $v \in V(G)$, let $A_{v}$ be the set of edges incident with $v$. Then $B(G)$ is isomorphic to the transversal matroid $M\left(\mathcal{A}_{G}\right)$, where $\mathcal{A}_{G}=\left(A_{v}: v \in V(G)\right)$.

Correspondingly, in the simplex representation, every element will either be on a vertex or on a line.
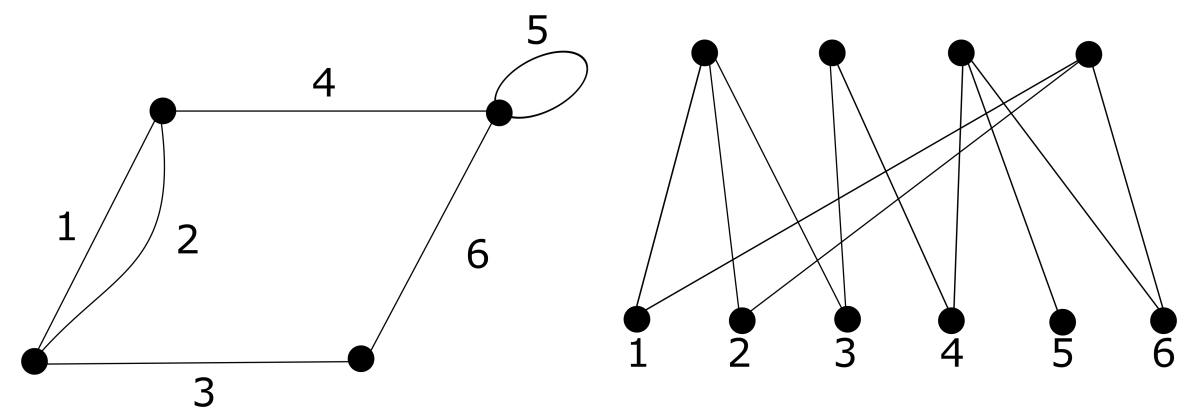

Figure 2.10: A graph on the left and the bipartite presentation of the corresponding bicircular matroid

Deleting an element $e$ in $B(G)$ is the same as deleting the corresponding edge in $G$ and finding the new bicircular matroid. In other words, $B(G) \backslash e \cong B(G \backslash e)$. The same does not hold true for contraction. Contracting a non-loop edge in $G$ is the same as contraction in graphs. But if $e$ is a loop, then we have to take a different path. Prior to that, we acknowledge that bicircular matroids do not form a minor-closed class. But the following theorem [22] shows that it does behave well to an extent: 
Theorem 2.4.2. Any loopless minor of a bicircular matroid is bicircular.

Loopless minors are obtained when we delete an element or when we contract a non-parallel element in $B(G)$. Now, we demonstrate how to contract a loop. Let $v$ be the vertex incident with the loop and $e_{1}, \ldots, e_{k}$ be the edges incident with $v$ and $v_{1}, \ldots, v_{k}$ respectively. Since we are contracting a non-parallel edge, none of $e_{1}, \ldots, e_{k}$ is a loop in $G$. Then, to contract $e$, we delete $e$ and $v$ while replacing every edge $e_{i}$ with a loop at $v_{i}$.

\subsection{Algorithms and Complexity}

A problem is in NP if whenever there is a positive answer, then there is a polynomial-time verifiable certificate that shows the answer is positive. Let $\sigma$ be a finite alphabet. Let $\sigma^{*}$ be the set of finite words. A language is a subset of $\sigma^{*}$. Let $L$ be a language. We say $L$ is in $P$ if there is a Turing machine, running in time that is polynomially bounded by $|w|(|w|$ is the the length of the word), which will take any word in $\sigma^{*}$ as input, and accept it if and only if it is in $L$. Say that $R$ is a relation from $\sigma^{*}$ to $\sigma^{*}$ such that whenever $\left(w, w^{\prime}\right)$ is in $R, w$ is in $L$. Also, whenever $w$ is in $L$, there exists $w^{\prime}$ such that $\left(w, w^{\prime}\right)$ is in $R$ and there is a polynomial-time Turing machine which will take any pair $\left(w, w^{\prime}\right)$ as input, and decide if it is in $R$. Then we say $L$ is in NP. We think of $w^{\prime}$ as being a certificate that $w$ is in $L$.

For example consider the problem HAMILTONIAN CYCLE described as follows:

INPUT: A graph G

QUESTION: Is G Hamiltonian?

To prove that the problem is HAMILTONIAN CYCLE in NP, one has to show that there exists a Turing machine $T$ that works in polynomial-time such that given a graph $G$ and a $y, T$ accepts if and only if $y$ "witnesses" that $G$ has a Hamiltonian cycle. The machine $T(G, y)$ does the following: it assumes that $y$ is a sequence of nodes in $G$. For each $v_{i}, v_{i+1}$ in this 
sequence, $T$ checks that there is an edge from $v_{i}$ to $v_{i+1}$ in $G$. If this is the case for all $i$ and if the last vertex is equal to the first one, and if the vertices of $G$ are in the list, $T(G, y)$ accepts, and otherwise it rejects.

In the discussions following, we prove that the question of deciding when a transversal matroid is fundamental is in NP and conjecture that is NP-complete. 


\section{Part I}

\section{Definability and Recognisability}



In this part, we start by describing monadic second-order logic and developing atomic formulas, abbreviations and rewriting definitions that are relevant to the chapters that follow.

In Chapter 4, we further develop monadic second-order logic to equip it with machinery required to axiomatise lattice-path and fundamental matroids. We then successfully achieve the same in the said chapter. We end with a conjecture on the definability of transversal matroids in $M S_{0}^{+}$. Finally in Chapter 5, we comment on the existing literature that disproves recognisability for the classes of transversal, fundamental and lattice-path matroids and provide an alternate proof that there exists no polynomial-time algorithm to test whether a given matroid is bicircular. The concept of an independence oracle is explored along with its significance in proving these results. 


\section{Chapter 3}

\section{Monadic second-order Logic}

Whitney's axioms for matroids are written in a logical language that quantifies over subsets of the ground set. For example, we say for every subset $I$ and $I^{\prime}$, if $I^{\prime} \subseteq I$ and $I$ is an independent set, then $I^{\prime}$ is independent. In this section we make such a language formal and consider its expressive power. The language we describe here is called monadic second order logic and is almost the same as the language used in [19] and [26].

A property is said to be definable in a language if there is a sentence that can define the said property. Representable matroids are not definable in monadic second order logic [25] but families of matroids with finitely many excluded minors can be defined. That said, even though the class of lattice path matroids has infinitely many excluded minors, we prove in Chapter 4 that they can be defined in $M S_{0}$ with the rank function (which we denote by $\mathrm{MS}_{0}^{+}$henceforth). We further conjecture that it is definable in $M S_{0}$. Later, in Chapter 4 , we prove that fundamental matroids are also definable in $M S_{0}^{+}$.

$\mathrm{MS}_{0}^{+}$for matroids is a formal language constructed from the following symbols:

1. Variables $X_{1}, X_{2}, X_{3}, \ldots$

2. Unary predicates Ind and Sing

3. Binary predicates $\subseteq$ and $\leq$ 
4. Connectives $\neg$ and $\wedge$

5. Quantifier $\exists$

6. Function symbols $r(X),+$ and $|\cdot|$

We will think of $\operatorname{Ind}(X)$ as being true when $X$ is independent and $\operatorname{Sing}(X)$ as being true when $X$ is a singleton set.

Now we construct the language $M S_{0}^{+}$recursively.

Terms: Let $\mathcal{T}$ be the set of terms. We define the elements of this set, or in other words, the terms in $\mathcal{T}$, and the corresponding set of variables. If $T$ is a term, then $\operatorname{Var}(T)$ stands for the set of variables in that term.

1. For every variable $X, r(X)$ and $|X|$ are terms and

$$
\operatorname{Var}(r(X))=\operatorname{Var}(|X|)=\{X\} .
$$

2. For every term $T$, we have that $T+r(X)$ and $T+|X|$ are also terms and

$$
\operatorname{Var}(T+r(X))=\operatorname{Var}(T+|X|)=\operatorname{Var}(T) \cup\{X\} .
$$

Formulas: Now we recursively define formulas in $M S_{0}^{+}$, and simultaneously define their sets of variables. If $\phi$ is a formula, then $\operatorname{Var}(\phi)$ is the set of variables of the formula and $\operatorname{Fr}(\phi)$ is the set of free variables as is going to be defined recursively below. The following four statements define expressions known as atomic formulas.

1. If $X$ is a variable, then $\operatorname{Sing}(X)$ is an atomic formula and

$$
\operatorname{Var}(\operatorname{Sing}(X))=\operatorname{Fr}(\operatorname{Sing}(X))=\{X\} .
$$

2. If $X$ is a variable, then $\operatorname{Ind}(X)$ is an atomic formula and

$$
\operatorname{Var}(\operatorname{Ind}(X))=\operatorname{Fr}(\operatorname{Ind}(X))=\{X\} .
$$


3. If $X$ and $Y$ are variables, then $X \subseteq Y$ is an atomic formula and

$$
\operatorname{Var}(X \subseteq Y)=\operatorname{Fr}(X \subseteq Y)=\{X, Y\}
$$

4. If $T_{1}$ and $T_{2}$ are terms, then $T_{1} \leq T_{2}$ is an atomic formula and

$$
\operatorname{Var}\left(T_{1} \leq T_{2}\right)=\operatorname{Fr}\left(T_{1} \leq T_{2}\right)=\operatorname{Var}\left(T_{1}\right) \cup \operatorname{Var}\left(T_{2}\right)
$$

We now define the non-atomic formulas. Let $\phi_{1}$ and $\phi_{2}$ be formulas.

1. $\neg\left(\phi_{1}\right)$ is a formula where $\operatorname{Var}\left(\neg \phi_{1}\right)=\operatorname{Var}\left(\phi_{1}\right)$ and $\operatorname{Fr}\left(\neg \phi_{1}\right)=\operatorname{Fr}\left(\phi_{1}\right)$.

2. If

$$
\operatorname{Fr}\left(\phi_{1}\right) \cap\left(\operatorname{Var}\left(\phi_{2}\right)-\operatorname{Fr}\left(\phi_{2}\right)\right)=\operatorname{Fr}\left(\phi_{2}\right) \cap\left(\operatorname{Var}\left(\phi_{1}\right)-\operatorname{Fr}\left(\phi_{1}\right)\right)=\varnothing,
$$

then $\phi_{1} \wedge \phi_{2}$ is a formula with

$$
\operatorname{Var}\left(\phi_{1} \cap \phi_{2}\right)=\operatorname{Var}\left(\phi_{1}\right) \cup \operatorname{Var}\left(\phi_{2}\right) \text { and } \operatorname{Fr}\left(\phi_{1} \cap \phi_{2}\right)=\operatorname{Fr}\left(\phi_{1}\right) \cup \operatorname{Fr}\left(\phi_{2}\right) \text {. }
$$

3. If $X \in \operatorname{Fr}\left(\phi_{1}\right)$, then $\exists \phi_{1}$ is a formula where $\operatorname{Var}\left(\exists \phi_{1}\right)=\operatorname{Var}\left(\phi_{1}\right)$ and $\operatorname{Fr}\left(\exists \phi_{1}\right)=\operatorname{Fr}\left(\phi_{1}\right)-\{X\}$.

A sentence is a formula with no free variables. We often omit brackets where doing so will not create confusions.

Definition 3.1.1. $M S_{0}$ is the language constructed as above without any atomic formula of the form $T_{1} \leq T_{2}$.

Thus $M S_{0}$ is the same language as the language used in [26].

Interpretations: A structure is a triple $(E, \mathcal{I}, \sigma)$, where $E$ is a finite set, $\mathcal{I}$ is a subset of the power set of $E$ and $\sigma$ is a function such that $\sigma: 2^{E} \rightarrow \mathbb{R}$.

Let $\phi$ be a formula and $M=(E, \mathcal{I}, \sigma)$ be a structure. An interpretation of $\phi$ in $M$ is a function $\theta: \operatorname{Fr}(\phi) \rightarrow 2^{E}$. When $T$ is a term, we recursively define $\theta(T)$ as follows:

1. If $T$ is $r(X)$, then $\theta(T)=\sigma(\theta(X))$. 
2. If $T$ is $|X|$, then $\theta(T)=|\theta(X)|$.

3. If $T$ is $T^{\prime}+r(X)$, then $\theta(T)=\theta\left(T^{\prime}\right)+\sigma(\theta(X))$.

4. If $T$ is $T^{\prime}+|X|$, then $\theta(T)=\theta\left(T^{\prime}\right)+|\theta(X)|$.

We define what it means for $M$ to satisfy $\phi$ (under $\theta$ ). If $\phi$ is an atomic formula, then the following holds:

1. If $\phi$ is $\operatorname{Sing}(X)$, then $M$ satisfies $\phi$ if $|\theta(X)|=1$.

2. If $\phi$ is $\operatorname{Ind}(X)$, then $M$ satisfies $\phi$ if $\theta(X) \in \mathcal{I}$.

3. If $\phi$ is $X \subseteq Y$, then $M$ satisfies $\phi$ if $\theta(X) \subseteq \theta(Y)$.

4. If $\phi$ is $T_{1} \leq T_{2}$, then $M$ satisfies $\phi$ if $\theta\left(T_{1}\right) \leq \theta\left(T_{2}\right)$.

Now we assume that $\phi$ is non-atomic. We define satisfiability as follows:

1. If $\phi=\neg \psi$, then $M$ satisfies $\phi$ under $\theta$ if it does not satisfy $\psi$ under $\theta$

2. If $\phi=\psi_{1} \wedge \psi_{2}$, then $M$ satisfies $\phi$ under $\theta$ if it satisfies $\psi_{1}$ under the restriction $\left.\theta\right|_{\operatorname{Fr}\left(\psi_{1}\right)}$ and it satisfies $\psi_{2}$ under $\left.\theta\right|_{\operatorname{Fr}\left(\psi_{2}\right)}$.

3. If $\phi=\exists X \psi$, then $M$ satisfies $\phi$ under $\theta$ if there is some $Y \subseteq E$ such that $M$ satisfies $\psi$ under the function $\theta \cup\{(X, Y)\}$, where we consider any function to be a set of ordered pairs.

Abbreviations: For brevity, we make frequent use of the following abbreviations:

1. We use $\phi_{1} \vee \phi_{2}$ to denote $\neg\left(\neg \phi_{1} \wedge \neg \phi_{2}\right)$

2. We use $\phi_{1} \rightarrow \phi_{2}$ to denote $\neg \phi_{1} \vee \phi_{2}$

3. We use $\phi_{1} \Longleftrightarrow \phi_{2}$ to denote $\left(\phi_{1} \rightarrow \phi_{2}\right) \wedge\left(\phi_{2} \rightarrow \phi_{1}\right)$

4. We use $\forall \phi_{1}$ to denote $\neg \exists X\left(\neg \phi_{1}\right)$

5. We use $X \nsubseteq Y$ to denote $\neg(X \subseteq Y)$

6. We use $X=Y$ to denote $(X \subseteq Y) \wedge(Y \subseteq X)$ 
7. We use $X \neq Y$ to denote $\neg((X \subseteq Y) \wedge(Y \subseteq X))$

8. We use $T_{1}=T_{2}$ to denote $T_{1} \leq T_{2} \wedge T_{2} \leq T_{1}$

9. We use $T_{1}<T_{2}$ to denote $T_{1} \leq T_{2} \wedge \neg\left(T_{1}=T_{2}\right)$

\section{Rewriting definitions in $M S_{0}^{+}$:}

We reuse certain formulas enough times that it is useful to have shortcuts to refer to them.

- $\operatorname{Uni}(X, Y, Z)=\forall W \operatorname{Sing}(W) \rightarrow(W \subseteq Z \Longleftrightarrow(W \subseteq X \vee W \subseteq Y))$ is satisfied by an interpretation that takes $Z$ to the union of $X$ and $Y$.

- $\operatorname{Int}(X, Y, Z)=\forall W \operatorname{Sing}(W) \rightarrow(W \subseteq Z \Longleftrightarrow(W \subseteq X \wedge W \subseteq Y))$ is satisfied by an interpretation that takes $Z$ to the intersection of $X$ and $Y$.

- $\operatorname{GroundSet}(X)=\forall Y(\operatorname{Sing}(Y) \rightarrow Y \subseteq X)$ is satisfied when $X$ is the entire set $E$.

- $\operatorname{Bas}(B)=\operatorname{Ind}(B) \wedge \forall B^{\prime}\left(B \subseteq B^{\prime} \rightarrow\left(B=B^{\prime} \vee \neg \operatorname{Ind}\left(B^{\prime}\right)\right)\right)$ is satisfied if $B$ is interpreted as a maximal subset of $E$ with respect to being in $I$.

- $\operatorname{Bas}(B, X)=B \subseteq X \wedge \operatorname{Ind}(B) \wedge \forall B^{\prime}\left(\left(B \subseteq B^{\prime} \wedge B^{\prime} \subseteq X\right) \rightarrow(B=\right.$ $\left.\left.B^{\prime} \vee \neg \operatorname{Ind}\left(B^{\prime}\right)\right)\right)$ is satisfied if $B$ is interpreted as a maximal subset of $X$ with respect to being in $I$.

We define $M=(E, \mathcal{I}, \sigma)$ to be legitimate if it satisfies the sentence

$$
\forall X \forall B(\operatorname{Bas}(B, X) \rightarrow|B|=\sigma(X))
$$

Consider the axioms below:

(i) $\exists X \operatorname{Ind}(X)$

(ii) $\forall X_{1} X_{2}\left(\left(\operatorname{Ind}\left(X_{1}\right) \wedge X_{2} \subseteq X_{1}\right) \rightarrow \operatorname{Ind}\left(X_{2}\right)\right)$

(iii) $\forall X_{1} X_{2}\left(\left(\operatorname{Ind}\left(X_{1}\right) \wedge \operatorname{Ind}\left(X_{2}\right) \wedge\left|X_{1}\right|<\left|X_{2}\right|\right) \rightarrow(\exists X(\operatorname{Sing}(X) \wedge X \nsubseteq\right.$ $\left.\left.\left.\left.X_{1} \wedge X \subseteq X_{2} \wedge \forall X^{\prime}\left(\operatorname{Uni}\left(X_{1}, X, X^{\prime}\right) \rightarrow \operatorname{Ind}\left(X^{\prime}\right)\right)\right)\right)\right)\right)$ 
If $M=(E, \mathcal{I}, \sigma)$ is a legitimate structure satisfying the above axioms, then $(E, \mathcal{I})$ is a matroid and $\sigma$ is the rank function of this matroid. The following result was proved recently:

Theorem 3.1.2. [19] Any minor-closed class with finitely many excluded minors can be characterised by a sentence in $M S_{0}$.

Since Rota's conjecture is now being proved to be true, combining it with the above result, we can now define finite-field matroids in this language. By way of contrast, there is no sentence that can define the class of matroids that are representable over at least one field [25]. Similarly, for an infinite field, there is no sentence that defines the matroids representable over that field [25]. 


\section{Chapter 4}

\section{Definability in classes of transversal matroids}

In this chapter, we use $M S_{0}^{+}$to define lattice path matroids and fundamental matroids. Recent work on bicircular matroids has proved that they are definable in $M S_{0}$ [Private communication with Funk, Mayhew and Newman].

\subsection{Defining lattice-path matroids}

We continue rewriting definitions that are used in the context of matroids, especially ones that will help with defining lattice path matroids in this language:

1. $\operatorname{Cir}(C)=\neg \operatorname{Ind}(C) \wedge \forall C^{\prime}\left(C^{\prime} \subseteq C \rightarrow\left(C=C^{\prime} \vee \operatorname{Ind}\left(C^{\prime}\right)\right)\right)$ is satisfied when $C$ is a circuit.

2. $\operatorname{Flat}(F)=\forall B(\operatorname{Bas}(B, F) \rightarrow(\forall W \operatorname{Sing}(W) \wedge W \nsubseteq F \rightarrow$

$$
(\forall X \operatorname{Xni}(B, W, X) \rightarrow \operatorname{Ind}(X))))
$$

is satisfied if $F$ is a flat. Note that $F$ is a flat if and only if for every basis $B$ of $F$ and for every element $e$ not in $F, B \cup e$ is in $\mathcal{I}$. 
3. $\operatorname{Part}(Y, Z, X)=\forall W((\operatorname{Sing}(W) \wedge W \subseteq X) \rightarrow(W \subseteq Y \vee W \subseteq Z)) \wedge$

$$
\begin{gathered}
((\operatorname{Sing}(W) \wedge W \subseteq Y) \rightarrow(W \subseteq X \wedge W \nsubseteq Z)) \wedge \\
((\operatorname{Sing}(W) \wedge W \subseteq Z) \rightarrow(W \subseteq X \wedge W \nsubseteq Y))) \wedge \\
\exists U(\operatorname{Sing}(U) \wedge U \subseteq Y) \wedge \exists V(\operatorname{Sing}(V) \wedge V \subseteq Z)
\end{gathered}
$$

is satisfied by an interpretation that takes $Y$ and $Z$ to sets that partition $X$. Note that $Y, Z$ partition $X$ if and only for every element $x$ in $X$ is exactly in one of $Y$ or $Z$.

4. $\operatorname{Conn}(X)=\forall Y Z\left(\operatorname{Part}(Y, Z, X) \rightarrow \exists C\left(\operatorname{Circuit}(C) \wedge \exists C_{1} C_{2}\left(\operatorname{Sing}\left(C_{1}\right) \wedge\right.\right.\right.$

$$
\left.\left.\operatorname{Sing}\left(C_{2}\right) \wedge\left(C_{1} \subseteq C \wedge C_{1} \subseteq Y\right) \wedge\left(C_{2} \subseteq C \wedge C_{2} \subseteq Z\right)\right)\right)
$$

is satisfied when $X$ is connected. We use the fact that $X$ is connected if and only if for every bipartition $Y, Z$ of $X$, there is a circuit that has non-empty intersection with both $Y$ and $Z$. Thus the restriction to $X$ is a connected matroid.

5. SpanCirc(C) $=\operatorname{Circ}(C) \wedge \exists B(B \subseteq C \wedge \operatorname{Bas}(B))$ is satisfied by an interpretation that takes $C$ to a spanning circuit.

6. $\operatorname{Span}(Z, X)=Z \subseteq X \wedge \exists B(B \subseteq Z \wedge \operatorname{Bas}(B, X))$ is satisfied by an interpretation that takes $Z$ to a spanning set of $X$.

7. Fund $(F)=\operatorname{Conn}(F) \wedge$ Flat $(F) \wedge(\exists X \exists Y \operatorname{Sing}(X) \wedge \operatorname{Sing}(Y) \wedge$

$$
\begin{array}{r}
X \neq Y \wedge X \subseteq F \wedge Y \subseteq F) \wedge(\neg \exists B \operatorname{Bas}(B) \wedge B \subseteq F) \wedge \\
\quad \exists C(\operatorname{SpanCir}(C) \wedge \forall Z(\operatorname{Int}(F, C, Z) \rightarrow B a s(Z, F)))
\end{array}
$$

is satisfied when $F$ is interpreted as follows: $F$ is a connected flat that has at least two elements such that it does not contain a basis and there is a spanning circuit $C$ such that $F \cap C$ is a basis of $F$.

8. $\operatorname{Conn} \operatorname{Comp}(X)=\operatorname{Conn}(X) \wedge(\forall Y(X \subseteq Y \rightarrow X=Y \vee \neg \operatorname{Conn}(Y)))$ is satisfied when $X$ is a connected component. Note that this holds true if and only if $X$ is connected and every proper superset is not connected.

A flat satisfying the condition stated in 7 above is defined to be a fundamental flat.

If $N$ is a matroid, then there is a sentence in $M S_{0}$ which is satisfied exactly by the matroids with $N$ as a minor [19]. It was noted at the end of Chapter 
3 that if $F$ is an infinite field, then there is no sentence in $M S_{0}$ that can characterise the class of $F$-representable matroids.

On the other hand, nested matroids can be characterised even though the class has infinitely many excluded minors. In order to prove such a characterisation exists, we need the following result from [4]:

Theorem 4.2.1. A matroid is nested if and only if the lattice of cyclic flats form a chain.

We make use of the following formula to rewrite the above statement in $M S_{0}^{+}$:

$\operatorname{CycFlat}(Z)=\operatorname{Flat}(Z) \wedge \forall W((\operatorname{Sing}(W) \wedge W \subseteq F) \rightarrow \exists C(\operatorname{Circ}(C) \wedge W \subseteq$ $C \subseteq Z)$.

Thus rewriting Theorem 4.2.1, we obtain the following:

$$
\forall Z Z^{\prime}\left(\operatorname{CycFlat}(Z) \wedge \operatorname{CycFlat}\left(Z^{\prime}\right) \rightarrow\left(Z \subseteq Z^{\prime} \vee Z^{\prime} \subseteq Z\right)\right)
$$

We are now in shape to characterise the class of lattice path matroids in $M S_{0}^{+}$. We refer to [5] in order to achieve this.

Recall that we denote the co-rank of a matroid with $m$. We use $\eta$ to denote the nullity $|X|-r(X)$ of a flat. Note that the initial segment $[h]$ of $[m+$ $r]$ is a proper nontrivial connected flat, and hence a fundamental flat, if and only if the upper path $Q$ has an $E N$ corner at $h$ [5] in a lattice-path presentation $[P, Q]$. Similarly, the final segment $[k, m+r]$ of $[m+r]$ is a fundamental flat of $M[P, Q]$ if and only if $P$ has a $N E$ corner at $k-1$. The flats that are initial segments form a chain, say $F_{1} \subseteq F_{2} \subseteq \cdots \subseteq F_{h}$, and the final segments form another chain, say $G_{1} \subseteq G_{2} \subseteq \cdots \subseteq G_{k}$.

Theorem 4.2.2. [5] A connected matroid $M$ is a lattice path matroid if and only if the fundamental flats form at most two disjoint chains under inclusion, say $F_{1} \subseteq F_{2} \subseteq \cdots \subseteq F_{h}$ and $G_{1} \subseteq G_{2} \subseteq \cdots \subseteq G_{k}$, and the following properties hold:

(i) If $F_{i} \cap G_{j} \neq \phi$, then $F_{i} \cup G_{j}=E(M)$.

(ii) The proper nontrivial connected flats of $M$ are precisely the following:

(a) $F_{1}, F_{2}, \ldots, F_{h}, G_{1}, G_{2}, \ldots, G_{k}$, and 
(b) intersections $F_{i} \cap G_{j}$ for which the inequality $m<\eta\left(F_{i}\right)+\eta\left(G_{j}\right)$ holds.

(iii) The rank of the flat $F_{i} \cap G_{j}$ of item (ii:b) is $r\left(F_{i}\right)+r\left(G_{j}\right)-r(M)$

It is only necessary to characterise connected lattice path matroids as we can characterize when $X$ is a connected component and the class of lattice path matroids is closed under direct sums.

We are now in shape to characterise lattice path matroids in $M S_{0}^{+}$.

Theorem 4.2.3. Lattice-path matroids are axiomatisable in $\mathrm{MS}_{0}^{+}$.

Proof. Rewriting the Theorem 4.2.2 in $M S_{0}^{+}$, we obtain the following: (Please note that the first two conditions in the following characterisation are in fact one sentence as they are both in the scope of $\exists W_{t} W_{b}$. The sentence has been broken apart for better understanding of each condition separately.)

1. $\exists W_{t} W_{b}\left(\operatorname{Sing}\left(W_{t}\right) \wedge \operatorname{Sing}\left(W_{b}\right) \wedge\left(W_{t} \neq W_{b}\right) \wedge \forall F F u n d(F) \rightarrow\left(\left(W_{t} \subseteq\right.\right.\right.$ $\left.\left.F \wedge W_{b} \nsubseteq F\right) \vee\left(W_{b} \subseteq F \wedge W_{t} \nsubseteq F\right)\right) \wedge \forall F F^{\prime}\left(\right.$ Fund $(F) \wedge F$ Fund $\left.\left(F^{\prime}\right)\right) \rightarrow$ $\left(\left(W_{t} \subseteq F \wedge W_{t} \subseteq F^{\prime}\right) \rightarrow\left(F \subseteq F^{\prime} \vee F^{\prime} \subseteq F\right)\right) \wedge\left(\left(W_{b} \subseteq F \wedge W_{b} \subseteq\right.\right.$ $\left.\left.F^{\prime}\right) \rightarrow\left(F \subseteq F^{\prime} \vee F^{\prime} \subseteq F\right)\right)$

Here we use the top and bottom elements of the lattice path matroid to differentiate between the two chains of fundamental flats. As the matroid itself is not a fundamental flat, every fundamental flat in one chain contains the top element but not the bottom element and every fundamental flat in the other chain contains the bottom element but not the top one.

2. $\forall F F^{\prime}$ Fund $(F) F u n d\left(F^{\prime}\right)\left(\exists X\left(\operatorname{Sing}(X) \wedge X \subseteq F \wedge X \subseteq F^{\prime}\right) \wedge W_{t} \subseteq\right.$ $\left.\left.F \wedge W_{t} \nsubseteq F^{\prime} \wedge W_{b} \subseteq F^{\prime} \wedge W_{b} \nsubseteq F\right) \rightarrow \forall Z \operatorname{Sing}(Z)\left(Z \subseteq F \vee Z \subseteq F^{\prime}\right)\right)$

Here we make use of two elements to ensure that we have one fundamental flat from each chain and use a third element to show that they have non empty intersection. We then illustrate that the union is the ground set. 
3. $\forall F\left((\operatorname{Conn}(F) \wedge F \operatorname{Flat}(F)) \rightarrow \quad\left(F u n d(F) \vee \exists F_{1} F_{2}\left(F u n d\left(F_{1}\right) \wedge\right.\right.\right.$ $\operatorname{Fund}\left(F_{2}\right) \wedge \operatorname{Int}\left(F_{1}, F_{2}, F\right) \wedge\left(\forall E G r o u n d S e t(E) \rightarrow|E|+r\left(F_{1}\right)+\right.$ $\left.\left.\left.\left.r\left(F_{2}\right)<\left|F_{1}\right|+\left|F_{2}\right|\right) \wedge\left(\forall B\left(B a s(B) \wedge r(F)+|B|=r\left(F_{1}\right)+r\left(F_{2}\right)\right)\right)\right)\right)\right)$

Here we state that every connected flat is either a fundamental flat or a flat that obeys a certain rank inequality condition. We also state the rank of such flats.

We conclude this section stating the conjecture mentioned in the introduction:

Conjecture 4.2.4. Lattice path matroids are axiomatisable in $M S_{0}$.

The current literature on lattice path matroids may not be sufficient to prove this conjecture right. But we have faith in the rich structural properties exhibited by this subclass of transversal matroids.

\subsection{Defining fundamental matroids}

We continue to work towards defining fundamental matroids. By Lemma 2.2.4. we know that $B$ is a fundamental basis of matroid $M$ if and only if for every $e$ not in $B, e$ is free in its fundamental circuit with respect to $B$. In other words, $M$ is fundamental if and only if there exists a basis $B$ such that for every $e$ not in $B$, every circuit $C$, and every cyclic flat $Z$, if $C$ contains $e$ and is contained in $B \cup e$, and $Z$ contains $e$, then $Z$ contains $C$.

We first define the term required to denote a cyclic flat and then use $M S_{0}$ to rewrite the above definition of a fundamental matroid.

$\operatorname{CycFlat}(Z)=\operatorname{Flat}(Z) \wedge(\forall X \operatorname{Sing}(X) \rightarrow(X \subseteq Z \rightarrow \exists C(\operatorname{Circ}(C) \wedge X \subseteq$ $C \wedge C \subseteq Z)$ is satisfied by an interpretation that takes $Z$ to a cyclic flat.

Now, we are ready to define a fundamental matroid in $M S_{0}$.

Theorem 4.3.1. Fundamental matroids are axiomatisable in $M S_{0}$. 
Proof. A matroid $M$ is fundamental if and only if it satisfies the following condition: $\exists \operatorname{BBas}(B) \forall e \operatorname{Sing}(e)(e \nsubseteq B \rightarrow(\forall C C i r(C) \rightarrow$ $\left.\left.\left(\forall C y c F l a t(Z)\left(\left(e \in C \wedge \forall U n i\left(B, e, B^{\prime}\right) C \subseteq B^{\prime} \wedge e \in Z\right) \rightarrow(Z \subseteq C)\right)\right)\right)\right)$

The famous Mason-Ingleton theorem (stated below, as cited in [3]) is a rank inequality characteristaion for transversal matroids.

Theorem 4.3.2. A matroid $M$ is transversal if and only if for all nonempty $\mathcal{F} \subseteq$ $Z(M)$,

$$
r(\cap \mathcal{F}) \leq \sum_{\mathcal{F}^{\prime} \subseteq \mathcal{F}}(-1)^{\left|\mathcal{F}^{\prime}\right|+1} r\left(\cup \mathcal{F}^{\prime}\right)
$$

But this cannot be expressed in monadic second order logic even with the rank function as it would involve considering all the different families of flats in any given matroid. This leads us to the following conjecture:

Conjecture 4.3.3. Transversal matroids are not definable in $M S_{0}^{+}$. 


\section{Chapter 5}

\section{Oracle algorithms}

An oracle is a subroutine that an algorithm can access in any single step to solve decision problems. These decision problems can be of any complexity. The most common oracle that matroid algorithms use is an independence oracle. In an independence oracle, we imagine that we have a subroutine INDEPENDENCE[X], which we can use to query the independence of the set $X$. This makes life easier as we do not have to be concerned with how we input our matroid. Some well-studied classes of examples have efficient ways in which we can achieve this, but this cannot be said of a general matroid. Thus making use of oracles avoids inputs that consist of long lists of bases or circuits, for example.

In this section, we ask whether there is a program which can solve matroid recognisability problems in polynomial time. Imagine we have a matroid property, and we can find pairs of matroids $\left(M_{1}, N_{1}\right),\left(M_{2}, N_{2}\right), \ldots$ such that each $M_{i}$ has the property we investigate, and each $N_{i}$ does not and furthermore deciding whether the matroid described by the oracle is $M_{i}$ or $N_{i}$ takes more than a polynomial number of calls to the oracle. Then, there cannot be a polynomial-time algorithm for testing said property. This is the approach employed by Jensen and Korte [20]. By the results in that paper, there is no polynomial-time oracle algorithm for testing whether a matroid is transversal or fundamental transversal. Bonin et al. [5] claim that the same result applies to the class of lattice path matroids. Chen and Whittle [8] prove that this is true for frame matroids. Since the proof employs bicircular matroids, the proof can also imply 
that there is no polynomial time algorithm for testing the property of being a bicircular matroid. We arrive at the same conclusion via the aforementioned technique of Jensen and Korte.

Theorem 5.1.1. There exists no polynomial-time algorithm to test whether a given matroid is bicircular.

Proof. We claim that there are two classes of matroids, $\mathcal{M}$ and $\mathcal{N}$, where $\mathcal{M}$ consists solely of bicircular matroids and $\mathcal{N}$ does not, such that no polynomial time algorithm can differentiate between both. Let $\mathcal{M}$ be the class of all bicircular matroids $B\left(G_{r}\right)$, where $G_{r}$ is a graph that is a cycle of size $r$ with every edge replaced by a parallel pair and a loop at every vertex. Now, we consider $\mathcal{N}$ as the class of all matroids $N_{r}$ such that every $B\left(G_{r}\right)$ is a relaxation of $N_{r}$, that is a basis of $B\left(G_{r}\right)$ is now a circuit hyperplane of $N_{r}$. Clearly, there is no polynomial algorithm to differentiate between $B\left(G_{r}\right)$ and $N_{r}$ as any such algorithm will have to go through all the bases/circuits of matroids in both classes. We observe that an oracle also does not simplify the situation at hand and proving that $\mathcal{M}$ contains bicircular matroids, we can surely conclude that there exists no polynomial-time oracle algorithm to test whether a given matroid is bicircular.

We observe that the geometric presentation of $B\left(G_{r}\right)$ is a polygon with $r$ lines where every line has four elements and the loop elements are placed at the vertices. (See Figure 5.1 for an example.)

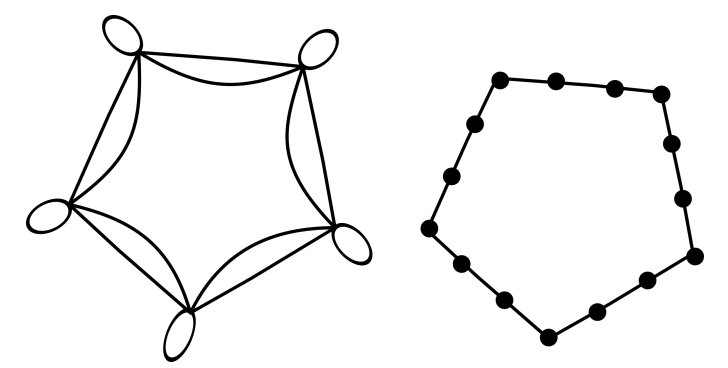

Figure 5.1: The graph $G_{5}$ and geometric representation of $B\left(G_{5}\right)$

In $N_{r}$, one of the bases of $B\left(G_{r}\right)$ is now a circuit and this involves adding an extra circuit in the geometric representation of $B\left(G_{r}\right)$. (See Figure 5.2 for an example.) 


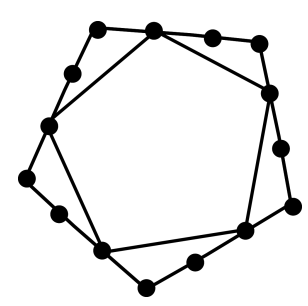

Figure 5.2: $N_{5}$-this is a 4-dimensional picture where the inner polygon forms a circuit-hyperplane of the matroid

Now, in the bicircular representation of $N_{r}$, we have to add a dependent Hamiltonian cycle to $G_{r}$, which is impossible in a graph presentation of a bicircular matroid. Hence, $N_{r}$ is not bicircular. 


\section{Part II}

\section{Well-quasi-ordering}



This part is probably the most intense of all three parts with some beautiful results. Chapter 6 examines why we care about the well-quasi-ordering property and illustrates how it is a focal point of some of the best results in the past decades. We end this chapter by proving that bicircular matroids are well-quasi-ordered basically due to the fact that graphs are well-quasi-ordered.

In Chapter 6, we prove that lattice-path matroids are not well-quasi-ordered. In Chapters 7 and 8 however, we find subclasses of lattice-path matroids that are well-quasi-ordered. In Chapter 7, we introduce square-width - a notion similar to branch-width - and prove that any class of lattice-path matroids with bounded square-width is well-quasi-ordered.

In Chapter 8, we investigate the relevance of a subclass of notch matroids - square notch matroids - and prove that they are essentially the only antichain in the class of lattice-path matroids. Hence, avoiding infinitely many of these in a collection of lattice-path matroids would guarantee a well-quasi-ordering. 


\section{Chapter 6}

\section{Well-quasi-ordering}

Well-quasi-ordering is at the heart of major projects undertaken in discrete mathematics in recent years. A quasi-ordering is a relation, say $\leq$, that is reflexive and transitive. A well-quasi-ordering is a quasi-ordering with the property that, if $a_{0}, a_{1}, \ldots$ is an infinite sequence in the set $A$ that we consider, then there exists $i<j$ such that $a_{i} \leq a_{j}$.

A sequence (possibly infinite) $a_{1}, a_{2}, \ldots$ is called an anti-chain when there does not exist $i$ and $j$ such that $a_{i} \leq a_{j}$. Also, a sequence $a_{1}, a_{2}, \ldots$ is infinitely strictly decreasing if $a_{i}>a_{i+1}$ for $i \geq 1$. Equivalently, a quasi-order is a well-quasi-order if and only if there exists no infinite anti-chain or infinite decreasing sequence (see, for example, [9], Prop 12.1.1).

The expansive Robertson-Seymour graph-minors project was a major accomplishment in discrete mathematics. It was a gigantic feat spanning over 500 pages that proved that graphs are well-quasi-ordered under the minor relation.

Recently, Geelen, Gerards and Whittle announced a proof (Theorem 6, [14]) that the class of $F$-representable matroids is well-quasi-ordered under the minor relation, where $F$ is any finite field. This is connected to their proof of Rota's conjecture. One of the crucial steps that edged them closer to the proof of the former conjecture was their 2002 proof [15] that a class of $F$-representable matroids with bounded branch-width is well-quasi-ordered. We will accomplish the same goal for the class of lattice path matroids. 
Observation 6.1.0.1. Transversal matroids, on the other hand, do not behave so well under those same restrictions.

To observe this, consider the well-known polygon matroid anti-chain (Example 14.1.2, [28]) that starts with the matroids represented in Fig6.1.
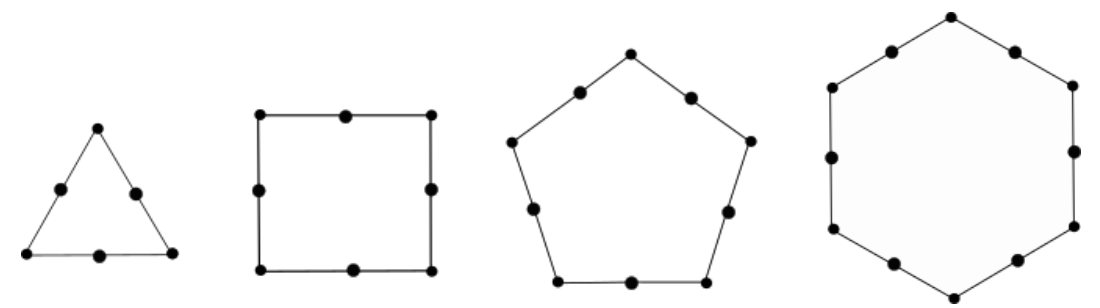

Figure 6.1: The first four matroids in the polygon matroid anti-chain

This is a class of rank-3 matroids, and hence bounded branch-width (at most 3). Since branch-width of the dual of a matroid is same as that of the original matroid, the dual class of this matroid class also has bounded branch-width. Since strict gammoids are exactly the duals of transversal matroids (Corollary 2.4.5, [28]), it is enough to show that this infinite class indeed consists of strict gammoids.

Let $G$ be a directed graph with $X$ and $Y$ being subsets of the vertex set $V$ of $G$. We say that $X$ is linked to $Y$ if $|X|=|Y|$, and there are $|X|$ disjoint directed paths whose initial vertex is in $X$ and final vertex is in $Y$. For $B_{0} \subseteq V$, we say that $X$ is linked into $B_{0}$ if $X$ is linked to a subset of $B_{0}$. When $B_{0}$ is fixed, $L\left(G, B_{0}\right)$ denotes all subsets of $V$ that are linked to $B_{0}$. Then $L\left(G, B_{0}\right)$ is the set of independent sets of a strict gammoid on $V$.

Hence, if we find directed graphs that correspond to the matroid class in Fig 6.1. then we are done. For this, we consider the class of directed graphs as seen in Figure 6.2. In each directed graph, let $B_{0}$ be the set of hollow vertices marked. Then, this is exactly the class of matroids that were represented in Fig 6.1 and we have found an infinite class of transversal matroids of bounded branch-width that form an anti-chain.

As mentioned earlier, lattice path matroids are not well-quasi-ordered. There is a subclass of lattice path matroids named 'notch matroids' by Bonin and de Mier [5]. Their paper includes an excluded minor characterisation for notch matroids (Theorem 8.8, [5]). Among the 

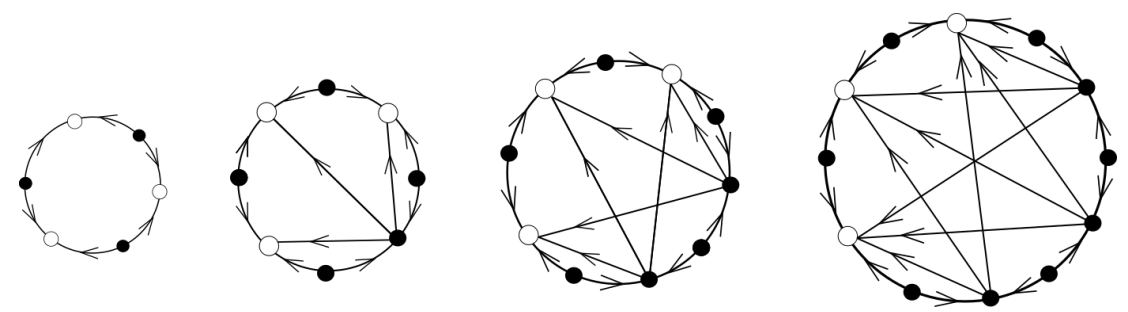

Figure 6.2: Directed graphs corresponding to the polygon matroid anti-chain

excluded minors are three infinite families of lattice path matroids, which are listed below:

- for $n \geq 4$, the rank- $n$ matroid $F_{n}:=T_{n}\left(U_{n-2, n-1} \oplus U_{n-2, n-1}\right)$,

- for $n \geq 2$, the rank- $n$ matroid $G_{n}:=T_{n}\left(U_{n-1, n+1} \oplus U_{n-1, n+1}\right)$, and

- for $n \geq 3$, the rank- $n$ matroid $H_{n}:=T_{n}\left(U_{n-2, n-1} \oplus U_{n-1, n+1}\right)$,

where $T_{n}$ denotes the truncation to rank $n$ (See Fig 6.3). Thus we conclude that these infinite families each form an anti-chain in the class of lattice path matroids and hence the class is not well-quasi-ordered.
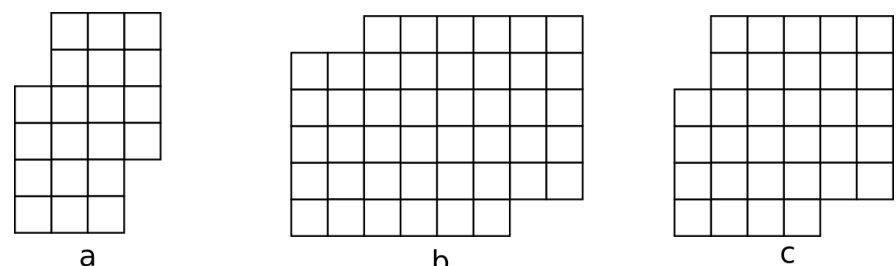

Figure 6.3: (a) $F_{6}\left(\right.$ b) $G_{6}(c) H_{6}$

In Chapters 7 and 8, we prove that imposing certain conditions on the class of lattice path matroids ensures the class is well-quasi-ordered. We would like to note here that the class of bicircular matroids is well-quasi-ordered, primarily due to the fact that they arise from graphs.

Theorem 6.1.1. Bicircular matroids are well-quasi-ordered.

Proof. Let, if possible, there exist an antichain in the class of bicircular matroids, say $B\left(G_{1}\right), B\left(G_{2}\right), \ldots$. Then, consider the corresponding class of graphs $G_{1}, G_{2}, \ldots$. Since this is an infinite collection of finite graphs, 
there exists $G_{i}$ and $G_{j}$, for some $i \neq j$, such that $G_{i}$ is a minor of $G_{j}$. This minor is obtained by the usual graph deletion and contraction. Since, $B(G \backslash e)=B(G) \backslash e$ for any element $e$ and $B(G / e)=B(G) / e$ for any non-loop element $e$, we delete any loop elements in $G_{j}$ that are not in $G_{i}$, following this with deletion and contraction of all non-loop elements in $G_{j}$ that are not in $G_{i}$. Now, $B\left(G_{i}\right)$ is a minor of $B\left(G_{j}\right)$ under the minor relation in bicircular matroids, a contradiction. 


\section{Chapter 7}

\section{Are lattice path matroids well-quasi-ordered?}

\subsubsection{Square-width}

Let $[P, Q]$ be a pair of lattice paths that correspond to the lattice path matroid $M[P, Q]$. We say that $[P, Q]$ is a path presentation that corresponds to the matroid $M[P, Q]$. Recall that the size of a presentation is nothing but the size of the ground set of the corresponding matroid. We use $r$ and $m$, as mentioned previously to denote the rank and co-rank of $M[P, Q]$ respectively. We use $|[P, Q]|$ to denote the number of elements in the presentation $[P, Q]$.

We say that $[P, Q]$ has a $k \times k$ square at $i \in[m+r]$ when $\operatorname{pre}_{i}(P)$ has exactly $k$ more East steps than $\operatorname{pre}_{i}(Q)$. Note that in this case $\operatorname{pre}_{i}(Q)$ has exactly $k$ more North steps than $\operatorname{pre}_{i}(P)$. This corresponds to a $k \times k$ square in the region of the integer lattice bounded by $P$ and $Q$. A $k \times k$ square at $i$ is proper if $i \in[k+1, m+r-k-1]$. We say that $[P, Q]$ has a $k \times k$ square at the top if the last $k$ steps of $P$ are North and the last $k$ steps of $Q$ are East. Similarly, $[P, Q]$ has a $k \times k$ square at the bottom if the first $k$ steps of $P$ are East and those of $Q$ are North.

In Figure 7.1. $P=E E E N E E N N N E N E N$ and $Q=$ NNENNEN ENEEEE, with a $3 \times 3$ square at 7 as $P_{7}=E E E N E E N$ and $Q_{7}=N N E N N E N$. 


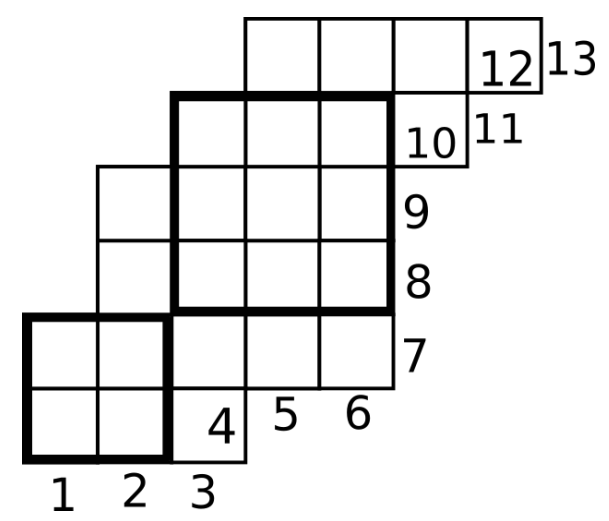

Figure 7.1: The square at 2 is a $2 \times 2$ square whereas the square at 7 is a proper $3 \times 3$ square

A lattice path presentation is said to have square-width $k$ when the largest square it contains is a $k \times k$ square. Square-width is closely associated with branch-width of a matroid as we aim to prove using the next three results. This concept has a key role in conjectures like Rota's Conjecture. We cite the definition of branchwidth as appears in [28].

A tree is subcubic if every vertex that is not a leaf has degree three. A branch-decomposition of a matroid $M$ consists of a cubic tree $T$ with at least $|E(M)|$ leaves together with a labelling of $|E(M)|$ leaves by different elements of $E(M)$. For each edge $e$ of such a branch-decomposition $T$ of $M$, the graph $T \backslash e$ has exactly two components. Thus the set of labelled leaves of $T$, and hence $E(M)$, is partitioned into two subsets $X$ and $E(M)-X$, say. We say $X$ and $E(M)-X$ are displayed by the edge $e$. The width $w(e)$ of the edge $e$ is defined to be $w(e)=r(X)+r(E-X)-r(M)+1$. The width $w(T)$ of the tree $T$ is the maximum of the widths of the edges of $T$. The branch-width of $M, b w(M)$ is the minimum of the width of $T$ over all such labelled cubic trees $T$.

Lemma 7.1.1. Let $[P, Q]$ be a path presentation with a $k \times k$ square. Then $M[P, Q]$ has a $U_{k, 2 k}$-minor.

Proof. Let $M[P, Q]$ be a minimal counter-example to our hypothesis, with $P$ and $Q$ being lattice paths from $(0,0)$ to $(m, r)$. Let the corners of the $k \times k$ square be at $(i, j),(i+k, j),(i, j+k)$ and $(i+k, j+k)$. If $i>0$, then the first element is not part of the $k \times k$ square. This implies that $M[P, Q] \backslash 1$ contains a $k \times k$ square, which in turn implies that $M[P, Q] \backslash 1$ 
contains a $U_{k, 2 k}$-minor. But then so would $M[P, Q]$, which contradicts our assumption. Thus $i=0$. Similarly, $j=0$ as otherwise, $M[P, Q] / 1$ would contain a $k \times k$ square.

Now, if $2 k<m+r$, the path presentation of either $M[P, Q] \backslash m+r$ or $M[P, Q] / m+r$ contains a $k \times k$ square, which again leads to a contradiction. Hence, $2 k=m+r$. Also, since $k \leq \min \{m, r\}$, it follows that $m=r=k$. Thus $P$ and $Q$ bound a $k \times k$ square, and so $M[P, Q]$ is in fact isomorphic to $U_{k, 2 k}$ and the proof is complete.

Corollary 7.1.2. Let $M=M[P, Q]$ be a lattice path matroid and assume that $b w(M) \leq k$. Then the square-width of $[P, Q]$ is less than $\lceil 3 k / 2\rceil$.

Proof. Assume that the square width of $[P, Q]$, say $j$, is at least $\lceil 3 k / 2\rceil$. Then by Lemma 7.1.1, $M[P, Q]$ has a $U_{j, 2 j}$ minor. But the branch-width of $U_{j, 2 j}$ is $\lceil 2 j / 3\rceil+1$ (Exercise 14.2.5, [28]), and hence the branch-width of $U_{j, 2 j}$ is at least $k+1$. But this is a contradiction to our assumption that the branch-width of $M[P, Q]$ is at most $k$.

Lemma 7.1.3. Let $M[P, Q]$ be a lattice path matroid, where the largest proper square of $[P, Q]$ has size $k \times k$. Then bw $(M) \leq k+1$.

Proof. Consider the branch decomposition of $M([P, Q])$ represented in Fig 7.2 .

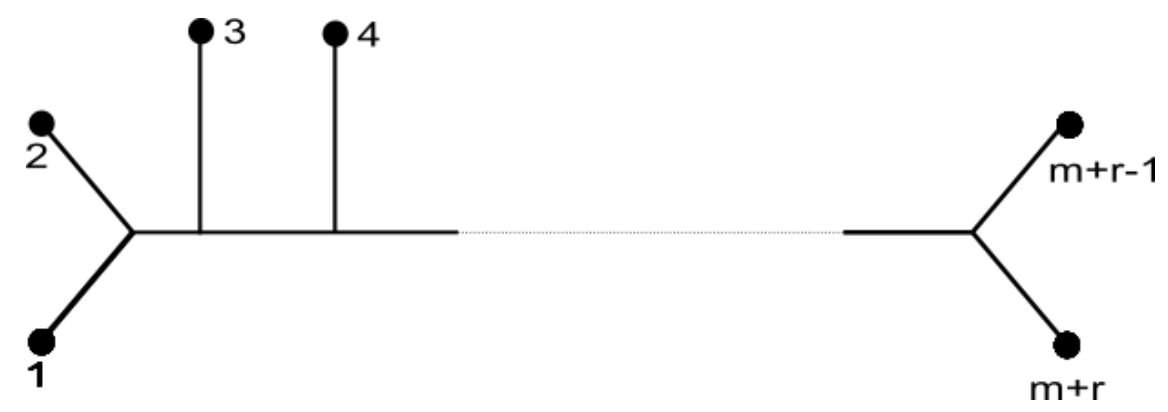

Figure 7.2: A branch decomposition of $M[P, Q]$

The displayed sets are all of the form $\{1, \ldots, i\}$ or $\{i+1, \ldots, m+r\}$. Then

$$
\begin{gathered}
r([1, i]) \leq \text { number of North steps in } \operatorname{pre}_{i}(Q), \text { and } \\
r([i+1, m+r]) \leq \text { number of North steps in } \operatorname{suf}_{i}(P) .
\end{gathered}
$$


Also $r(M[P, Q])=$ number of North steps in $P$. Then the connectivity function $\lambda$ is given by

$$
\begin{aligned}
\lambda([1, i]) & =r([1, i])+r([i+1, m+r])-r(M[P, Q]) \\
& \leq \text { number of North steps in } \operatorname{pre}_{i}(Q)+\text { number of North steps in } \operatorname{suf}_{i}(P) \\
& \quad-\text { number of North steps in } \mathrm{P} \\
& \leq k, \quad
\end{aligned}
$$

or else there is a square larger than $k \times k$. Since $b w(M[P, Q])=\min$ (width) over all branch decompositions of $M[P, Q]$, we conclude that $b w(M[P, Q]) \leq k+1$.

Combining the above results, we obtain the following corollary:

Corollary 7.1.4. A class of lattice path matroids has bounded branch-width if and only if the corresponding class of presentations has bounded square-width.

Definition 7.1.5. Let $[P, Q]$ be a lattice path presentation on $[m+r]$ with a proper $k \times k$ square at $i$. Then we define two new lattice path presentations from $[P, Q]$ as follows:

$$
\begin{aligned}
B_{i}(P) & =\operatorname{pre}_{i}(P) N^{k} \\
B_{i}(Q) & =\operatorname{pre}_{i}(Q) E^{k}
\end{aligned}
$$

and

$$
\begin{aligned}
T_{i}(P) & =E^{k} \operatorname{suf}_{i}(P) \text { and } \\
T_{i}(Q) & =N^{k} \operatorname{suf}_{i}(Q) .
\end{aligned}
$$

Note that $M\left(\left[B_{i}(P), B_{i}(Q)\right]\right)$ is a lattice path matroid on the ground set $[i+k]$ but we relabel $M\left(\left[T_{i}(P), T_{i}(Q)\right]\right)$ to be a lattice path matroid on the ground set $[i-k, m+r]$, so as to retain the same elements as in the original matroid. This will prove beneficial in the gluing operation that follows. In Figure 7.3. $\operatorname{pre}_{7}(P)=E E E N E E N, \operatorname{pre}_{7}(Q)=N N E N N E N, \operatorname{suf}_{7}(P)=$ NNENEN and $\operatorname{suf}_{7}(Q)=$ ENEEEE. Thus $B_{7}(P)=\operatorname{pre}_{7}(P) N^{3}, B_{7}(Q)=$ $\operatorname{pre}_{7}(Q) E^{3}, T_{7}(P)=E^{3} \operatorname{suf}_{7}(P)$ and $T_{7}(Q)=N^{3} \operatorname{suf}_{7}(Q)$. 


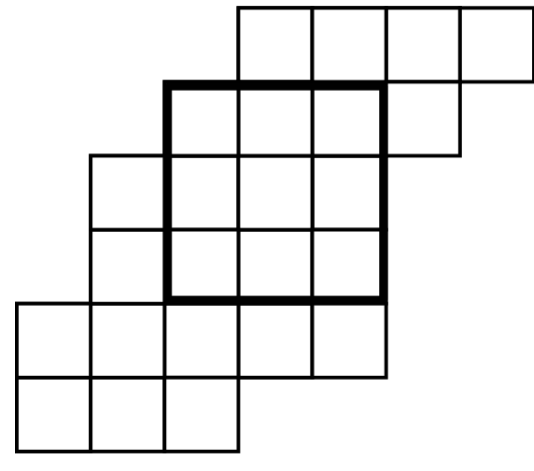

(a)
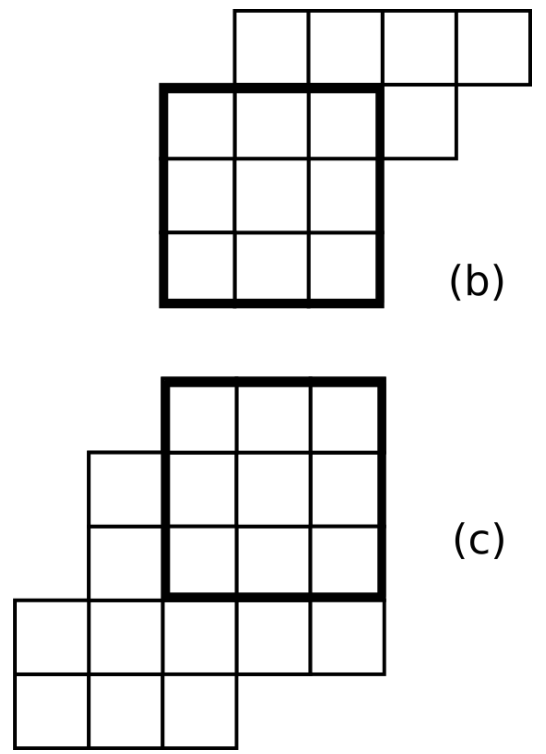

(c)

Figure 7.3: (a) A proper $k \times k$ square in $[P, Q]$ at 7 (b) $\left[T_{7}(P), T_{7}(Q)\right]$ (c) $\left[B_{i}(P), B_{i}(Q)\right]$

An intuitive property of these new path presentations is proved below :

Lemma 7.1.6. Let $\left[B_{i}(P), B_{i}(Q)\right]$ and $\left[T_{i}(P), T_{i}(Q)\right]$ be as in Definition 7.1.5 Then $\left[B_{i}(P), B_{i}(Q)\right],\left[T_{i}(P), T_{i}(Q)\right]$ are minors of $[P, Q]$.

Proof. We know that $P=\operatorname{pre}_{i}(P) \operatorname{suf}_{i}(P)$ and $Q=\operatorname{pre}_{i}(Q) \operatorname{suf}_{i}(Q)$, where $1<i<m+r$. Since there is a $k \times k$ square at $i, \operatorname{pre}_{i}(P)$ has $k$ more East steps than $\operatorname{pre}_{i}(Q)$. Hence, $\operatorname{suf}_{i}(Q)$ has $k$ more East steps than $\operatorname{suf}_{i}(P)$. Similarly, $\operatorname{suf}_{i}(P)$ has $k$ more North steps than $\operatorname{suf}_{i}(Q)$. Recall that deletion requires the removal of East steps from both paths, and contraction requires removal of North steps from both paths.

Let $J$ be the subset of $\{i+1, \ldots, m+r\}$ such that $P$ contains $E$ at exactly those steps. Let $\left[P^{\prime}, Q^{\prime}\right]$ be $[P, Q] \backslash J$. Then, $\operatorname{suf}_{i}\left(P^{\prime}\right)$ contains no East steps but $\operatorname{suf}_{i}\left(Q^{\prime}\right)$ has exactly $k$. Now let $I$ be the set of positions after $i$ where $Q^{\prime}$ contains $N$. Define $\left[P^{\prime \prime}, Q^{\prime \prime}\right]$ to be $\left[P^{\prime}, Q^{\prime}\right] / I$. So $\operatorname{suf}_{i}\left(Q^{\prime \prime}\right)$ contains no North steps and $\operatorname{suf}_{i}\left(P^{\prime \prime}\right)$ contains exactly $k$. Hence, $\left[P^{\prime \prime}, Q^{\prime \prime}\right]=$ $\left[\operatorname{pre}_{i}(P) N^{k}, \operatorname{pre}_{i}(Q) E^{k}\right]$. Then $\left[P^{\prime \prime}, Q^{\prime \prime}\right]$ is nothing but $\left[B_{i}(P), B_{i}(Q)\right]$.

The same deductions as above imply that $\left[T_{i}(P), T_{i}(Q)\right]$ is a minor of $[P, Q]$.

Definition 7.1.7. If we have a pair of lattice path presentations $\left[P_{B}, Q_{B}\right]$ and $\left[P_{T}, Q_{T}\right]$ such that the following conditions are satisfied: 
(i) The last $k$ steps in $P_{B}$ are North steps, that is, $P_{B}=P_{B}^{\prime} N^{k}$

(ii) The last $k$ steps in $Q_{B}$ are East steps, that is, $Q_{B}=Q_{B}^{\prime} E^{k}$

(iii) The first $k$ steps in $P_{T}$ are East steps, that is, $P_{T}=E^{k} P_{T}^{\prime}$

(iv) The first $k$ steps in $Q_{T}$ are North steps, that is, $Q_{T}=N^{k} Q_{T}^{\prime}$.

Then we define $G L\left(\left[P_{B}, Q_{B}\right],\left[P_{T}, Q_{T}\right]\right)$ to be $[P, Q]$, where $P=P_{B}^{\prime} P_{T}^{\prime}$, and $Q=Q_{B}^{\prime} Q_{T}^{\prime}$. When they are defined as above, we say that $\left[P_{B}, Q_{B}\right]$ has a $k \times k$ square at the top and $\left[P_{T}, Q_{T}\right]$ has a $k \times k$ square at the bottom and that $G L\left(\left[P_{B}, Q_{B}\right],\left[P_{T}, Q_{T}\right]\right)$ is obtained by gluing $\left[P_{B}, Q_{B}\right]$ and $\left[P_{T}, Q_{T}\right]$.

In other words, if we start with a lattice path $[P, Q]$ and 'pull them apart' at a $k \times k$ square to give rise to two new lattice paths $\left[B_{i}(P), B_{i}(Q)\right]$ and $\left[T_{i}(P), T_{i}(Q)\right]$, then $G L\left(\left[B_{i}(P), B_{i}(Q)\right],\left[T_{i}(P), T_{i}(Q)\right]\right)$ will lead us back to the lattice path $[P, Q]$ that we originally had. This fact is illustrated in the following lemma:

Lemma 7.1.8. Let $[P, Q]$ be a path presentation with a $k \times k$ square at $i$, where $P=\operatorname{pre}_{i}(P) \operatorname{suf}_{i}(P)$ and $Q=\operatorname{pre}_{i}(Q) \operatorname{suf}_{i}(Q)$. Construct the two lattice paths $\left[B_{i}(P), B_{i}(Q)\right]$ and $\left[T_{i}(P), T_{i}(Q)\right]$. Then $G L\left(\left[B_{i}(P), B_{i}(Q)\right],\left[T_{i}(P), T_{i}(Q)\right]\right)=$ $[P, Q]$.

Proof. By Definition 7.1.5, paths $B_{i}(P), B_{i}(Q), T_{i}(P)$ and $T_{i}(Q)$ satisfy conditions (i) - (iv) in Definition 7.1.7. Thus $P_{B}^{\prime}=\operatorname{pre}_{i}(P), Q_{B}^{\prime}=\operatorname{suf}_{i}(P)$, $P_{T}^{\prime}=\operatorname{suf}_{i}(P)$ and $Q_{T}^{\prime}=\operatorname{suf}_{i}(Q)$. Thus $P_{B}^{\prime} P_{T}^{\prime}=\operatorname{pre}_{i}(P) \operatorname{suf}_{i}(P)=P$ and $Q_{B}^{\prime} Q_{T}^{\prime}=\operatorname{pre}_{i}(Q) \operatorname{suf}_{i}(Q)=Q$. This completes the proof.

\subsubsection{The importance of square-width}

A square presentation is a lattice path presentation where $P=E^{r} N^{r}$ and $Q=N^{r} E^{r}$. Observe that a square presentation does not have a proper square.

We say an element in a matroid is protected when we do not allow that element to be deleted or contracted to produce a minor. 
Definition 7.1.9. Let $k$ be a positive integer. Let $\mathcal{U}_{k}$ be the class of all triples $(L, t, b)$, where $L=[P, Q]$ is a lattice-path presentation of square-width at most $k$ on $\{1, \ldots, m+r\}$ and $\{t, b\} \in\{0,1\}$.

The definition can be interpreted as $t=1$ when the top (or last) $k$ elements are protected and $b=1$ when the bottom (or first) $k$ elements are protected.

We say that $(L, t, b)$ is a lattice triple. The dual of $(L, t, b)$ is $\left(L^{*}, t, b\right)$ where $L^{*}$ is the dual of $L$. Then $(L, t, b)$ is in $\mathcal{U}_{k}$ if and only if $\left(L^{*}, t, b\right)$ is in $\mathcal{U}_{k}$.

Definition 7.1.10. Let $(L, t, b)$ and $\left(L^{\prime}, t^{\prime}, b^{\prime}\right)$ be lattice triples in $\mathcal{U}_{k}$, where $L$ has rank $r$ and co-rank $m$. We say that $\left(L^{\prime}, t^{\prime}, b^{\prime}\right) \preceq(L, t, b)$, that is, $\left(L^{\prime}, t^{\prime}, b^{\prime}\right)$ is a triple minor of $(L, t, b)$, if there exists disjoint subsets $I, J$ of $\{1, \ldots, m+r\}$ such that $L^{\prime}$ is isomorphic to $L / I \backslash J$ and if $t=1, I \cup J$ contains none of the last $k$ elements and if $b=1, I \cup J$ contains none of the first $k$ elements.

We now define the gluing together operation for lattice triples as follows:

Definition 7.1.11. Let $\left[P_{B}, Q_{B}\right]$ and $\left[P_{T}, Q_{T}\right]$ be defined such that $G L\left(\left[P_{B}, Q_{B}\right],\left[P_{T}, Q_{T}\right]\right)$ is well-defined. If $B=\left(\left[P_{B}, Q_{B}\right], t_{B}, b_{B}\right)$ and $T=\left(\left[P_{T}, Q_{T}\right], t_{T}, b_{T}\right)$ are lattice triples, then $G L(B, T)$ is defined to be the lattice triple $\left(G L\left(\left[P_{B}, Q_{B}\right],\left[P_{T}, Q_{T}\right]\right), t_{T}, b_{B}\right)$.

We are now well-equipped to prove a central lemma.

Lemma 7.1.12. Let $[P, Q]$ be a path presentation with a proper $k \times k$ square at $i$ and let $L$ be the lattice triple $\left([P, Q], t_{L}, b_{L}\right)$ in $\mathcal{U}_{k}$. Let $\left[B_{i}(P), B_{i}(Q)\right]$ and $\left[T_{i}(P), T_{i}(Q)\right]$ be as in Definition 7.1.5 and $B, T$ be the lattice triples $\left(\left[B_{i}(P), B_{i}(Q)\right], t_{B}, b_{B}\right)$ and $\left(\left[T_{i}(P), T_{i}(Q)\right], t_{T}, b_{T}\right)$ respectively, where $t_{B}=$ $b_{T}=1, b_{B}=b_{L}$ and $t_{T}=t_{L}$. Let $B^{\prime}=\left(\left[P_{B}, Q_{B}\right], t_{B^{\prime}}, b_{B^{\prime}}\right)$ and $T^{\prime}=$ $\left(\left[P_{T}, Q_{T}\right], t_{T^{\prime}}, b_{T^{\prime}}\right)$ be triple minors of $B$ and $T$ respectively and be in $\mathcal{U}_{k}$ with a $k \times k$ square at the bottom and top respectively. Then $G L\left(\left[B^{\prime}, T^{\prime}\right]\right)$ is a triple minor of $L$.

Proof. We prove this by induction on $n$, where $n$ is the sum of size differences of $\left\{B, B^{\prime}\right\}$ and $\left\{T, T^{\prime}\right\}$. Note that $\left[B_{i}(P), B_{i}(Q)\right]$ has a $k \times k$ square at the top and $\left[T_{i}(P), T_{i}(Q)\right]$, has a $k \times k$ square at the bottom as $t_{B}=b_{T}=1$ and they are both in $\mathcal{U}_{k}$. 
When $n=1$, either $\left\{\left[P_{B}, Q_{B}\right],\left[B_{i}(P), B_{i}(Q)\right]\right\}$ or $\left\{\left[P_{T}, Q_{T}\right],\left[T_{i}(P), T_{i}(Q)\right]\right\}$ differ by a single element in the ground set. Let the former be true, in which case, an element, say $e$, was deleted or contracted from $\left[B_{i}(P), B_{i}(Q)\right]$ to obtain $\left[P_{B}, Q_{B}\right]$. If $\left[P_{T}, Q_{T}\right]$ and $\left[T_{i}(P), T_{i}(Q)\right]$ differ by a single element, the same reasoning can be used to see that for $e>i$, the same operation can be carried out after gluing. By duality, the case when $e$ is contracted is similar to when $e$ is deleted. So we can assume that $e$ was deleted. Now, since $t_{B}=1, e<i$ and $e$ is a step in the sub-strings $\operatorname{pre}_{i}(P)$ and $\operatorname{pre}_{i}(Q)$. Also, when $b_{B}=1, k<e \leq i$. We consider the different cases where $e$ is a North step and an East step in the sub-strings $\operatorname{pre}_{i}(P)$ and $\operatorname{pre}_{i}(Q)$.

Case (i): $e$ is an East step in both sub-strings

We can decompose the paths as $P=P_{0} E P_{1} \operatorname{suf}_{i}(P)$ and $Q=$ $Q_{0} E Q_{1} \operatorname{suf}_{i}(Q)$, where $P_{0} E P_{1}=\operatorname{pre}_{i}(P), Q_{0} E Q_{1}=\operatorname{pre}_{i}(Q)$ and the East steps represent the $e^{\text {th }}$ position. Then, it is obvious that we have to remove the East steps from both paths. Thus,

$$
\left[B_{i}(P), B_{i}(Q)\right]=\left[\operatorname{pre}_{i}(P) N^{k}, \operatorname{pre}_{i}(Q) E^{k}\right]=\left[P_{0} E P_{1} N^{k}, Q_{0} E Q_{1} E^{k}\right] .
$$

Therefore,

$$
\left[B_{i}(P), B_{i}(Q)\right] \backslash e=\left[P_{0} P_{1} N^{k}, Q_{0} Q_{1} E^{k}\right]=\left[P_{B}, Q_{B}\right] .
$$

Also,

$$
\left[P_{T}, Q_{T}\right]=\left[T_{i}(P), T_{i}(Q)\right]=\left[E^{k} \operatorname{suf}_{i}(P), N^{k} \operatorname{suf}_{i}(Q)\right]
$$

Hence,

$$
G L\left(\left[P_{B}, Q_{B}\right],\left[P_{T}, Q_{T}\right]\right)=\left[P_{0} P_{1} \operatorname{suf}_{i}(P), Q_{0} Q_{1} \operatorname{suf}_{i}(Q)\right] .
$$

On the other hand,

$$
\begin{gathered}
{[P, Q]=\left[P_{0} E_{1} \operatorname{suf}_{i}(P), Q_{0} E Q_{1} \operatorname{suf}_{i}(Q)\right] \text { and thus }} \\
{[P, Q] \backslash e=\left[P_{0} P_{1} \operatorname{suf}_{i}(P), Q_{0} Q_{1} \operatorname{suf}_{i}(Q)\right]=G L\left(\left[P_{B}, Q_{B}\right],\left[P_{T}, Q_{T}\right]\right) .}
\end{gathered}
$$




$$
\therefore G L\left(B^{\prime}, T^{\prime}\right)=\left(G L\left(\left[P_{B}, Q_{B}\right],\left[P_{T}, Q_{T}\right]\right), t_{T^{\prime}}, b_{B^{\prime}}\right) \preceq\left([P, Q], t_{L}, b_{L}\right)
$$

Case (ii): $e$ is an East step in $P$, but a North step in $Q$ The $\left[B_{i}(P), B_{i}(Q)\right]$ in this case can be presented as :

$$
\left[B_{i}(P), B_{i}(Q)\right]=\left[\operatorname{pre}_{i}(P) N^{k}, \operatorname{pre}_{i}(Q) E^{k}\right]=\left[P_{0} E P_{1} N^{k}, Q_{0} N Q_{1} E^{k}\right] .
$$

Clearly, it is enough to remove the East step from $P$. Now, $Q=$ $Q_{0} N Q_{1} \operatorname{suf}_{i}(Q)$. Then, $Q_{1}$ will contain an East step. Else, suppose that $Q_{1}$ does not contain any East step. Then, $\operatorname{pre}_{i}(Q)=Q_{0} N \ldots N$ and $B_{i}(Q)=Q_{0} N \ldots N E^{k}$. Now, when we delete $e$ from $\operatorname{pre}_{i}(Q)$, we are essentially removing an East step from the last $k$ East steps in $B_{i}(Q)$. But this is impossible as $Q_{B}$ has a $k \times k$ square at the top and thus the last $k$ steps are East steps. Thus we will be able to find an East step in $Q_{1}$ which can be removed. Then we rewrite $\operatorname{pre}_{i}(Q)$ as $\operatorname{pre}_{i}(Q)=Q_{0}^{\prime} E Q_{1}^{\prime}$, where $E$ is the first East step in $Q_{1}$. Hence,

$$
\begin{gathered}
{\left[B_{i}(P), B_{i}(Q)\right]=\left[P_{0} E P_{1} N^{k}, Q_{0}^{\prime} E Q_{1}^{\prime} E^{k}\right] \text { and }} \\
{\left[B_{i}(P), B_{i}(Q)\right] \backslash e=\left[P_{0} P_{1} N^{k}, Q_{0}^{\prime} Q_{1}^{\prime} E^{k}\right] .}
\end{gathered}
$$

Similar to the case above,

$$
G L\left(\left[P_{B}, Q_{B}\right],\left[P_{T}, Q_{T}\right]\right)=\left[P_{0} P_{1} \operatorname{suf}_{i}(P)^{\prime}, Q_{0}^{\prime} Q_{1}^{\prime} \operatorname{suf}_{i}(Q)\right] .
$$

Also,

$$
[P, Q] \backslash e=\left[P_{0} P_{1} \operatorname{suf}_{i}(P)^{\prime}, Q_{0}^{\prime} Q_{1}^{\prime} \operatorname{suf}_{i}(Q)\right]=G L\left(\left[P_{B}, Q_{B}\right],\left[P_{T}, Q_{T}\right]\right) .
$$

Case (iii): $e$ is a North step in $P$, but an East step in $Q$

Similar to the argument above, if $\operatorname{pre}_{i}(P)=P_{0} N P_{1}$, then $P_{0}$ will have an East step. If $P_{0}$ has no east step, then $\operatorname{pre}_{i}(P)$ is $N \ldots N P_{1}$, which means that the first $i$ steps are coloops and thus $Q$ has no East step at $i$ or before it. This contradicts our hypothesis. Note that, if $b_{B}=1$, then this East step is a position greater than $i$ in $Q$. Thus, rewriting $\operatorname{pre}_{i}(P)$ as $P_{0}^{\prime} E P_{1}^{\prime}$, where 
$E$ is the last East step in $P_{0}$, we obtain that

$$
[P, Q] \backslash e=\left[P_{0}^{\prime} P_{1}^{\prime} \operatorname{suf}_{i}(P), Q_{0} Q_{1} \operatorname{suf}_{i}(Q)\right]=G L\left(\left[P_{B}, Q_{B}\right],\left[P_{T}, Q_{T}\right]\right) .
$$

Case (iv): $e$ is a North step in both $P$ and $Q$

Suppose, $P_{0}$ does not each contain an East step, where $\operatorname{pre}_{i}(P)=P_{0} N P_{1}$ and $\operatorname{pre}_{i}(Q)=Q_{0} N Q_{1}$. Then $Q_{0}$ also does not contain an East step. But this implies that the North step at position $e$ is a co-loop. Since deletion of a co-loop involves deletion of the corresponding North step from both paths, we can remove $e$ from both $\operatorname{pre}_{i}(P)$ and $\operatorname{pre}_{i}(Q)$. Then,

$$
\begin{gathered}
{\left[B_{i}(P), B_{i}(Q)\right] \backslash e=\left[P_{0} P_{1} N^{k}, Q_{0} Q_{1} E^{k}\right], \text { and }} \\
G L\left(\left[P_{B}, Q_{B}\right],\left[P_{T}, Q_{T}\right]\right)=\left[P_{0} P_{1} \operatorname{suf}_{i}(P), Q_{0} Q_{1} \operatorname{suf}_{i}(Q)\right]=[P, Q] \backslash e .
\end{gathered}
$$

If $P_{0}$ does contain an East step, then so should $Q_{1}$, as argued in the preceding cases, and deletion would be removal of the last East step in $P_{0}$ and first East step in $Q_{1}$. Then rewriting $\operatorname{pre}_{i}(P)$ as $P_{0}^{\prime} E P_{1}^{\prime}$ and $\operatorname{pre}_{i}(Q)$ as $Q_{0}^{\prime} E Q_{1}^{\prime}$ (following the convention above), we obtain

$$
\begin{gathered}
{\left[B_{i}(P), B_{i}(Q)\right] \backslash e=\left[P_{0}^{\prime} P_{1}^{\prime} N^{k}, Q_{0}^{\prime} Q_{1}^{\prime} E^{k}\right]=\left[P_{B}, Q_{B}\right] \text { and }} \\
G L\left(\left[P_{B}, Q_{B}\right],\left[P_{T}, Q_{T}\right]\right)=\left[P_{0}^{\prime} P_{1}^{\prime} \operatorname{suf}_{i}(P), Q_{0}^{\prime} Q_{1}^{\prime} \operatorname{suf}_{i}(Q)\right]=[P, Q] \backslash e .
\end{gathered}
$$

Thus we conclude that when $e<i, G L\left(\left[P_{B}, Q_{B}\right],\left[P_{T}, Q_{T}\right]\right)$ is a minor of $[P, Q]$.

So the result holds true when $n=1$. Assume that it holds true for $n=j$. We prove the result for $n=j+1$.

Let $B, T, B^{\prime}$ and $T^{\prime}$ be as described in the statement of the lemma. We then need to prove that $G L\left(B^{\prime}, T^{\prime}\right)$ is a minor of $G L(B, T)$. Since $B^{\prime}, T^{\prime}$ are minors of $B, T$ respectively, $\left[P_{B}, Q_{B}\right]=\left[B_{i}(P), B_{i}(Q)\right] \backslash I / J$, for disjoint sets $I$ and $J$. Also, $\left[P_{T}, Q_{T}\right]=\left[T_{i}(P), T_{i}(Q)\right] \backslash I^{\prime} / J^{\prime}$ for disjoint sets $I^{\prime}$ and $J^{\prime}$. 
Since

$$
\left(|B|-\left|B^{\prime}\right|\right)+\left(|T|-\left|T^{\prime}\right|\right)=j+1,
$$

one of $I, J, I^{\prime}, J^{\prime}$ is non-empty. Without loss of generality, let $I$ be non-empty as the cases when $J, I^{\prime}$ or $J^{\prime}$ are non-empty are identical.

Let $e \in I$. Then

$$
(|B|-|B \backslash e|)+(|T|-|T|)=1
$$

and hence by the case proved for when $n=1, G L(B \backslash e, T)$ is a minor of $G L(B, T)$. Also,

$$
\left(|B \backslash e|-\left|B^{\prime}\right|\right)+\left(|T|-\left|T^{\prime}\right|\right)<j+1 .
$$

Thus, by our induction hypothesis, $G L\left(B^{\prime}, T^{\prime}\right)$ is a minor of $G L(B \backslash e, T)$ which is already a minor of $G L(B, T)$. This finishes the proof for the case $n=j+1$ and completes our induction argument.

With the aid of the above lemma, we are now ready to prove that the class of lattice path matroids with bounded square-width is well-quasi-ordered. We use a minimal bad sequence argument in the proof of the same. An infinite sequence $a_{1}, a_{2}, a_{3}, \ldots$ is bad if there does not exist an $i$ and $j$ such that $a_{i} \leq a_{j}$. Otherwise, the sequence is good. Also, a subsequence $\left(a_{i_{1}}, a_{i_{2}}, \ldots\right)$ is bad if it is a bad sequence. In essence, a graph class or matroid class is well-quasi-ordered if and only if it does not have a bad sequence, as graph and matroid classes do not contain infinite decreasing sequences. We require a lemma about bad sequences to explain the minimal bad sequence argument.

Lemma 7.1.13. Let $a_{1}, a_{2}, \ldots$, be an infinite sequence with no bad subsequences. Then there exists $i_{1}<i_{2}<i_{3}<\ldots$, such that $a_{i_{s}} \leq a_{i_{s+1}}$ for every s.

Proof. We begin by constructing a directed graph as follows: if $a_{i_{s}}<a_{i_{t}}$ and there does not exist a $k$ such that $s, t \neq k$ and $a_{i_{s}} \leq a_{i_{k}} \leq a_{i_{t}}$, then we have a directed edge from vertex $a_{i_{s}}$ to vertex $a_{i_{t}}$.

Now, $G$ has to be a directed graph with finitely many connected components. Else, suppose that $G$ has infinitely many connected components $G_{1}, G_{2}, \ldots$. Then selecting a vertex from each component 
provides us with infinitely many vertices $a_{i_{1}}, a_{i_{2}}, \ldots$ such that there does not exist $s, t$ where $a_{i_{s}} \leq a_{i_{t}}$, which contradicts our assumption that $a_{1}, a_{2}, \ldots$, does not have a bad subsequence.

Clearly, at least one among the finite components of $G$ must have infinite number of vertices. Hence, by König's Lemma [21](see also [11]), this infinite graph either contains a vertex of infinite degree or an infinite simple path. If there exists such a vertex, then the elements corresponding to the adjacent infinite number of vertices form a bad sequence. Thus $G$ cannot contain a vertex of infinite degree. Hence it contains a simple path which completes our proof.

This minimal bad sequence argument led Nash-Williams [27] to a very elegant proof for the following theorem:

Theorem 7.1.14. Finite trees are well-quasi-ordered with respect to the graph minor relation.

The proof is briefly sketched here:

Proof. Suppose the class of finite trees is not well-quasi-ordered. Then there exists a bad sequence of trees. Let $T_{1}$ be the smallest tree that can start such a sequence. Following this we choose $T_{2}$ to be the smallest tree in position 2 among all sequences that start with $T_{1}$, and hence $T_{k}$ will be the smallest tree in position $k$ among all sequences that start with $T_{1}, T_{2}, \ldots$ Then $T_{1}, T_{2}, \ldots$ is a bad sequence too.

Now, let $T_{i}^{\prime}$ be the tree obtained from $T_{i}$ by deleting any branch from the root, and $T_{i}^{\prime \prime}$ be the branch deleted. Since $T_{1}, T_{2}, \ldots$ is the minimal bad sequence $T_{1}^{\prime}, T_{2}^{\prime}, \ldots$ does not contain a bad subsequence. Hence by Lemma 7.1.13 there exists $T_{i_{1}}, T_{i_{2}}, \ldots$ such that $T_{i_{s}} \leq T_{i_{s}+1}$. Similarly $T_{1}^{\prime \prime}, T_{2}^{\prime \prime}, \ldots$ is not contain a bad subsequence. Thus there exists $s<t$ such that $T_{i_{s}}^{\prime \prime} \leq T_{i_{t}}^{\prime \prime}$. But this implies that $T_{i_{s}}^{\prime} \leq T_{i_{t}}^{\prime}$ and hence $T_{i_{s}} \leq T_{i_{t}}$, which is a contradiction.

For an arbitrary set $\Sigma$, let $\Sigma^{*}$ be the set of all finite sequences of $\boldsymbol{\Sigma}$. Any quasi-order $\leq$ on $\Sigma$ defines a quasi-order $\preceq$ on $\Sigma^{*}$ as follows: $\left(a_{1}, a_{2}, \ldots, a_{m}\right) \preceq\left(b_{1}, b_{2}, \ldots, b_{n}\right)$ if and only if there is an order-preserving 
injection $f:\left\{a_{1}, \ldots, a_{m}\right\} \rightarrow\left\{b_{1}, \ldots, b_{n}\right\}$ with $a_{i} \leq f\left(a_{i}\right)$ for each $i$. Then Higman's Lemma [18] states that $\left(\boldsymbol{\Sigma}^{*}, \preceq\right)$ is a well-quasi-order if $(\boldsymbol{\Sigma}, \leq)$ is a well-quasi-order.

A nested matroid is a matroid that can be obtained from the empty matroid by iterating the operations of adding isthmuses and taking free extensions. Thus nested matroids are also lattice path matroids $M[P, Q]$ with presentations that can be represented as $[P, Q]=\left[E^{m} N^{r}, Q\right]$ when $M$ is a rank-r matroid with co-rank $m$. We use $M([Q])$ to denote a nested matroid $M\left(\left[E^{m} N^{r}, Q\right]\right)$ and omit the lower bounding path. Nested matroids form a well-quasi-ordered class [4] and we further prove the following lemma:

Lemma 7.1.15. Let $C$ be the set of triples $([Q], t, b)$ where $M[Q]$ is a nested matroid. Then $C$ is well-quasi-ordered under $\preceq$.

Proof. If $M[Q]$ is nested, then we can assume that $P=E^{m} N^{r}$ for non-negative integers $m$ and $r$. Let $Q$ and $Q^{\prime}$ be lower bounding paths which implies that they are strings made up of $N$ and $E$ steps. If $Q^{\prime}$ is a subsequence of $Q$, then $\left[Q^{\prime}\right]$ is a minor of $[Q]$.

Consider an infinite antichain in $C$. Since there are only $2^{k}$ possible starting and ending $k$-character strings, we can assume that infinitely many sequences in the antichain start and end with the same $k$ characters. But binary strings are well-quasi-ordered under the subsequence relation, by an application of Higman's Lemma [18]. Therefore, there are two sequences in the antichain, $Q^{\prime}$ and $Q$, where $Q^{\prime}$ is a subsequence of $Q$, and they have the same first and last $k$ characters. By contracting any $N$ in $Q$ but not in $Q^{\prime}$, and deleting any $E$ in $Q$ but not $Q^{\prime}$, we see that $\left[Q^{\prime}\right]$ is a minor of $[Q]$, obtained without removing any of the protected elements at the beginning or the end.

Theorem 7.1.16. Let $\mathcal{U}_{k}$ be the class as defined in 7.1 .9 with the relation $\preceq$. Then, $\mathcal{U}_{k}$ is well-quasi-ordered under $\preceq$.

Proof. We prove this by induction on $k$. When $k=0$, the path presentations in the first component arise from when $P=Q$. Since $k=0$, we do not protect any elements, which implies $t=0$ and $b=0$. Thus the corresponding lattice path presentations can be represented by a 
combination of horizontal lines that go right or vertical lines that go up. Then these matroids correspond to nested matroids, which are well-quasi-ordered as mentioned above. Since $t=b=0, \mathcal{U}_{0}$ is also well-quasi-ordered.

Now, let $k$ be the smallest number such that $\mathcal{U}_{k}$ is not well-quasi-ordered. Then there exists a bad sequence in $\mathcal{U}_{k}$. Clearly, every bad sequence has only finitely many path presentations that belong to $\mathcal{U}_{k-1}$ as by the induction hypothesis, any infinite collection of $U_{k-1}$ cannot be an antichain. Thus we can safely remove this finite subsequence from the bad sequence without altering the property of being bad. Also, as there are only finitely many square presentations with square-width at most $k$, we only consider bad sequences that are made up entirely of triples that contain path presentations of square width $k$, that are not square presentations.

The size of a triple is the size of the corresponding presentation. We now construct a minimal bad sequence as follows: Assume we have chosen $\left(L_{1}, t_{1}, b_{1}\right), \ldots,\left(L_{i-1}, t_{i-1}, b_{i-1}\right)$ to be the initial segment of our minimal bad sequence. Then, $\left(L_{i}, t_{i}, b_{i}\right)$ is the next triple in the sequence if it is the smallest triple such that there exists a bad sequence that starts with $\left(L_{1}, t_{1}, b_{1}\right), \ldots,\left(L_{i-1}, t_{i-1}, b_{i-1}\right),\left(L_{i}, t_{i}, b_{i}\right)$. We denote this sequence by $U_{1}, U_{2}, U_{3}, \ldots$, where $U_{i}=\left(L_{i}, t_{i}, b_{i}\right)$. It can be seen easily that $U_{1}, U_{2}, U_{3}, \ldots$ is a bad sequence in itself. If not, there exists $i<j$ such that $U_{i} \preceq U_{j}$. By virtue of construction of the minimal bad sequence, $U_{j}$ is the smallest triple in the $j^{\text {th }}$ position among all bad sequences that start with $U_{1}, \ldots, U_{j-1}$. Thus $U_{i}$ is a minor of $U_{j}$, which contradicts the fact that $U_{1}, U_{2}, \ldots U_{j}$ appears at the beginning of a bad sequence. Each $U_{i}$ consists of a lattice path presentation that has a proper $k+1$ square because it is not a rectangular presentation. Say $L_{i}$ has a proper $k+1$ square at $j(i)$.

We apply the pulling apart operation as defined in Definition 7.1 .5 to the path presentations in the triple of the sequence $U_{1}, U_{2}, U_{3}, \ldots$ to obtain two new sequences $B_{1}, B_{2}, B_{3}, \ldots$, where $B_{i}=\left(B_{j(i)}\left(L_{i}\right), 1, b_{i}\right)$ and $T_{1}, T_{2}, T_{3}, \ldots$, where $T_{i}=\left(T_{j(i)}\left(L_{i}\right), t_{i}, 1\right)$. Since the sequence $U_{1}, U_{2}, U_{3}, \ldots$ is the minimal bad sequence, $B_{1}, B_{2}, B_{3}, \ldots$ cannot contain a bad subsequence. This can be seen as follows: let there exist a bad subsequence of $B_{1}, B_{2}, B_{3}, \ldots$, say $B_{i_{1}}, B_{i_{2}}, B_{i_{3}}, \ldots$, then $U_{1}, \ldots, U_{i_{1}-1}, B_{i_{1}}, B_{i_{2}}, \ldots$ is a bad 
sequence. If it were not a bad sequence, then there would exist $U_{k}$ and $B_{i j}$ such that $U_{k} \leq B_{i_{j}}$, for some $k \leq i_{1}-1$. We can easily observe from the proof of Lemma 7.1.6 that $B_{i} \leq U_{i}$, for any $i$. Hence, $U_{k} \leq B_{i_{j}} \leq U_{i_{j}}$. Now, $B_{i_{1}}$ is smaller than $U_{i_{1}}$ which contradicts the fact that $U_{1}, U_{2}, U_{3}, \ldots$ is the minimal bad sequence.

Thus in $B_{1}, B_{2}, B_{3}, \ldots$, there exists no bad subsequence. Hence by Lemma 7.1.13, there exists a subsequence $i_{1}<i_{2}<i_{3}<\ldots$ such that $B_{i_{1}} \leq$ $B_{i_{2}} \leq B_{i_{3}} \leq \ldots$. By the definition of bad sequences, for some $s<t$, $T_{i_{s}} \leq T_{i_{t}}$, or else $T_{i_{1}}, T_{i_{2}}, T_{i_{3}}, \ldots$ would be a bad sequence, which leads to a contradiction as before. Thus $B_{i_{s}} \leq B_{i_{t}}$ and $T_{i_{s}} \leq . T_{i_{t}}$. By Lemma 7.1.12. $G L\left(B_{i_{s}}, T_{i_{s}}\right)=U_{i_{s}}$ is a minor of $G L\left(B_{i_{t}}, T_{i_{t}}\right)=U_{i_{t}}$. This is a contradiction to our assumption that $U_{1}, U_{2}, U_{3}, \ldots$ is a bad sequence. Thus $\mathcal{U}_{k+1}$ is well-quasi-ordered.

Corollary 7.1.17. The class of lattice-path matroids of square-width at most $k$ is well-quasi-ordered.

Proof. Assume there is an infinite antichain of lattice-path matroids with square-width at most $k$. Let $M_{1}, M_{2}, M_{3}, \ldots$ be such an anti-chain where $\left[P_{i}, Q_{i}\right]$ is a corresponding lattice path presentation. But then $\left(M_{1}, 0,0\right),\left(M_{2}, 0,0\right),\left(M_{3}, 0,0\right), \ldots$ is an infinite anti-chain in $\mathcal{U}_{k}$. This contradicts Theorem 7.1.16 and thus $\left(M_{i}, 0,0\right) \leq\left(M_{j}, 0,0\right)$ for some $i<$ $j$, meaning there are disjoint subsets $I, J$ of $E\left(M_{j}\right)$ such that $M_{j} / I \backslash J$ is isomorphic to $M_{i}$. This is impossible since $M_{i}$ is not isomorphic to a minor of $M_{j}$.

Recall that we proved earlier in the section that a class of lattice path matroids has bounded branch-width if and only if it has bounded square-width. By proving the above result we have now proved Conjecture 1.1.1 for the class of lattice path matroids. 
64CHAPTER 7. ARE LATTICE PATH MATROIDS WELL-QUASI-ORDERED? 


\section{Chapter 8}

\section{Notch matroids and Well-Quasi-Ordering}

This chapter is the result of an intriguing question posed by Jim Geelen during the birthday conference of James Oxley held in Baton Rouge in July 2019. While presenting the results of the previous section, Jim was curious as to whether the anti-chain presented, square notches (defined below), is essentially the unique anti-chain in the class of lattice path matroids. We gave this further thought and had an enlightening discussion with Jim soon after. We answer the question in the affirmative in this section. We are indeed grateful to Jim Geelen for asking the right question as he always does!

Let $t \geq 3$ be an integer. We define a square notch matroid to be the matroid $M\left(N_{t}\right)$ where $N_{t}$ is the presentation

$\left[E^{(t-1)} N E N^{(t-1)}, N^{(t-1)} E N E^{(t-1)}\right]$. These matroids form a subclass of the notch matroids introduced in [5]. Figure 8.1 depicts the lattice path presentations $N_{3}, N_{4}$ and $N_{5}$.

We note that the class of square notch matroids forms an anti-chain in the class of lattice path matroids. This is justified by the observation that once we fix $k$, deletion/contraction of any element in $N_{k}$ destroys the 'notch' at the top/bottom that is characteristic of the presentations in this class. We prove that square notches essentially form the only anti-chain in the class 

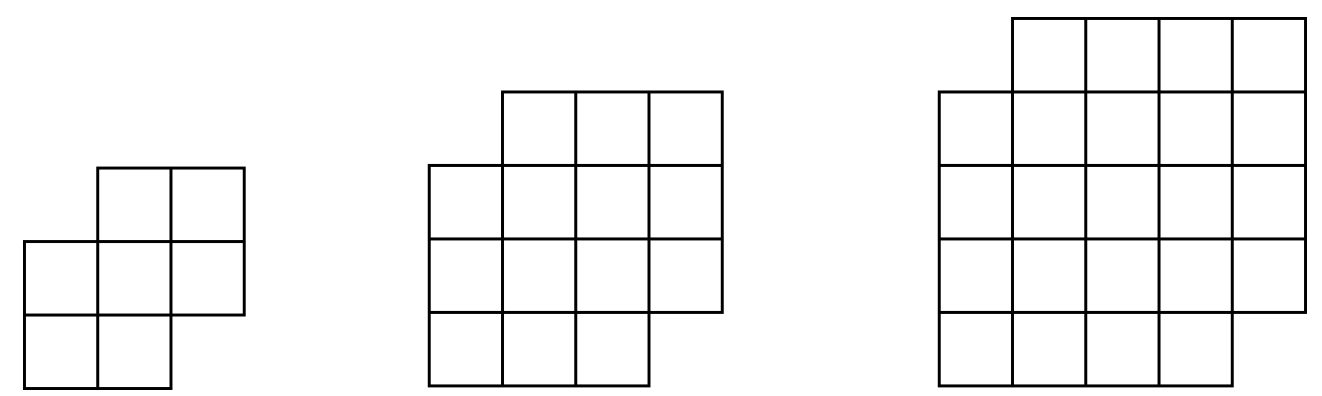

Figure 8.1: $N_{3}, N_{4}$ and $N_{5}$ respectively

of lattice path matroids. We state this theorem below:

Theorem 8.1.1. Let $M$ be a minor-closed class of lattice path matroids that contains only finitely many square notch matroids. Then $M$ is well-quasi-ordered.

We need a few definitions before we can prove the above statement.

Definition 8.1.2. Let $[P, Q]$ be a path presentation. Then notch-width of $[P, Q]$ is said to be the largest $k$ such that $N_{k}$ is a minor of $[P, Q]$. If no such minor exists, then we define the notch-width to be 0 .

Definition 8.1.3. Let $k$ be a non-negative integer. Then, we let $\mathcal{N}_{k}$ be the set of lattice triples $([P, Q], t, b)$, where $[P, Q]$ is a lattice-path presentation with notch-width at most $k$, and $t, b$ are either 0 or 1 . When $t=1$, the last $k$ elements of $L$ are being protected and when $t=0$, none of the last $k$ elements are protected. Similarly, when $b=1$, the first $k$ elements are protected, and when $b=0$, none of these elements are being protected.

Let $\left(\left[P^{\prime}, Q^{\prime}\right], t^{\prime}, b^{\prime}\right),([P, Q], t, b) \in \mathcal{N}_{k}$. We say that $\left(\left[P^{\prime}, Q^{\prime}\right], t^{\prime}, b^{\prime}\right) \preceq$ $([P, Q], t, b)$, that is, $\left(\left[P^{\prime}, Q^{\prime}\right], t^{\prime}, b^{\prime}\right)$ is a triple minor of $([P, Q], t, b)$, if there exists disjoint subsets $I, J$ of $\{1, \ldots, m+r\}$ such that $[P, Q] / I \backslash J=\left[P^{\prime}, Q^{\prime}\right]$, and if $t=1$ then $I \cup J$ has no element in $\{m+r-k+1, \ldots, m+r-1, m+r\}$, and if $b=1$, then $I \cup J$ has no element in $\{1, \ldots, k\}$.

We now prove a lemma regarding squares in a lattice path matroid that will prove beneficial for the proof of our main result. Note that $[P, Q]$ has a $k \times k$ square at $i$ if and only if the dual of $[P, Q]$ does.

Lemma 8.1.4. Assume that $[P, Q]$ is not a rectangle, and that $[P, Q]$ has a minor with a proper $k \times k$ square. Then $[P, Q]$ has a proper $k \times k$ square. 
Proof. Let $[P, Q]$ be a smallest counterexample, so $m+r$ is as small as possible with respect to the lemma failing.

Up to duality, we can assume that $[P, Q] \backslash e$ has a minor with a proper $k \times$ $k$ square. By minimality, $[P, Q] \backslash e$ has a proper $k \times k$ square and let this proper $k \times k$ square be at $i$.

We can recover $[P, Q]$ from $[P, Q] \backslash e$ by inserting a copy of $E$ into $P$ before, say position $p$, and inserting $E$ into $Q$ after, say position $q$, where $p \leq$ $q+1$. Furthermore, any character in $[P, Q] \backslash e$ in either the top or bottom path in position greater than or equal to $p$ but no more than $q$ must be $N$. After these two insertions, we can recover $[P, Q] \backslash e$ by deleting any position between the two new copies of $E$, including the positions where they have been inserted.

If $i \leq p$ then $[P, Q]$ has a proper $k \times k$ square at $i$. If $i \geq q$, then $[P, Q]$ has a proper $k \times k$ square at $i+1$.

Now we assume $p<i<q$. Because there are only copies of $N$ between $p$ and $i$, and between $i$ and $q,[P, Q] \backslash e$ has a $k \times k$ square at $p-1$, and also at $q$. If either of these is proper, then we are done by the previous paragraph. So now we assume that $p-1=k$, and $k=m+r-q$. This implies that $P=E^{(k+1)} N^{(m+r-k-1)}$ and $Q=N^{(m+r-k-1)} E^{(k+1)}$, which is a contradiction, as $[P, Q]$ is not a rectangle.

Theorem 8.1.5. Let $\mathcal{N}_{k}$ be as defined in 8.1.3 with the relation $\preceq$. Then $\mathcal{N}_{k}$ is well-quasi-ordered.

Proof. Assume that the theorem fails for $k$, where $k$ is as small as possible.

By Lemma 7.1.15, any bad sequence in $\mathcal{N}_{k}$ can only contain finitely many triples of the form $([P, Q], t, b)$ where $M[P, Q]$ is a nested matroid. Hence, we consider only the bad sequences in $\mathcal{N}_{k}$ where every triple in the bad sequence contains $\left(N_{t}, 0,0\right)$ as a minor for some $t \geq 3$. We do so as any non-nested matroid contains a square notch [29].

Assume that a bad sequence in $\mathcal{N}_{k}$ contains infinitely many triples that do not have $\left(N_{k}, 0,0\right)$ as a minor in the order $\preceq$. Then all such triples are in $\mathcal{N}_{k-1}$ and they form an anti-chain in $\mathcal{N}_{k-1}$. But this contradicts our choice of $k$. So any bad sequence of $\mathcal{N}_{k}$ can be assumed to contain only triples that have notch width $k$. 
Now, we construct a minimal bad sequence as was done in the proof of Theorem 7.1.16. Let it be $L_{1}, L_{2}, L_{3}, \ldots$ where each $L_{j}=\left(\left[P_{j}, Q_{j}\right], t_{j}, b_{j}\right)$. Because every presentation in a triple in the bad sequence has notch width $k$, it has an $N_{k}$ minor. Then, since $N_{k}$ has a proper $(k-2) \times$ $(k-2)$ square, by Lemma 8.1.4, every presentation has a $(k-2) \times$ $(k-2)$ square, say at $j(i)$. Let $B_{1}, B_{2}, B_{3}, \ldots$ be a sequence where each $B_{j}=\left(\left[B_{i(j)}(P), B_{i(j)}(Q)\right], 1, b_{j}\right)$ and $T_{1}, T_{2}, T_{3}, \ldots$ be a sequence where each $T_{j}=\left(\left[T_{i(j)}(P), T_{i(j)}(Q)\right], t_{j}, 1\right)$ and $\left[B_{i(j)}(P), B_{i(j)}(Q)\right],\left[T_{i(j)}(P), T_{i(j)}(Q)\right]$ are as in Definition 7.1.5. Since the sequence $L_{1}, L_{2}, L_{3}, \ldots$ is a minimal bad sequence, $B_{1}, B_{2}, B_{3}, \ldots$ cannot contain a bad subsequence.

Hence by Lemma 7.1.13, there exists a subsequence $i_{1}<i_{2}<i_{3}<\ldots$ such that $B_{i_{1}} \preceq B_{i_{2}} \preceq B_{i_{3}} \preceq \ldots$. By the definition of bad sequences, for some $s<t, T_{i_{s}} \preceq T_{i_{t}}$, or else $T_{i_{1}}, T_{i_{2}}, T_{i_{3}}, \ldots$ would be a bad sequence, which leads to a contradiction as before. Thus $B_{i_{s}} \preceq B_{i_{t}}$ and $T_{i_{s}} \preceq T_{i_{t}}$. By Lemma 7.1.12, $G L\left(B_{i_{s}}, T_{i_{s}}\right)=L_{i_{s}}$ is a minor of $G L\left(B_{i_{t}}, T_{i_{t}}\right)=L_{i_{t}}$. This is a contradiction to our assumption that $L_{1}, L_{2}, L_{3}, \ldots$ is a bad sequence. Thus $\mathcal{N}_{k}$ is well-quasi-ordered.

Corollary 8.1.6. Any minor-closed family of lattice-path matroids that has bounded notch-width is well-quasi-ordered.

Proof. Assume there is an infinite antichain of lattice-path matroids with notch-width at most $k$. Let $M_{1}, M_{2}, M_{3}, \ldots$ be such an anti-chain where $\left[P_{i}, Q_{i}\right]$ is a corresponding lattice path presentation. But then $\left(M_{1}, 0,0\right),\left(M_{2}, 0,0\right),\left(M_{3}, 0,0\right), \ldots$ is an infinite anti-chain in $\mathcal{N}_{k}$. For otherwise, $\left(M_{i}, 0,0\right) \leq\left(M_{j}, 0,0\right)$ for some $i<j$, meaning there are disjoint subsets $I, J$ of $E\left(M_{j}\right)$ such that $M_{j} / I \backslash J$ is isomorphic to $M_{i}$. This is impossible since $M_{i}$ is not isomorphic to a minor of $M_{j}$.

This proves that the class of square notch-matroids are to be 'avoided' in well-quasi-ordered classes of lattice path matroids. Hence we have the following result:

Corollary 8.1.7. A minor-closed class of lattice-path matroids is well-quasi-ordered if and only if it contains only finitely many square notch matroids. 


\section{Part III}

\section{Fundamental and bicircular matroids}



This part focuses on fundamental transversal matroids and bicircular matroids. Even though the class of fundamental matroids is not closed under minors, it lies in the intersection of transversal and cotransversal and this makes the class worthy of further investigation. The fact that it has an intuitive geometric representation only adds to its appeal. The first chapter in this part explores the question of recognising fundamental matroids in the class of transversal matroids. The same exercise is repeated in Chapters 10 and 11 in the classes of lattice-path matroids and bicircular matroids respectively.

In the last chapter, we examine the intersection of the classes of bicircular and cobicircular matroids. We define whirly-swirls and conjecture that for large enough matroids, these are the only members in this intersection. 


\section{Chapter 9}

\section{The problem FUNDAMENTAL TRANSVERSAL MATROID}

In this chapter we explore the class of fundamental transversal matroids. We aim to recognize when a given transversal matroid is fundamental. Even though it is quite challenging in general, we strive to do so for special sub-classes. We also investigate the complexity of this question in general. Consider the problem FUNDAMENTAL TRANSVERSAL MATROID as follows:

\section{FUNDAMENTAL TRANSVERSAL MATROID}

INPUT: A transversal matroid $M$ represented by a bipartite graph

QUESTION: Is $M$ fundamental?

Recall that by Theorem 2.1.2, every transversal matroid $M$ has a representation on a simplex with $\operatorname{rank}(M)$ vertices and every cyclic flat $F$ can be represented as a $\operatorname{rank}(F)$-vertex face of this simplex. By the definition of a fundamental basis, the elements on the vertices of this simplex form a fundamental basis $X$. If we are given a geometric representation in which there is an element on each vertex of the simplex, then this is easy to recognise, but there may be many possible geometric representations as the simplex representation for a transversal matroid is not unique. This poses a difficulty in trying to identify fundamental 
matroids in general. We first prove a lemma that will help in classifying this problem as NP.

Lemma 9.1.1. Let $G$ be a bipartite presentation of the transversal matroid $M$. Let $F$ be a flat in $M$ and let $e$ be an element in $F$. Let $v_{1}, \ldots, v_{t}$ be the neighbours of vertices in $F$. Then e is not freely placed in $F$ if and only if there exists a vertex $v_{i}$ such that $e$ is contained in a circuit in the transversal matroid represented by the bipartite graph $G_{i}=G-\left(v_{i} \cup N\left(v_{i}\right)\right)$.

Proof. Let there exist a vertex $v_{i}$ such that $e$ is contained in a circuit $C$ in the transversal matroid, $N$, represented by the bipartite graph $G_{i}$. We claim that $\mathrm{cl}_{N}(C)=\mathrm{cl}_{M}(C)$. If not, then there exists $f \in \mathrm{cl}_{M}(C)-\mathrm{cl}_{N}(C)$. Clearly, $f \in E(M)-E(N)$. Also, $f$ is adjacent to $v_{i}$ which implies that $\mathrm{r}_{M}(C \cup f)=\mathrm{r}_{M}(C)+1$ as no element of $C$ is adjacent to $v_{i}$. This contradicts the fact that $f \in \mathrm{cl}_{M}(C)-\mathrm{cl}_{N}(C)$. Hence, $\mathrm{cl}_{M}(C)=\mathrm{cl}_{N}(C)$. Thus $\operatorname{cl}(C)$ does not contain $F$ but contains $e$, which implies that $e$ is not free.

Now, let $e$ not be freely placed in $F$. That is, there exists a cyclic flat $Z$ that contains $e$ but not $F$. Then, there exist $C \subseteq F$ such that $e \in C$ but $\operatorname{cl}(C)$ does not contain $F$. Since $C-e$ is independent, it can be matched in $G$, so $C$ has at least $|C-e|$ neighbours in $G$. But $C$ is dependent, so Hall's Theorem [16] tells us that some subset of $C$ has fewer neighbours than its cardinality. This subset can only be $C$. Hence, $C$ has exactly $|C|-1$ neighbours. If every vertex in $v_{1}, \ldots, v_{t}$ is a neighbour of some vertex in $C$, then $F$ is in the closure of $C$, a contradiction. Hence there is some $v_{k}$ that is not a neighbour of a vertex in $C$. Hence in the bipartite graph $G_{k}, e$ is contained in $C$. Thus proved.

If we combine the above lemma and Lemma 2.2.4, then it becomes possible to efficiently check whether $B$ is a fundamental basis.

Theorem 9.1.2. The problem FUNDAMENTAL TRANSVERSAL MATROID is in NP.

Proof. Let $M$ be a transversal matroid. The certificate that helps us is a basis $B$ that is fundamental. By Lemma 2.2.4, we are only required to check that every element $e$ that is not in $B$ is free in its fundamental circuit $C(e, B)$. 
We can find the fundamental circuit $C(e, B)$ by proceeding through every element $x$ in $M$ and using a matching algorithm to test whether $(B-x) \cup e$ is a basis. This can be done in polynomial time. Then, by Lemma 9.1.1, this implies that we find every neighbour of $e$ in $G$ and check whether in every graph $G_{i}$ (as described in the statement of the lemma), $e$ is a coloop, which can again be achieved in polynomial time. If this turns out to be true, then $M$ is fundamental.

The following theorem [2] is a characterisation of fundamental matroids. Since, this characterisation involves checking every subfamily of cyclic flats of a matroid, we cannot expect it to lead to a polynomial time algorithm.

Theorem 9.1.3. A transversal matroid $M$ is fundamental if and only if for all non-empty subsets $\mathcal{F}$ of $\mathcal{Z}(M)$, the collection of all cyclic flats of $M$, the following holds true:

$$
r(\cap \mathcal{F})=\sum_{\mathcal{F}^{\prime} \subseteq \mathcal{F}}(-1)^{\left|\mathcal{F}^{\prime}\right|+1} r\left(\cup \mathcal{F}^{\prime}\right)
$$

Thus we end with the following conjecture:

Conjecture 9.1.4. The problem FUNDAMENTAL TRANSVERSAL MATROID is NP-complete. 
76CHAPTER 9. THE PROBLEM FUNDAMENTAL TRANSVERSAL MATROID 


\section{Chapter 10}

\section{Recognising fundamental lattice path matroids}

If a transversal matroid $M$ is not fundamental, then it does not have a fundamental basis. In other words, for every basis $X$, there exists a cyclic flat $F$ such that $F \cap X$ does not span $F$. Finding out whether a fundamental basis exists is not an easy task in the case of a general transversal matroid, as we saw above. But the elegant structural properties that lattice path matroids possess, makes the hunt for a fundamental basis relatively simpler and much more fun in this class!

Let $M$ be a rank- $r$ lattice path matroid on the set $[1, n]$, where $N_{1}, \ldots, N_{r}$ are intervals in $[1, n]$ such that each $N_{i}=\left[l_{i}, u_{i}\right]$, where $l_{1}<\cdots<l_{r}$ and $u_{1}<\cdots<u_{r}$. Let $E=[1, n]$ and $B=\left\{N_{1}, \ldots, N_{r}\right\}$. We can then identify the sets $E$ and $B$ with the partition of the set of vertices of a bipartite graph $G(E, B)$, where $i$ is adjacent to $N_{j}$ if and only if $i \in N_{j}$. Hence, $M$ is the transversal matroid on ground set $E$. Figure 10.1 is an example of a lattice path matroid that is fundamental transversal and Figure 10.2 gives an example of one that is not fundamental transversal.
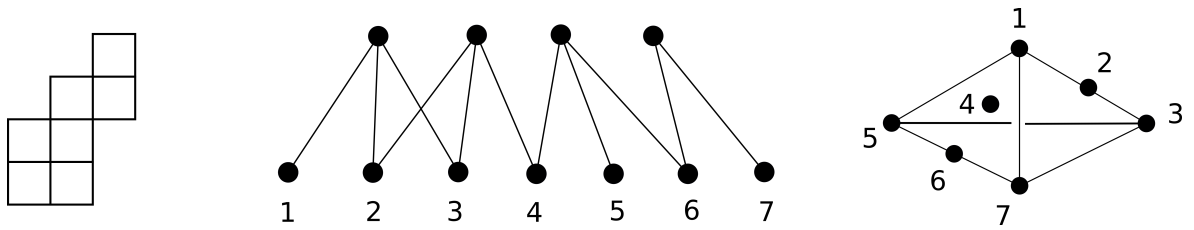

Figure 10.1: A lattice path matroid that is also fundamental transversal 

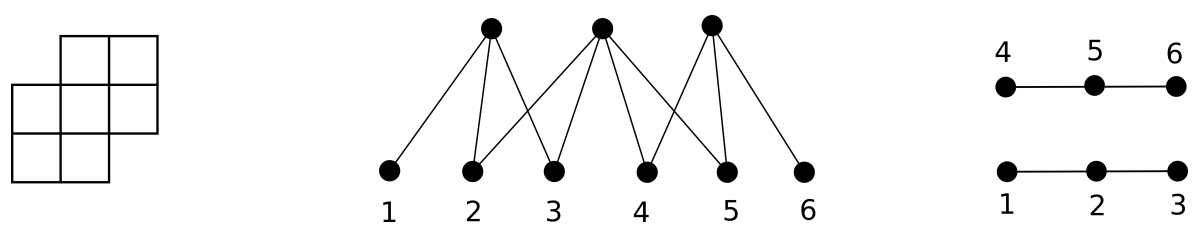

Figure 10.2: A lattice path matroid that is not fundamental transversal

A basis, say $X$, of $M$ is a subset $i_{1}, \ldots, i_{r}$ of $[1, n]$, such that $i_{1}<\cdots<i_{r}$ and $i_{j} \in N_{j}$, for every $j$.

The class $\mathcal{L}$, of lattice path matroids, $\mathcal{L}$ is closed under minors, whereas the class of fundamental transversal matroids is not closed under deletion or contraction. Also note that neither class is contained in the other. We have seen above in Figure 10.2 an example of a lattice path matroid that is not fundamental transversal. The 3-whirl, $\mathcal{W}^{3}$, is an excluded minor for $\mathcal{L}$ but is a fundamental transversal matroid as seen below [1].
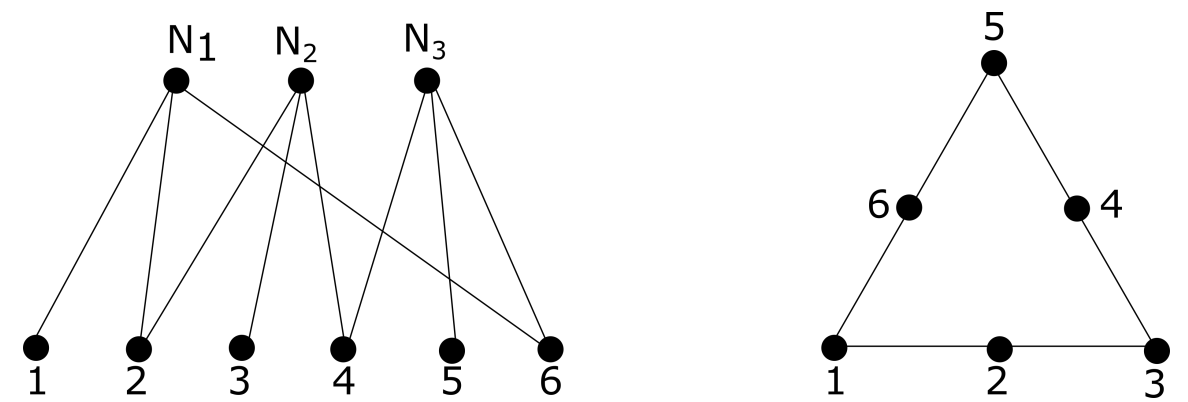

Figure 10.3: The 3-whirl as represented in a bipartite graph and on a simplex

It is well-known that every cyclic flat of a transversal matroid has the form $E-N(Z)$, where $Z \subseteq B$ and $N(Z)$ is the set of vertices adjacent to some vertex in $Z$. Note that not every set of the form $E-N(Z)$ is cyclic. For example, consider the matroid represented by the bipartite graph in Figure 10.4. If $Z$ is the set of vertices in the box, then $E-N(Z)$ is not cyclic as 1 and 2 are both co-loops in $M \mid\{1,2,6,7\}$. 


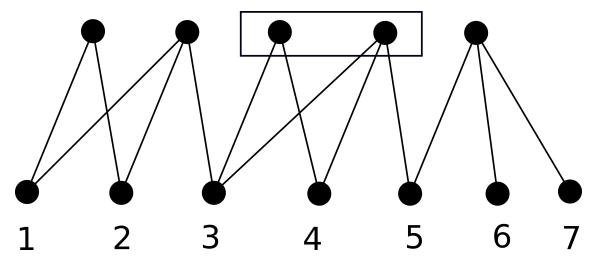

Figure 10.4: If $Z$ is the set of vertices in the box, then $E-N(Z)$ is not cyclic

In a transversal matroid, the following theorem illustrates what a degree one vertex in $B$ signifies. Note that if $N_{i}=\{x\}$, then the bounding paths $P$ and $Q$ contain a common vertical edge in the lattice path presentation.

Proposition 10.1.1. If $N_{i}=\{x\}$, then $x$ corresponds to a coloop in $M$.

Proof. Let $X$ be a maximum matching, which corresponds to a basis of $M$. If the neighbour of $N_{i}$, say $x$, is not matched in $X$, then adding the edge $\left\{N_{i}, x\right\}$ to the matching yields a bigger matching than $X$. This is a contradiction to the fact that $X$ is maximum, and hence $x$ is in every basis.

In general, a coloop in a transversal matroid need not be a degree-one vertex. For example, consider $K_{n, n}$, a bipartite presentation for $U_{n, n}$. Every element is a coloop in $U_{n, n}$ but every vertex in $K_{n, n}$ has degree $n$. On the other hand, for lattice path matroids we have the following result that makes it easier to identify coloops, and hence cyclic flats from the structure of the bipartite presentation.

Proposition 10.1.2. In a lattice path matroid, any coloop corresponds to a degree-one vertex in $B$.

Proof. Let $x$ in $E$ be such that $x$ is a coloop of $M$. Note that $x$ is in every basis $X$ of $M$. This implies that, in the corresponding lattice path presentation, we are forced to go North at step $x$. If after traversing $x-1$ steps, we have two possible arrival points, then from the left most destination we can travel East for step $x$. But this cannot be the case as $x$ is a coloop. Hence, we arrive at a unique point after $x-1$ steps and the next step possible is only a North step. Then, in the bipartite presentation this corresponds to a degree one vertex in $B$ which has an edge incident to $x$. 
When $x=1$ is a coloop of $M$, the first step has to be a North step, and this again corresponds to $N_{1}$ having degree-one in $G(E, B)$. Hence proved.

Corollary 10.1.3. In a lattice path matroid $M$, if $Z$ is a subset of $B$, then $E-$ $N(Z)$ is cyclic if and only if there exists no degree-one vertex in $B$ in the subgraph $G(E, B)-(Z \cup N(Z))$.

Proof. Note that, deleting $N(Z)$ from a lattice presentation yields another lattice path presentation. In a lattice path matroid $M, E-N(Z)$ is cyclic if and only if there exists no coloop in the matroid $M \mid(E-N(Z))$. By Propositions 10.1.1 and 10.1.2 this is true if and only if there exists no degree-one vertex in $B$ after deleting $Z$.

The above results specific to lattice path matroids help us develop the following definitions that will later prove useful in defining fundamental lattice-path matroids.

Definition 10.1.4. A subset $Z$ of $B$ is said to be cyclic if $E-N(Z)$ is a cyclic flat and $B$ has no isolated vertices in $G(E, B)-(Z \cup N(Z))$.

Definition 10.1.5. Let $X$ be a basis of $M$. A cyclic subset $Z$ of $B$ is said to be good (with respect to $X$ ) if $X-N(Z)$ spans $E-N(Z)$ in $M$. Otherwise $Z$ is bad.

Note that a basis is fundamental if and only if every cyclic subset is good relative to it.

Remark 10.1.6. If $Z$ is cyclic and $X$ is a basis, then $N(Z)$ contains at least $|Z|$ elements that are also in $X$. If it contains exactly that, then it is good, else it is bad. Note that in a bipartite presentation of a lattice path matroid, any maximum matching will saturate $B$.

Proposition 10.1.7. If $Z$ is any good cyclic subset of $B$ (with respect to $X$ ), then $X \cap N(Z)$ spans $N(Z)$.

Proof. Since $X$ is a basis of $M$, it must be matched to $B$. So the number of vertices in $X$ and $N(Z)$ is at least $|Z|$. But, this is also an upper bound on the rank of $N(Z)$. 
Definition 10.1.8. A segment in a sequence of subsets $A_{1}, A_{2}, \ldots, A_{k}$ is defined to be a subsequence of subsets $A_{i}, \ldots, A_{i+j}$ for some non-negative integer $j$, where $1 \leq i \leq k$ and $i+j \leq k$.

Note that $B=N_{1}, \ldots, N_{r}$ and hence a segment of $B$ is of the form $N_{i}, \ldots, N_{i+j}$.

Lemma 10.1.9. If $B$ has a bad subset $Z$ (with respect to $X$ ), then $Z$ has a bad subset that is a segment in $N_{1}, \ldots, N_{r}$.

Proof. Let $Z$ be a bad subset of $B$. We will prove by an induction argument that $Z$ has a bad segment. Note that $Z$ can certainly be considered to be a union of segments. Assume the lemma fails for $Z$, where $Z$ is chosen to have the smallest number of segments.

Note that $Z$ has at least two segments. Let $\left(Z_{L}, Z_{R}\right)$ be a partition of $Z$ such that there exists $k$ with the property that $N_{k} \in Z$ and if $N_{i} \in Z$, then $i \leq k$ implies that $N_{i} \in Z_{L}$, else $N_{i} \in Z_{R}$. In particular $N_{k+1}$ is not in $Z$. Then there exists a vertex in $B$ that lies in between $Z_{L}$ and $Z_{R}$. If $Z_{L}$ and $Z_{R}$ have common neighbours, then every vertex in $B$ that lies between $Z_{L}$ and $Z_{R}$ has degree 0 in $G-(Z \cup N(Z))$, which implies that $Z$ is not cyclic. Hence $Z_{L}$ and $Z_{R}$ have no common neighbours. But, if the set of neighbours of $Z_{L}$ and $Z_{R}$ have no elements in common, then $Z_{L}$ and $Z_{R}$ are both cyclic, unless the last neighbour of $Z_{L}$ is exactly one less than the first neighbour of $Z_{R}$. In this case, every $z \in B$ that is in between $Z_{L}$ and $Z_{R}$, has degree zero after deleting $N(Z)$. This is again impossible by the previous argument, as it would imply that $Z$ is not cyclic.

Assume that neither of $Z_{L}$ and $Z_{R}$ are bad subsets of $B$. Then, $X-N\left(Z_{L}\right)$ and $X-N\left(Z_{R}\right)$ span $E-N\left(Z_{L}\right)$ and $E-N\left(Z_{R}\right)$ respectively. Thus, $r(E-$ $\left.N\left(Z_{L}\right)\right)=\left|X \cap\left(E-N\left(Z_{L}\right)\right)\right|$ and $r\left(E-N\left(Z_{R}\right)\right)=\left|X \cap\left(E-N\left(Z_{R}\right)\right)\right|$. Hence, $r\left(N\left(Z_{L}\right)\right)=\left|Z_{L}\right|$ and $r\left(N\left(Z_{R}\right)\right)=\left|Z_{R}\right|$, as $\left|X \cap Z_{L}\right|=\left|Z_{L}\right|$ and $X$ is independent. Then, $r(N(Z))=r\left(N\left(Z_{L} \cup Z_{R}\right)\right) \leq\left|Z_{L}\right|+\left|Z_{R}\right|=|Z|$, which is a contradiction, as this implies that $r(N(Z))=|Z|$ which makes $Z$ good. Hence, one of $Z_{L}$ or $Z_{R}$ is a bad subset. By induction, this implies that either $Z_{L}$ or $Z_{R}$ has a bad segment. Hence proved. 
Definition 10.1.10. A basis $X$ of $E$, where $|X|=r$ is defined to be good going forwards if for every $Z \subseteq B$, such that $Z$ is cyclic and $Z=\left\{N_{1}, \ldots, N_{i}\right\}$, for some $1<i<r, Z$ is good relative to $X$.

A subset $X$ of $E$, where $|X|=r$ is defined to be good going backwards if for every $Z \subseteq B$, such that $Z$ is cyclic and $Z=\left\{N_{i}, \ldots, N_{r}\right\}$, for some $1<i<r$, $Z$ is good.

Lemma 10.1.11. If $X$ is a basis that is good going backwards and forwards, then $\left\{N_{1}, \ldots, N_{r}\right\}$ has no bad subsets (with respect to $X$ ).

Proof. By Lemma 10.1.9, if there exists a bad subset, then there exists a bad segment. Let $Z=N_{i}, \ldots, N_{j}$ be a bad segment of $B$. Then $Z_{1}=N_{1}, \ldots, N_{j}$ and $Z_{2}=N_{i}, \ldots, N_{r}$ are also cyclic subsets of $B$. But, since $X$ is good going backwards and forwards, $X \cap N\left(Z_{1}\right)$ and $X \cap N\left(Z_{2}\right)$ span $N\left(Z_{1}\right)$ and $N\left(Z_{2}\right)$ respectively. Thus $\left|X \cap N\left(Z_{1}\right)\right|=\left|Z_{1}\right|$ and $\left|X \cap N\left(Z_{2}\right)\right|=\left|Z_{2}\right|$. Hence, $|X \cap Z|=\left|X \cap\left(Z_{1} \cap Z_{2}\right)\right|=\left|X \cap N\left(Z_{1}\right)\right|+\left|X \cap N\left(Z_{2}\right)\right|-\mid X \cap$ $\left(N\left(Z_{1}\right) \cup N\left(Z_{2}\right)\right)|=| Z_{1}|+| Z_{2}|-| X|=| B|+| Z|-| B|=| Z \mid$. This implies that $X-N(Z)$ spans $E-N(Z)$, which is a contradiction to the fact that $Z$ is bad.

With the aid of the above results, we are now ready to devise an algorithm that can check whether a given lattice path matroid is fundamental transversal. This polynomial time algorithm runs as follows:

Algorithm: FUNDAMENTAL LATTICE PATH MATROID

Input: A bipartite presentation corresponding to the lattice path matroid M

Output: A fundamental basis $X$, if such a basis exists

(1) Let $Z^{F_{i}}$ be the smallest cyclic forward segment of the form $\left\{N_{1}, \ldots, N_{j}\right\}$ that contains $N_{1} \cup \ldots \cup N_{i}$. Similarly, let $Z^{B_{i}}$ be the smallest cyclic backward segment of the form $\left\{N_{j}, \ldots, N_{r}\right\}$ that contains $N_{i} \cup \ldots \cup N_{r}$. This is easy to find due to Corollary 10.1.3.

(2) Let $F_{1}=N\left(Z^{F_{1}}\right)$ and for $i>1, F_{i}=N\left(Z^{F_{i}}\right)-N\left(Z^{F_{i-a}}\right)$, where $a$ is the smallest positive integer such that $Z^{F_{i-a}} \neq Z^{F_{i}}$. If no such $a$ exists, then $F_{i}=F_{1}$. Similarly, $B_{r}=N\left(Z^{B_{r}}\right)$ and for $i<r, B_{i}=$ 
$N\left(Z^{B_{i}}\right)-N\left(Z^{B_{i+a}}\right)$, where $a$ is the smallest positive integer such that $Z^{B_{i+a}} \neq Z^{B_{i}}$. If no such $a$ exists, then $B_{i}=B_{r}$.

(3) Initialise $X$ to be empty. For each $i$, choose $x_{i}$ from the set $\left(N_{i} \cap F_{i} \cap\right.$ $\left.B_{i}\right)-X$. (Note that there might be more than one choice for $x_{i}$ and in this case we choose the first one in the set.) If at any stage $x_{i}$ does not exist, then terminate the algorithm. Else, output $X$.

(4) If the algorithm terminates without an output, then $M$ is not a fundamental transversal matroid. Else, $X$ is a fundamental basis of $M$ and hence $M$ is fundamental.

Theorem 10.1.12. There exists a polynomial time algorithm to check whether a lattice path matroid is fundamental, when the matroid is input as the corresponding bipartite graph.

Proof. Consider the algorithm FUNDAMENTAL LATTICE PATH MATROID and let $M$ be a lattice path matroid. We prove that a lattice path matroid is fundamental if and only if we can find a basis that satisfies the algorithm.

By Lemma 10.1.11, finding a fundamental basis boils down to finding a basis that is good going backwards and forwards. Assume the algorithm finds a set $X$. Note that if the algorithm finds such a set, then each $x_{i} \in X$ is in $N_{i}$, by construction, so $X$ is indeed a basis. If $X$ is not a fundamental basis, then by Lemma 10.1.11 there is a cyclic set $Z$ that is either bad going backwards or forwards. Assume $Z=Z^{F_{i}}$ is bad with $Z^{F_{i}}=N_{1}, \ldots, N_{i}$. (If $Z^{F_{i}} \neq N_{1}, \ldots, N_{i}$, then we keep going 'forwards' to find an $i$ where this is true.) Note that $Z^{F_{i}}$ contains elements $x_{1}, \ldots, x_{i}$ by virtue of construction. Now, as it is also bad, $N\left(Z^{F_{i}}\right)$ contains more than $i$ elements of $X$ and hence contains $x_{j}$ where $j>i$ and $x_{j} \in\left(N_{j} \cap F_{j} \cap B_{j}\right)$. If $Z^{F_{i}} \neq Z^{F_{j}}$, then there exists $i \leq i^{\prime}<j$ such that $F_{j}=N\left(Z^{F_{j}}\right)-N\left(Z^{F_{i}^{\prime}}\right)$. But this implies that $N\left(Z^{F_{i}^{\prime}}\right)$ contains $N\left(Z^{F_{i}}\right)$ which contains $x_{j}$. This leads to a contradiction as $x_{j}$ is in $F_{j}$ and $Z^{F_{i}^{\prime}}$. Thus $Z^{F_{i}}=Z^{F_{j}}$ which leads to another contradiction as $Z^{F_{i}}=N_{1}, \ldots, N_{i}$ and does not contain $N_{j}$ which is in $Z^{F_{j}}$.

Similarly, if $Z$ is $Z^{B_{i}}=N_{i}, \ldots, N_{r}$, then there exists $j<i$ such that $Z^{B_{i}}$ contains $x_{j} \in\left(N_{j} \cap F_{j} \cap B_{j}\right)$. If $Z^{B_{i}} \neq Z^{B_{j}}$, then there exists $i^{\prime}$ such that 
$j<i^{\prime} \leq i$ and $B_{j}=Z^{B_{j}}-Z^{B_{i^{\prime}}}$. Thus, $x_{j}$ is in $N\left(Z^{B_{i}^{\prime}}\right)$ as it contains $N\left(Z^{B_{i}}\right)$ and in $B_{j}$, which is a contradiction. Hence, $Z^{B_{i}}=Z^{B_{j}}$, which leads to a contradiction as above. Therefore, whenever the algorithm returns a set, it is a fundamental basis.

For the converse, assume that $M$ is a fundamental lattice path matroid. Then, there exists a fundamental basis $X$ and let $x_{i}$ be an element in $X \cap N_{i}$ for every $i$. We claim that the algorithm can find $x_{i}$ for every $i$. Assume this does not hold true and let $i$ be the first integer for which this fails, that is, the algorithm finds $x_{1}, \ldots, x_{i-1}$ but not $x_{i}$. Then $x_{i}$ is either not in $F_{i}$ or not in $B_{i}$. If it is the former case, then since $x_{i} \in N_{i}$, it is also in $N\left(Z^{F_{i}}\right)$. Thus, $x_{i} \in Z_{i-a^{\prime}}^{F}$ where $a$ is the smallest positive integer such that $Z^{F_{i-a}} \neq Z^{F_{i}}$. The minimality of $a$ implies that $Z^{F_{i-a}}=N_{1}, \ldots, N_{i-a}$ and $Z^{F_{i-a}}$ is a cyclic set. Then $Z^{F_{i-a}}$ contains $x_{1}, \ldots, x_{i-a}$ and $x_{i}$. But this implies that $Z^{F_{i-a}}$ is a bad cyclic set contradicting the fact that $X$ is a fundamental basis. Similarly assume that $x_{i}$ is not in $B_{i}$. Then, since $x \in N_{i}, x \in N\left(Z^{B_{i}}\right)$ and $x_{i} \in Z_{i+a}^{B}$, where $a$ is the smallest positive integer such that $Z^{B_{i+a}} \neq Z^{B_{i}}$. Again, the minimality of $a$ implies that $Z^{F_{i+a}}=N_{i+a}, \ldots, N_{r}$ and $Z^{B_{i+a}}$ is a cyclic set. Then $Z^{B_{i+a}}$ contains $x_{i+a}, \ldots, x_{r}$ and $x_{i}$. But this implies that $Z^{B_{i+a}}$ is a bad cyclic set contradicting the fact that $X$ is a fundamental basis. Hence, the algorithm returns a fundamental basis if there exists one.

We first illustrate how the algorithm works for a lattice path matroid $M_{1}$ that is also fundamental (See Figure 10.5). Here, $E=[1,7]$ and $B=\{[1,2],[2,4],[3,5],[5,7]\}$. For the sake of brevity, we denote $N_{1}, \ldots, N_{i}$ as $N_{1 \ldots i}$.
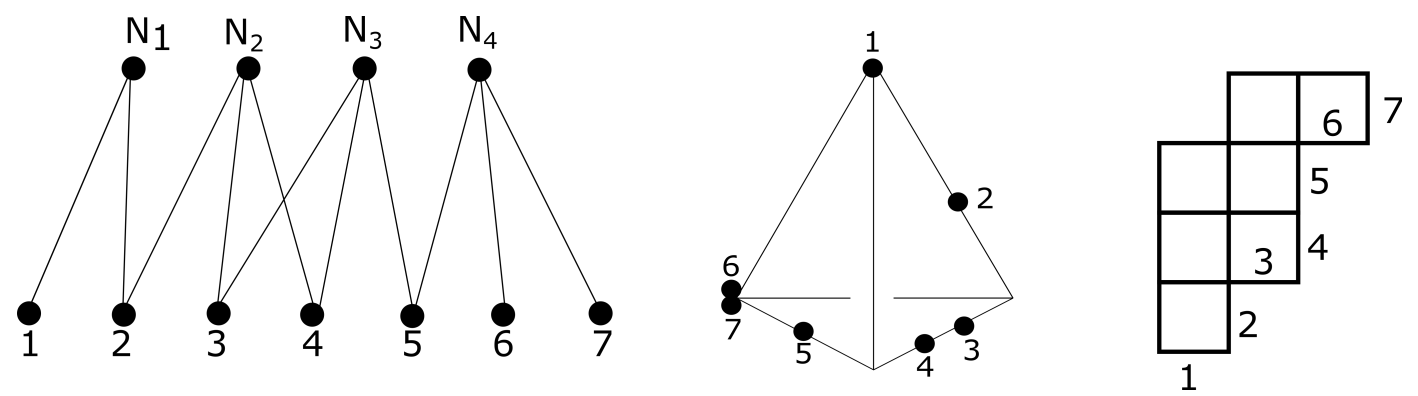

Figure 10.5: $M_{1}$ represented as a bipartite graph, on a simplex and as a lattice path presentation

(1) $\left(Z^{F_{1}}, \ldots, Z^{F_{4}}\right)=\left(N_{1}, N_{123}, N_{123}, N_{1234}\right)$ and $\left(Z^{B_{1}}, \ldots, Z^{B_{4}}\right)=$ 
$\left(N_{1234}, N_{1234}, N_{1234}, N_{4}\right)$.

(2) Now $\left(F_{1}, \ldots, F_{4}\right)=([1,2],[3,5],[3,5],[6,7])$ and $\left(B_{1}, \ldots, B_{4}\right)=$ $([1,4],[1,4],[1,4],[5,7])$.

(3) Then $X=\{1,3,4,6\}$.

(4) Hence, $\{1,3,4,6\}$ is a fundamental basis for $M$.

So a fundamental representation of $M_{1}$ on a simplex would be as illustrated in Figure 10.6 .

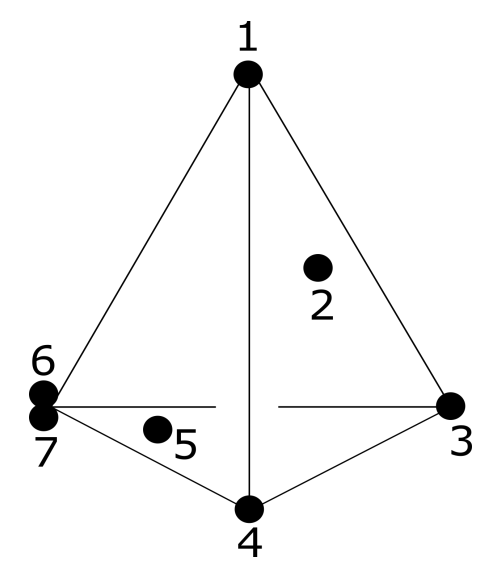

Figure 10.6: A fundamental representation of $M_{1}$

We now illustrate how the algorithm works for a lattice path matroid $M_{2}$ that is not fundamental (See Figure 10.7). Here, $E=[1,11]$ and $B=\{[1,3],[3,6],[5,7],[6,9],[8,11]\}$. For the sake of brevity, we again denote $N_{1}, \ldots, N_{i}$ as $N_{1 \ldots i}$.
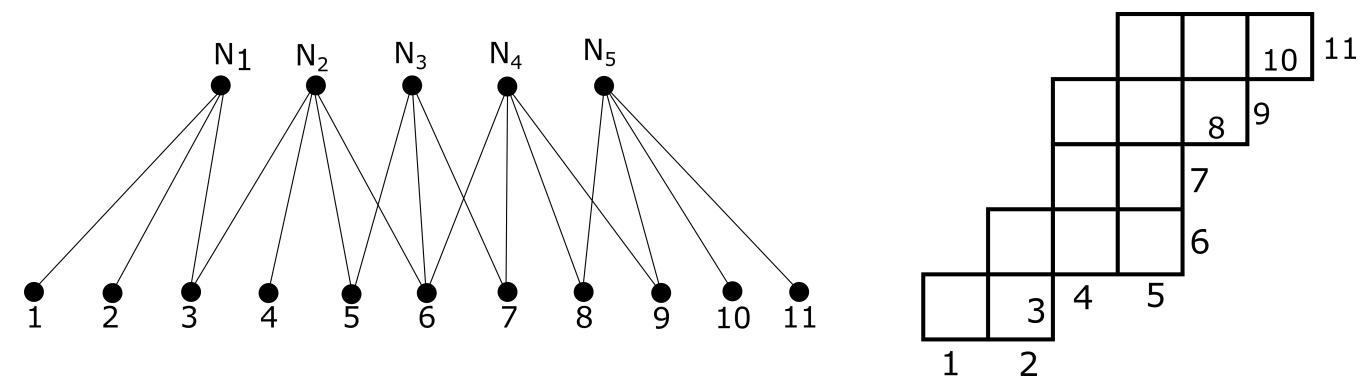

Figure 10.7: $M_{2}$ represented as a bipartite graph and as a lattice path presentation

The algorithm works as follows: 
(1) $\left(Z^{F_{1}}, \ldots, Z^{F_{5}}\right)=\left(N_{1}, N_{123}, N_{123}, N_{1234}, N_{12345}\right)$ and $\left(Z^{B_{1}}, \ldots, Z^{B_{5}}\right)=$ $\left(N_{12345}, N_{2345}, N_{345}, N_{345}, N_{5}\right)$.

(2) Now $\left(F_{1}, \ldots, F_{5}\right)=([1,3],[4,7],[4,7],[8,9],[10,11])$ and $\left(B_{1}, \ldots, B_{4}\right)=([1,2],[3,4],[5,7],[5,7],[8,11])$.

(3) Then the algorithm terminates without an output as the intersection $\left(N_{4} \cap F_{4} \cap B_{4}\right)$ is empty. Hence there exists no fundamental basis.

We observe that this matroid does not satisfy the condition in 9.1.3. Consider the two cyclic flats $F_{1}=[1,7]\left(=E-N\left(N_{5}\right)\right)$ and $F_{2}=[8,11](=$ $\left.E-N\left(N_{123}\right)\right)$. Then, $0=\mathrm{r}\left(F_{1} \cap F_{2}\right) \neq \mathrm{r}\left(F_{1}\right)+\mathrm{r}\left(F_{2}\right)-\mathrm{r}\left(F_{1} \cup F_{2}\right)=$ $4+2-5=1$. 


\section{Chapter 11}

\section{Fundamental bicircular matroids}

Even though bicircular matroids arise naturally from graphs, many properties have yet to be studied. We add to the literature by exploring the following natural question: what is the intersection of fundamental matroids and bicircular matroids? In other words, is there a polynomial-time algorithm to determine when a bicircular matroid is fundamental? This section answers this question positively.

We begin by proving a basic lemma about fundamental matroids.

Lemma 11.1.1. If $B$ is a fundamental basis in $M$ and $x$ is in $B$, then $B-x$ is a fundamental basis of $M / x$.

Proof. Let, if possible, $M / x$ not be fundamental. Then there exists an element $e$ that is in $E-B$ that is not freely placed in the flat spanned by the fundamental circuit $C_{M / x}\left(e, B^{\prime}\right)$ where $B^{\prime}=B-x$. Let $Z$ be a cyclic flat of $M / x$ such that $e$ is in $Z$ but $C_{M / x}\left(e, B^{\prime}\right) \nsubseteq Z$. Note that either $Z$ or $Z \cup x$ is a cyclic flat in $M$ that contains $e$. Also, the fundamental circuit of $e$ in $M$ is either $C_{M / x}\left(e, B^{\prime}\right)$, or it is $C_{M / x}\left(e, B^{\prime}\right) \cup x$. But this contradicts the fact that $M$ is fundamental as it implies that $e$ is not free in $C_{M}(e, B)$.

We now define two structures that are crucial in characterising bicircular matroids that are fundamental. Note that when $X$ is a set of edges, $G[X]$ is the subgraph induced by $X$, whose edge set is $X$ and vertex set is the set of vertices that are endpoints of edges in $X$. 
Definition 11.1.2. Let $G$ be a graph. A spider is a set of edges $X$, such that $G[X]$ contains a single loop and no other cycles. Furthermore, if $v$ is the vertex incident with the loop, every vertex in $G[X]$ other than $v$ is joined to $v$ by a single edge in $X$. Also, if $u$ is in $G[X]$ and is not $v$, there is at most one edge incident with $u$ but not $v$.

Note that if $G$ is a graph with minimum degree at least three, then the vertices adjacent to the loop-vertex in the spider are joined to the loop-vertex by at least two edges each.

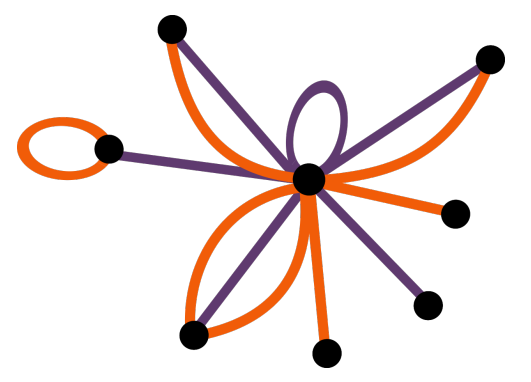

Figure 11.1: An example of a spider $X$. The purple edges are in $X$ while the orange edges are in $G$ but not $X$.

Definition 11.1.3. A hammock consists of two edges joining distinct vertices $u$ and $v$, where there is at most one edge incident with $u$ but not $v$, and at most one edge incident with $v$ but not $u$.

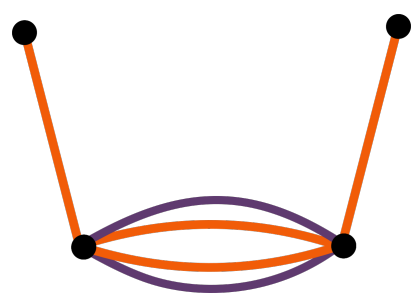

Figure 11.2: An example of a hammock $X$. The purple edges are in $X$ while the orange edges are in $G$ but not $X$.

We blur the distinction between a set of edges $X$ and $G[X]$ when doing so will not create confusion.

Definition 11.1.4. A good component of a graph $G$ is either a spider or a hammock. A good basis $B$ of $B(G)$ is one in which every component of $G[B]$ is good. 
We now prove an inductive connectivity tool that will prove useful in the main theorem. This lemma can be derived as a corollary from [33]. However we provide an independent proof here.

Theorem 11.1.5. Let $M$ be a 3-connected matroid up to parallel pairs and loops. Let $B$ be a basis. There exists $x \in B$ such that $M / x$ is 3-connected up to parallel pairs and loops.

Proof. Let $x$ be an arbitrary element in $B$. Then we assume that $M / x$ has a 2-separation $(U, V)$ with $r(U), r(V)>1$, else there is nothing to prove. Clearly, $(U \cap x, V)$ and $(U, V \cup x)$ are 3-separations of $M$ with $r(U), r(V)>$ 2 and $x \in \mathrm{cl}(U) \cap \mathrm{cl}(V)$. Neither $U$ nor $V$ is spanning and we have chosen $x$ so that $|U|$ is as small as possible. Consider $y \in(U \cap B)-c l(V)$. Such an element exists as $V$ is not spanning. Then there exist 3-separations $\left(U^{\prime}, V^{\prime} \cup y\right)$ and $\left(U^{\prime} \cup y, V^{\prime}\right)$ of $M$ with $y \in c l\left(U^{\prime}\right) \cap c l\left(V^{\prime}\right)$. Assume without loss of generality that $x \in U^{\prime}$.

Case 1: $\left|V \cap V^{\prime}\right| \geq 2$.

This implies that $V \cup V^{\prime}$ is a 3-separation by Lemma 2.1 [30]. Thus, the complement $\left(U \cap U^{\prime}\right) \cup\{x, y\}$ is also a 3-separation. We also have $\mid V \cap$ $\left(V^{\prime} \cup y\right) \mid \geq 2$. Then, by the same reasoning as above, $V \cup V^{\prime} \cup y$ and $\left(U \cap U^{\prime}\right) \cup x$ are 3 -separating. In a similar fashion, we can prove that $(U \cap$ $\left.U^{\prime}\right) \cup y$ and $U \cap U^{\prime}$ are 3-separating sets.

By Lemma 2.3 [30], $y \in c l\left(U \cap U^{\prime}\right)$ or $y \in c l^{*}\left(U \cap U^{\prime}\right)$. Also, since $y \in$ $c l\left(V^{\prime}\right), y \in E-\left(\left(U \cap U^{\prime}\right) \cup y\right)$. But this implies $y \notin c l^{*}\left(U \cap U^{\prime}\right)$ by Lemma 2.2 [30], which in turn implies that $y \in \operatorname{cl}\left(U \cap U^{\prime}\right)$.

Now, $\left(U \cap U^{\prime}, V \cup V^{\prime} \cup y\right)$ and $\left(\left(U \cap U^{\prime}\right) \cup y, V \cup V^{\prime}\right)$ are 3-separations in $M$, where $y$ is in $c l\left(U \cap U^{\prime}\right) \cap \operatorname{cl}\left(V \cap V^{\prime}\right)$. Our choice of $|U|$ implies $U \cap U^{\prime}=U$ which in turn implies that $U \subseteq U^{\prime}$ and thus $V^{\prime} \subseteq V$. But $y \in \operatorname{cl}\left(V^{\prime}\right)$ and $y \in \operatorname{cl}(V)$, which is a contradiction.

Case 2: $\left|V \cap V^{\prime}\right| \leq 1$

Then, $\left|U^{\prime} \cap V\right| \geq 2$ (as $r(V) \geq 2$ ). Hence, $U \cup V^{\prime}$ and $\left(U \cup V^{\prime}\right) \cup y$ are 3-separations by similar arguments as the previous case. Hence, by Lemma 2.3 [30] again, $y \in c l\left(U \cap v^{\prime}\right)$ or $y \in c l^{*}\left(U \cap V^{\prime}\right)$. Since, $y \in c l\left(U^{\prime}\right)$, $y \in E-\left(\left(U \cap V^{\prime}\right) \cup y\right)$. Thus $y \notin c l^{*}\left(U \cap V^{\prime}\right)$ and this implies that $y \in \operatorname{cl}\left(U \cap V^{\prime}\right)$. 
Again, by our choice of $U$, we obtain that $U=U \cap V^{\prime}$ which implies that $U^{\prime}$ is a subset of $V$. Then, $\operatorname{cl}\left(U^{\prime}\right) \subseteq \operatorname{cl}(V)$. But this leads to the false conclusion that $y \in c l(V)$ as $y \in \operatorname{cl}\left(U^{\prime}\right)$.

We are in good shape to characterise fundamental bases in bicircular matroids. We begin with 3-connected matroids. Note that the term digon is used to refer to two element cycles.

Theorem 11.1.6. Let $G$ be a graph with no cut vertex, and minimum degree at least three. Then $B$ is a fundamental basis of $B(G)$ if and only if $B$ is good.

Proof. Let $B$ be a basis of the bicircular matroid $B(G)$. Note that a component of $G[B]$ is bad if it is not good. Let $X$ be a bad component of $G[B]$. Then $X$ must satisfy one of the following (In the following figures, red edges are in $X$ and green edges are in $G-B$ ):

- X contains a cycle with more than two edges.

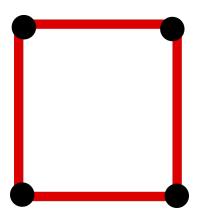

- $X$ contains a cycle of two edges, and $X$ contains more than two edges.

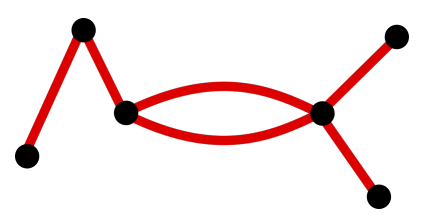

- $X$ consists of a cycle of two edges, joining $u$ to $v$, where $u$ is incident with two edges that are not incident with $v$.

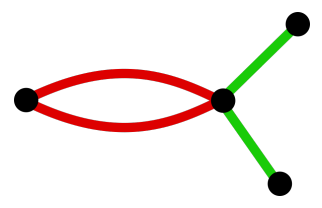

- $X$ contains a loop incident with vertex $v$ and $u$ is a vertex in $G[X]$ that is not joined to $v$ by an edge in $X$. 


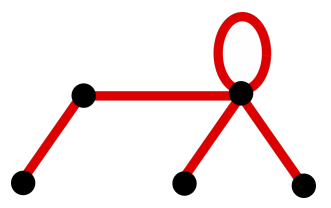

- $X$ contains a loop incident with $v$, and every vertex in $G[X]$ is adjacent to $v$ in $G[X]$, but there exists a vertex $u$ in $G[X]$ such that $u$ is incident with two edges that are not incident with $v$.

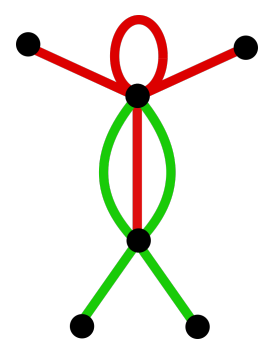

We first prove that contracting an element outside a bad component does not affect the 'badness' of that component after contraction.

Claim 11.1.6.1. Let $G$ be a graph with no cut vertex and minimum degree at least three. If $X$ is a bad component of a basis $B$ of $B(G)$, and $x$ is in $B-X$, then $X$ is a bad component of $B-x$ in $G / x$.

Proof. Let $x$ be in a component $X^{\prime}$ of $B$. We examine each of the different types of bad components mentioned above and verify that $X$ is a bad component of $G / x$ :

(i) Let $X$ contain a cycle with more than two edges. Then $B-x$ still contains the same cycle $X$ in $G / x$.

(ii) Let $X$ contain a cycle with two edges with a total of more than two edges. Then contracting $x$ keeps the cycle intact and does not affect any of the edges that are in $X$ but not in the cycle. Hence, $B-x$ still has a bad component in $G / x$.

(iii) Let $X$ consist of a cycle of two edges, joining $u$ to $v$, where $u$ is incident with two edges that are not incident with $v$. Again contracting $x$ does not affect the parallel pair and the multiple edges incident with one of the end vertices. 
(iv) $X$ contains a loop at vertex $v$ and there exists a vertex $u$ in $X$ not adjacent to $v$ in $X$. Then, contracting $x$ will not affect the loop nor the path of length two in $X$ and hence $X$ remains the same bad component.

(v) $X$ contains a loop incident with $v$, and every vertex in $G[X]$ is adjacent to $v$ in $G[X]$, but there exists a vertex $u$ in $G[X]$ such that $u$ is incident with two edges that are not incident with $v$. In this case, the contraction of $x$ may affect one or more of the edges adjacent to $u$, converting them into loops. But this still does not change the fact that $u$ is adjacent with more than one edge that are not adjacent to $v$.

Now, let $G$ be a minimal counterexample to Theorem 11.1 .6 and $B$ be a fundamental basis of $G$. Clearly, $G[B]$ must contain a bad component, say $H$.

If $e$ is an edge not in $B$, then $G \backslash e$ has either a cut-vertex, or a vertex with degree two, due to the minimality of $G$. This holds true because $B$ is a fundamental basis in $B(G) \backslash e$ and $B$ still has a bad component in $G \backslash e$.

The following observation will greatly simplify the structure of parallel classes in G:

Claim 11.1.6.2. All parallel classes of $G$ have size at most two.

Proof. Assume that $G$ has a parallel class of size at least three, with the end vertices labelled $u$ and $v$. Clearly at most two edges of the parallel class can be part of $B$. Let $x$ be an edge joining $u$ and $v$ that is not in $B$. If there exists a degree two vertex in $G \backslash x$, it has to be either $u$ or $v$. Assume without loss of generality that it is $u$. In $G, u$ is only incident with the two edges in $B$ joining $u$ to $v$ and $x$ as $u$ has degree two in $G \backslash x$. This implies that $v$ is a cut-vertex in $G \backslash x$ which in turn implies that $v$ is a cut-vertex in $G$, a contradiction. Hence, deleting an edge in this parallel class does not create a graph with a vertex with degree two.

We further observe that deleting $x$ cannot create a graph with a cut-vertex either as follows: Suppose that $G \backslash x$ has a cut-vertex, say $c$. Then, we can find vertices $a$ and $b$ such that every path from $a$ to $b$ passes through $c$. 
Let $P$ be a path from $a$ to $b$ in $G$ that does not use $c$. Such a path exists as $G$ has no cut vertices. If this path does not contain $x$, then $P$ is a path that avoids $c$ in $G \backslash x$ too, a contradiction. Since $x$ is in a parallel pair, if $P$ contains $x$, then $G \backslash x$ contains a path that connects $a$ and $b$ and avoids $c$, a contradiction.

Claim 11.1.6.3. Let $x$ be an edge in $B$ such that $G / x$ has no cut-vertex, and minimum degree at least three. Then any bad component of $G[B]$ must contain $x$.

Proof. Let $X$ be a bad component of $B$ such that $x$ is not in $X$. Then, by Lemma 11.1.6.1, $X$ is still a bad component of $B-x$ in $G / x$. But, $G / x$ has no cut-vertex and has minimum degree three. This contradicts the minimality of $G$. Hence, $x$ is an edge in the bad component $X$.

The above claim implies that there is exactly one bad component of $G[B]$. This component, say $H$, contains $x$, which is an edge that can be contracted to obtain a minor with no cut-vertex and has minimum degree at least three by Theorem 11.1.5.

Claim 11.1.6.4. Every component of $G[B]$ other than $H$ is a single loop.

Proof. Assume otherwise. Let $H^{\prime}$ be a hammock in $B$. Assume that contracting an edge in $H^{\prime}$, say $y$, produces a graph with a cut-vertex, say $c$. Then, there exists vertices $a$ and $b$ in $G / y$ such that every path from $a$ to $b$ passes via $c$. But if $C$ is a cycle that contains $a$ and $b$, the existence of which is guaranteed by the fact that $G$ has no cut-vertices, then $y$ is a chord in $C$. This implies that $u$ and $v$ have at least three neighbours in $G$, which is a contradiction. Thus, contracting any edge in $H^{\prime}$ leaves a graph with no cut-vertex, and minimum degree at least three which is another contradiction. This is illustrated in Figure 11.3 . 

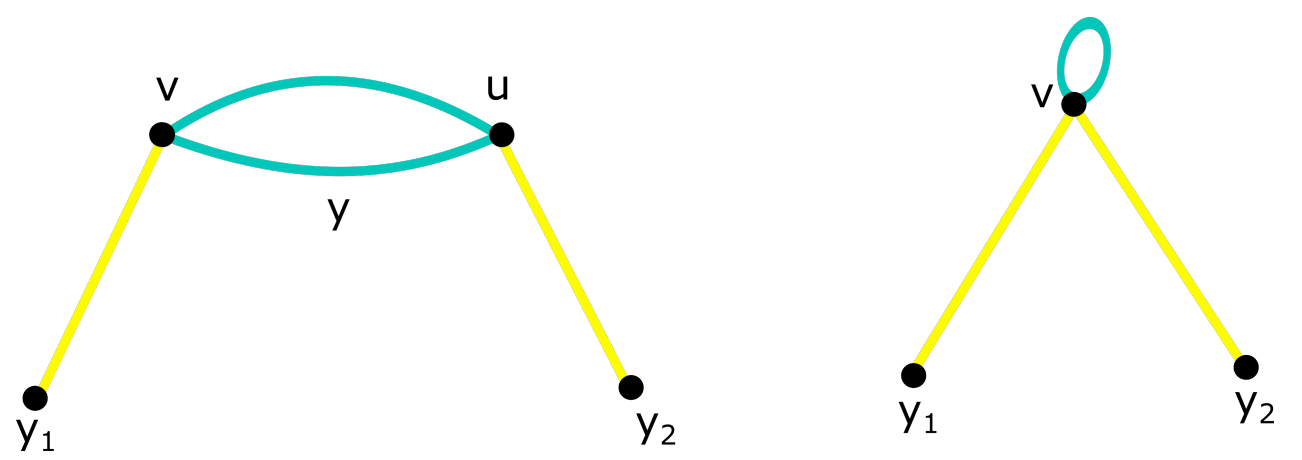

Figure 11.3: $H^{\prime}$ on the left and the resulting component in $G / x$. All of $v$, $y_{1}$ and $y_{2}$ have degree at least 3 in $G$ and its minor.

This implies that any other component is a spider. Assume that a spider contains a non-loop edge, $x^{\prime}$. Let $v$ be the loop vertex and $u$ be the other end-vertex of $x^{\prime}$. Let, if possible, $c$ be a cut vertex of $G / x^{\prime}$. Then there exist vertices $a$ and $b$ such that every path through $a$ and $b$ passes thorugh $c$. And by similar argument as above, $x^{\prime}$ is a chord in the cycle that contains $a$ and $b$ in $G$. This is a contradiction as $u$ is joined to at most one vertex that is not $v$. Thus, $G / x^{\prime}$ has no cut vertex, and minimum-degree at least three, which is another contradiction. Hence the claim holds.

We now analyse each of the different types of bad components.

Case $1 . H$ contains a cycle with more than two edges.

Since $G / x$ has no bad component, $H$ must be a triangle. $H / x$ is a good parallel pair in $G / x$, so there are at most two edges joining vertices in $H$ to vertices not in $H$. We refer to Figure 11.4 for edge labels. Note that $x$ cannot join $u$ to $v$, or else, $G / x$ has a cut-vertex. 


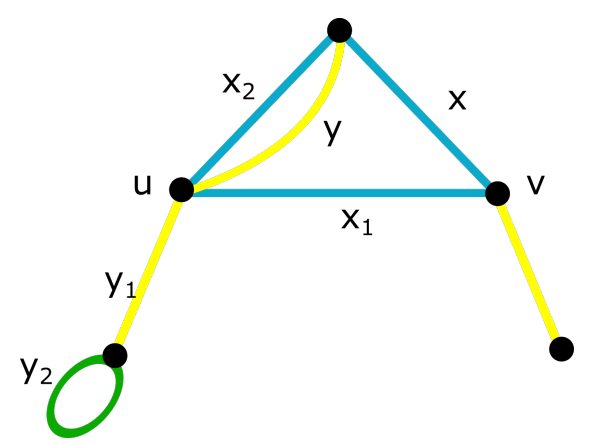

Figure 11.4: Blue edges are in $H$, green edges in $B-H$ and the yellow edges are not in $B$

There has to exist an edge $y$ as there are no degree two vertices in G. Also, there is no loop incident with $u$ or $v$, by the definition of a hammock. Now, the fundamental circuit of $y$ with respect to $B$ is the theta graph consisting of edges $x, y, x_{1}, x_{2}$. Then the closure of the loose handcuff that contains the edges $y, y_{1}, y_{2}$ does not span the fundamental circuit of $y$ which is a contradiction to the fact that $B$ is a fundamental basis.

Case 2. $H$ contains more than two edges along with a cycle of two edges. Let $u$ and $v$ be the end vertices of the two-cycle. Then there is at least one more edge in $H$ incident to one of $u$ or $v$, say $y$. Then $H / y$ forms a good component in $G / y$ if and only if $H / y$ is a hammock. Say $y$ is incident with $w$. Then $H$ has three edges and $w$ has degree at most two in $G$ (by definition of hammock), a contradiction. Hence, $H / y$ does not form a good component in $G / y$. So we choose one of the edges in the two-cycle, say $x$. Then $H / x$ is a good component in $G / x$. We refer to Figure 11.5 for labels. 


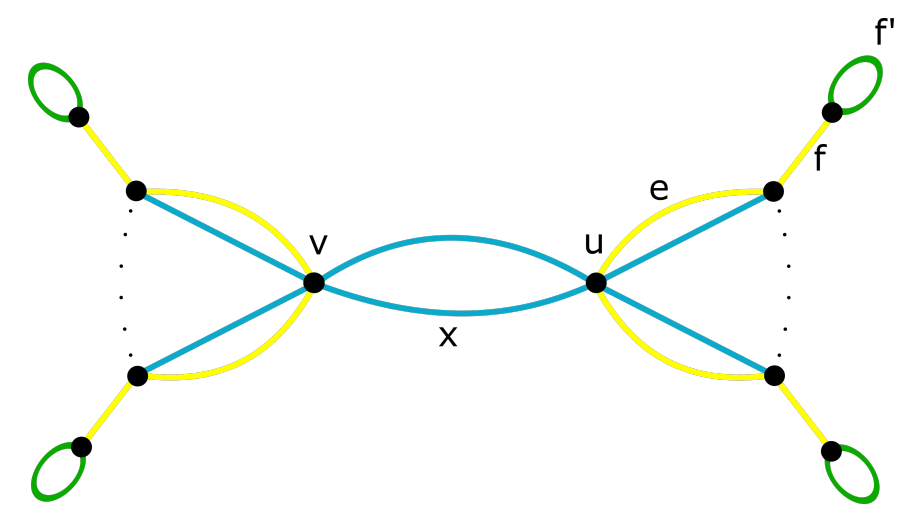

Figure 11.5: Blue edges are in $H$, green edges in $B-H$ and the yellow edges are not in $B$

Consider $e$ that is in parallel with an edge from $H$. Such an edge exists, else $H / x$ will not be a spider. Then the fundamental circuit of $e$ is the tight handcuff that consists of $x$, the edge in $H$ parallel to $x, e$ and the edge in $H$ parallel to $e$. Consider the loose handcuff that consists of $e$, the edge in $H$ parallel to $e, f$ and $f^{\prime}$. This circuit shows that $e$ is not free in its fundamental circuit which is a contradiction.

Case 3. $H$ consists of a cycle of two edges, joining $u$ to $v$, where $u$ is incident with at least two edges that are not incident with $v$.

Clearly $x$ is an edge in the parallel pair and $H-x$ is a spider in $G / x$. We refer to Figure 11.6 for labels.

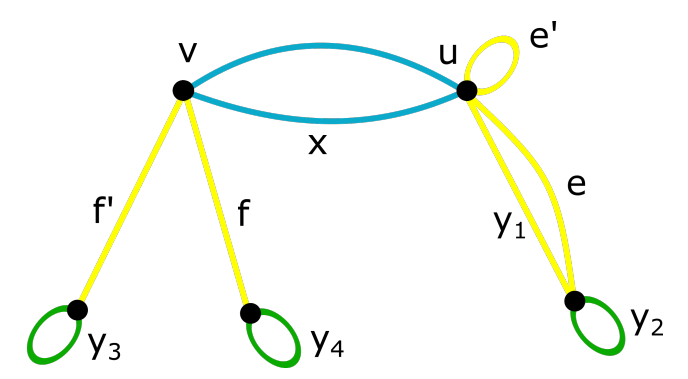

Figure 11.6: Blue edges are in $H$, green edges in $B-H$ and the yellow edges are not in $B$

We have three possible situations in this case: (i) either one end vertex, $u$ in the figure, is joined to a vertex that is not $v$ by a parallel pair or (ii) an end vertex of the parallel pair, $v$ in the figure, is joined to at least two distinct 
vertices or (iii) an end vertex, $u$ in the figure, is incident with a loop and another edge.

Consider the first situation. Here $e$ is an edge in the parallel pair incident with $u$ and the fundamental circuit of $e$ consists of the edges $x$, the edge in parallel with $x, e$ and $y_{2}$. But the tight handcuff formed by $e, y_{1}$ and $y_{2}$ is evidence that $e$ is not free in this fundamental basis.

In the second scenario, consider $f$, an edge incident with $v$. Then the fundamental circuit of $f$ consists of $x$, the edge parallel to $x, f$ and $y_{4}$. Now consider the loose handcuff that consists of $y_{3}, f^{\prime}, f$ and $y_{4}$. This is a circuit that contains $f$ whose closure does not span the fundamental circuit of $f$ which is a contradiction.

In the final case, let $e^{\prime}$ be the loop incident with $u$. Then $u$ has to be incident with a second edge for $H$ to be bad. If this edge is a loop, then $u$ is incident with two loops outside $B$ and this makes $v$ a cut-vertex in $G$, a contradiction. Hence, $u$ is incident with $e^{\prime}$ and non-loop edge, say $e$. Then the fundamental circuit $C\left(e^{\prime}, B\right)$ is the tight handcuff that consists of $x$, its parallel edge and $e^{\prime}$. But this circuit is not in the closure of the span of $\left\{e, e^{\prime}, y_{1}\right\}$, which is a circuit containing $e^{\prime}$, a contradiction.

Case 4. $H$ contains a loop and a vertex that is not joined to the loop-vertex by an edge in the component

In this case, we will argue in the following paragraphs, that avoiding degree two vertices and making sure that there is an edge $x$ such that $H-x$ is a good component of $G / x$ gives rise to a set of edges as in Figure 11.7 .

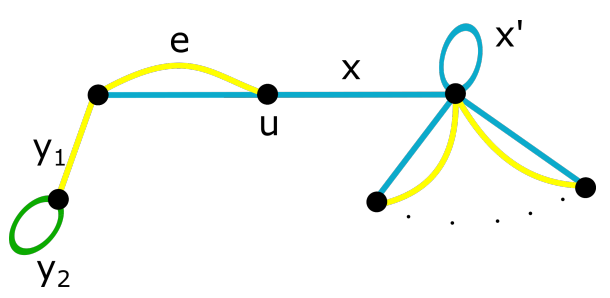

Figure 11.7: Blue edges are in $H$, green edges in $B-H$ and the yellow edges are not in $B$

The existence of $e$ is justified by the minimum degree condition imposed on $G$. We aim to obtain a spider after contracting $x$, which implies that 
we cannot have a path of length two beginning at the loop-vertex in $H / x$. Note that $x$ cannot be the edge parallel to $e$ as contracting $x$ would imply that $u$ is a cut-vertex in $G / x$. Thus $x$ is the edge incident with the loop-vertex and the edge in $H$ that it is not incident with the loop-vertex. We also argue that the edge $x^{\prime}$ is not an apt choice for $x$ as we prove that if $G^{\prime}$, the graph obtained by contracting $x^{\prime}$, has no cut-vertices and has at least minimum degree 3 , then so does $G / x$. As explained in Chapter 2. contracting the loop of a graph corresponding to a bicircular matroid is different to edge contraction in graphs. Essentially, $x^{\prime}$ is deleted and every edge adjacent with $x^{\prime}$ is now a loop at the other end vertex. Assume that $G^{\prime}$ has no cut-vertices. Then there exists no vertex $v$ such that every path through a pair of vertices passes through $v$. Thus, if $G / x$ has a cut-vertex, it has to be the loop vertex. This implies that $G$ has a cut-vertex, which is a contradiction. Now assume that $G^{\prime}$ has no vertex of degree less than three. Clearly, if this is the case, then the same is true for $G / x$ and hence the claim holds true.

Now, considering the edge $e$, we observe that the fundamental circuit is the loose handcuff that consists of $e$, the edge in $H$ parallel with $e, x$ and $x^{\prime}$. But the loose handcuff $y_{2}, y_{1}, e$ and the edge in $H$ parallel with $e$ contradict the fact that $e$ is free in $B$.

Case 5. $H$ contains a loop, and every vertex in the component is joined to the loop-vertex by an edge of the component and there exists a vertex $u$ in the component such that $u$ is incident with two edges that are not incident with the loop-vertex.

In this case, we have a vertex $u$ that can be incident with a combination of a loop, a parallel pair and a single edge such that $u$ is incident with at least two vertices that are not the loop-vertex. This is pictured in Figure 11.8 . We use the labels in the said figure to complete the argument. 


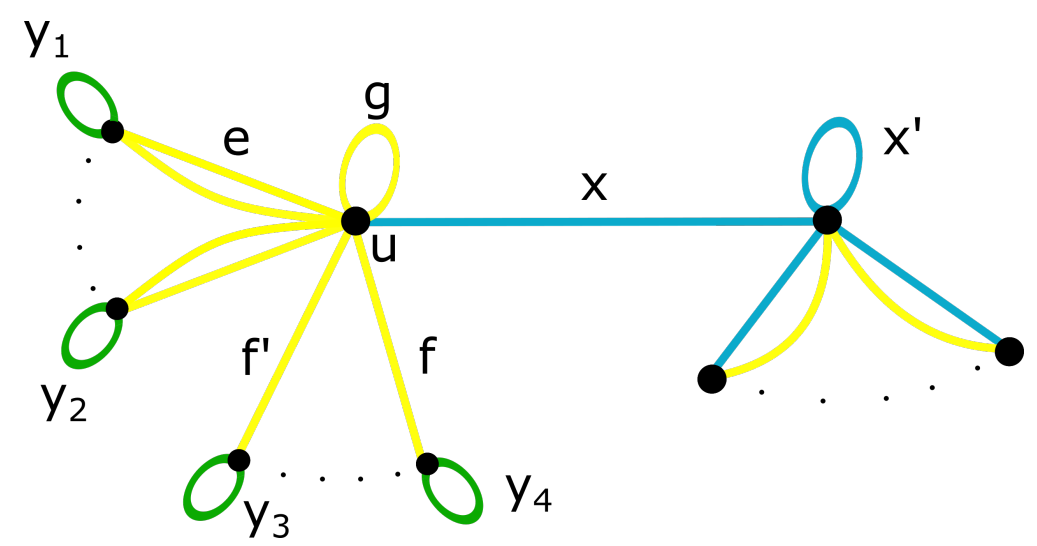

Figure 11.8: Blue edges are in $H$, green edges in $B-H$ and the yellow edges are not in $B$

Again, $x^{\prime}$ is not chosen to be contracted because $x$ is a better choice as argued previously.

Consider an edge $e$ in a parallel pair incident with $u$. Then the fundamental circuit $C(e, B)$ consists of $y_{1}, e, x$ and $x^{\prime}$. But the closure of the tight handcuff that consists of the parallel pair that contains $e$ and $y_{1}$ does not contain $C(e, B)$, this is a contradiction.

Now consider an edge $f$ that is a single edge incident with $u$. Then there is either a loop, say $g$, or a non-loop edge, say $f^{\prime}$ incident with $u$. Then, the fundamental circuit $C(f, B)$ consists of $y_{4}, f, x$ and $x^{\prime}$. But, neither of the closures of the loose handcuffs $g, f, y_{4}$ or $y_{3}, f^{\prime}, f, y_{4}$ spans $C(f, B)$ which leads to another contradiction.

Finally, consider a loop $g$ incident with $u$. Then there is another edge, say $e$ incident with $u$. The fundamental circuit of $g$ is the loose handcuff $g, x, x^{\prime}$. If $e$ is a loop, then the closure of the tight handcuff $e, g$ does not contain $C(g, H)$, which is a contradiction. If $e$ is a non-loop edge, then the loose handcuff $y_{1}, e, g$ does not span $C(g, H)$ which is another contradiction and rules out this case.

With this we come to the conclusion of the case analysis. We have arrived at a contradiction for each of the possible bad components.

To prove the converse, that is, a good basis is fundamental, we describe the fundamental circuits relative to a good basis, and observe that every element is free in its fundamental circuit. Any edge whose fundamental 
circuit is a loose handcuff with loops at either end, will be free in this circuit as any cyclic flat will contain the span of this fundamental circuit. Else, if an edge has a fundamental circuit that is a loose handcuff consisting of digons at the end, then every cyclic flat contains the closure of this loose handcuff. Otherwise, if an edge has a fundamental circuit that is a loose handcuff with a spider at one end and a digon at the other, then this element is again free in the span of its fundamental circuit.

Clearly, if an edge $e$ is in a parallel class that contributes to a hammock in the basis, then any circuit closure that contains $e$ will also contain the theta graph that consists of the hammock and edge $e$, which is the fundamental circuit of $e$. Lastly, if $e$ has a tight handcuff as its fundamental circuit, then it is a non-loop edge that is in a parallel class with an edge in the spider. Since the parallel class is incident with exactly one other edge that is not incident with the loop vertex, any cyclic flat that contains this edge, contains the fundamental circuit too.

We have now characterised 3-connected bicircular fundamental matroids. Before moving on and characterising fundamental bicircular matroids, we devise a simple algorithm that will construct a fundamental basis in a bicircular matroid if the same exists or otherwise terminate with no output. Let $G$ be a graph and $B(G)$ be its bicircular matroid. Then the algorithm ss the following:

\section{Algorithm: FUNDAMENTAL 3-CONNECTED BICIRCULAR MATROIDS}

Input: The graph $G$

Output: A fundamental basis, $X$ of $B(G)$

Step I Initialise $X$ to be empty. Scan every vertex of the graph and if any vertex has a single loop adjacent to it, this loop is added to the set $X$.

Step II Scan the vertices that are not incident with any edge in $X$. If it has exactly two neighbours: a vertex joined by a single edge and another vertex by a parallel pair whose other end vertex is incident with exactly a single edge, then the parallel pair is added to $X$. Otherwise, if it has degree 2 by being adjacent to a vertex by a single edge and 
another vertex by a parallel class whose other end vertex is incident with a loop in $X$, then an edge of the parallel class is added to $X$.

Step III If there is a vertex that is still not incident with an edge in $X$, then $B(G)$ is not a fundamental matroid. Else, $X$ is a fundamental basis of $B(G)$.

If there is a basis consisting of spiders and hammocks, this algorithm will find it. Otherwise, no such basis exists. This polynomial time algorithm works in about $O(n)$ time (where $n$ is the number of vertices in $G$ ) and we now aim to achieve the same for matroids that are not 3-connected. We first consider graphs that have no cut-vertices, and hence are 2-connected, but have a degree-2 vertex. Clearly, such vertices are incident with edges in a series pair. We now prove results about fundamental bases in matroids with series pair. We essentially prove that having a series pair does not affect the fundamental nature of a matroid.

Lemma 11.1.7. Let $\{x, y\}$ be a series pair in a matroid $M$. Then $M$ is fundamental if and only if $M / x$ is fundamental.

Proof. Let $M$ be a fundamental transversal matroid and $B$ be a fundamental basis. Then, as $\{x, y\}$ is a cocircuit and intersects every basis, $B \cap\{x, y\}$ is non-empty. Since $M / x$ and $M / y$ are isomorphic, then without loss of generality let $x \in B \cap\{x, y\}$. Then, by Lemma 11.1.1, $M / x$ is fundamental.

Now assume that $M / x$ is fundamental and let $B$ be a fundamental basis. Then every element $e$ in $E-(B \cup x)$ is freely placed in the span of $C_{M / x}(e, B)$. We want to show that it is freely placed in the span of $C_{M}(e, B \cup x)$. Assume otherwise, that is, $e$ is not free in the span of its fundamental circuit relative to $B \cup x$. Note that if $C$ is a circuit in $M / x$, then either $C$ or $C \cup x$ is a circuit in $M$. Hence, if $Z$ is a cyclic flat in $M / x$, then either $Z$ or $Z \cup x$ is a cyclic flat in $M$. Conversely, if $C$ is a circuit of $M$ containing $x$, then $C-x$ is a circuit in $M / x$. If $C$ does not contain $x$, then $C$ is a union of circuits in $M / x$. Let $Z$ be a cyclic flat in $M$. If $x$ is in $Z$, then $Z-x$ is a cyclic flat of $M / x$. If $x$ is not in $Z$, then $Z$ is a cyclic flat in $M / x$. Let $C=C_{M / x}(e, B)$ be the fundamental circuit of $e$ in $M / x$. Hence, in $M$, its fundamental circuit, $C^{\prime}$, is either $C$ or $C \cup x$. Let $Z$ be a cyclic flat $M$ 
such that $e$ is in $Z$, but $C^{\prime}$ is not contained in $Z$. Then, $Z$ or $Z-x$ is a cyclic flat in $M / x$ that contains $e$ but does not contain $C$, which is a contradiction to the fact that $B$ is a fundamental basis of $M / x$.

The following corollary follows easily from the above lemma:

Corollary 11.1.8. Let $G$ be a graph and $G^{\prime}$ be the graph obtained by contracting all but one edge from each series class in $B(G)$. Then, $B(G)$ is fundamental if and only if $B\left(G^{\prime}\right)$ is fundamental.

Thus, to find a fundamental basis in a 2-connected graph with vertices of degree 2, we contract the series pairs until we are left with a graph that has minimum degree 3 . Then, we can apply the previously mentioned algorithm to find a fundamental basis if it exists.

Now we move on to finding a fundamental basis in graphs that have cut vertices. We do so by considering the block-cut-vertex tree of the corresponding graph.

Definition 11.1.9. Let $G$ be a graph. A block of $G$ is a maximal 2-connected subgraph. A block-cut-vertex tree of $\mathrm{G}, \mathrm{bc}(\mathrm{G})$ is a tree where every vertex is either a cut-vertex or a block of $G$ and a block vertex is adjacent to a cut-vertex if and only if the cut-vertex is in the block.

Now we prove a lemma that will help simplify the proof of characterisation of fundamental bicircular matroids. This

Lemma 11.1.10. Let $M$ be a connected matroid with a fundamental basis $B$. Also, let $M=M_{1} \oplus_{2} M_{2}$ along the base-point $e$. Then, up to relabelling, $e$ is in a parallel pair with $e^{\prime}$ in $M_{1}, e^{\prime} \in B$ and $B \cap E\left(M_{1}\right)$ is a fundamental basis of $M_{1}$ and $\left(B \cap\left(M_{2}\right)\right) \cup e$ is a fundamental basis of $M_{2}$.

Proof. Without loss of generality, let $B \cap E\left(M_{1}\right)$ span $E\left(M_{1}\right)-e$ in $M$. Since $r_{M}\left(\left(M_{1} \cup M_{2}\right) \backslash e\right)=r_{M_{1}}\left(M_{1}\right)+r_{M_{2}}\left(M_{2}\right)-1$, this implies that $r_{M}\left(B \cap E\left(M_{1}\right)\right)=r_{M_{1}}\left(M_{1}\right)$ and hence $r_{M}\left(B \cap E\left(M_{2}\right)\right)=r_{M_{2}}\left(M_{2}\right)-1$. Then $B \cap M_{2}$ spans a hyperplane of $M_{2}$. But $\mathrm{cl}_{M}\left(E\left(M_{2}\right)-e\right)$ is a cyclic flat of $M$. But $B \cap E\left(M_{2}\right)$ does not span this flat. Hence $\operatorname{cl}_{M}\left(E\left(M_{2}\right)-e\right)$ contains an element of $B \cap E\left(M_{1}\right)$, say $e^{\prime}$. Then $e^{\prime}$ is parallel to $e$ in $M_{1}$. Now every element in $E(M)-B$ is free in its fundamental circuit 
relative to $B$. Hence, every element in $E\left(M_{2}\right)-B$ is free with respect to its fundamental circuit in $M_{2}$ relative to the basis $\left(B \cap E\left(M_{2}\right)\right) \cup$ e. Similarly, every element in $E\left(M_{1}\right)-B$ is free with respect to its fundamental circuit in $M_{1}$ relative to the basis $\left(B \cap E\left(M_{1}\right)\right)$.

We can now prove the main result in this section: finding a fundamental basis in a bicircular matroid.

Theorem 11.1.11. Let $G$ be a graph and $B$ a basis of $B(G)$. Then $B$ is a fundamental basis of $B(G)$ if and only if $B \cap E(H)$ is a fundamental basis of $H$ for each block $H$ and $B$ contains a loop incident with each cut-vertex incident with $H$.

Proof. Let $B$ be a basis of $B(G)$. We begin by assuming that each block $H$ of $G$ has a fundamental basis $B_{H}=B \cap E(H)$ with a loop at every vertex. We want to show that the union of all $B_{H}, B$, is indeed a fundamental basis of $B(G)$. Consider the block-cut-vertex tree of $G$. Consider an element $e$ in $H$. Then, as $B_{H}$ is a fundamental basis of $H, e$ is free in $C_{H}\left(e, B_{H}\right)$, the fundamental circuit of $e$ with respect to $B_{H}$. Thus, any cyclic flat in $H$ that contains $e$, contains $C\left(e, B_{H}\right)$. Since $B \cap H$ spans $B(H)$, the fundamental circuit $C(e, B)$ is equal to $C_{H}\left(e, B_{H}\right)$. Also, if $Z$ is a cyclic flat of $M$, then $Z \mid H$ is a cyclic flat of $B(H)$ (this relies on having a loop at every cut-vertex). Now, $B_{H}=B \cap H$ and hence, $C_{B}(e, B)=C_{H}\left(e, B_{H}\right)$. Since $e$ is free in $C_{H}\left(e, B_{H}\right)$, e is now free in $C_{B}(e, B)$ too. This proves that $B$ is a fundamental basis.

Now assume that $B$ is a fundamental basis of $B(G)$. We aim to prove that $B \cap E(H)$ is a fundamental basis for each block $E(H)$ and $B$ contains a loop incident with each cut-vertex incident with $H$. We prove this by induction on the number of vertices, $n$, in the block-cut-vertex tree of $G$. We begin with $G$ that has a single block. Then by Theorem 11.1.6, $G$ has a fundamental basis that consists of spiders and hammocks.

We proceed by assuming that the result holds true for $n$ and we aim to prove this for $n+1$. Being a tree, we can always find a leaf in $b c(G)$ that corresponds to a block $H$ in $G$ such that it is joined to the rest of $G$ by a single cut-vertex. Then $H$ and $H^{\prime}$, where $H^{\prime}$ is the union of every block in $G$ that is not $H$, form two subgraphs of $G$, both of which have less than 
$n+1$ cut-vertices. Then, two blocks of $G$ joined together by the cut-vertex is a 2-sum in the corresponding matroid $B(G)$. Thus by the induction hypothesis and Lemma 11.1.10, $B \cap H$ and $B \cap H^{\prime}$ are fundamental bases for $H$ and $H^{\prime}$ respectively with a loop at the cut-vertex that joins $H$ and $H^{\prime}$. Hence proved.

Corollary 11.1.12. Let $B(G)$ be a bicircular matroid on graph $G$. Then, $B(G)$ is a fundamental matroid if and only if each block has a fundamental basis that includes a loop at every cut-vertex.

The above corollary helps us devise an algorithm to find out whether a given bicircular matroid $B(G)$ is fundamental. To do so, we check whether there is a loop at each cut-vertex. (We can use network flow algorithms to find whether a pair of vertices is contained in a cycle. This determines the blocks of $G$.) Then decompose $G$ into blocks, while suppressing series pairs, and apply the algorithm we discussed earlier. 


\section{Chapter 12}

\section{Bicircular and cobicircular matroids}

The intersection of bicircular and cobicircular matroids is another class of transversal and cotransversal matroids that is worthy of attention. In this chapter, we have made some progress towards characterising the matroids that belong to this class.

Definition 12.1.1. A whirly-swirl is a bicircular matroid $B(G)$ where $G$ has minimum degree at least three and has a cycle as the underlying simple graph where every parallel class has size at most two and no vertex is incident with more than one loop.

Figures 12.1 , 12.2 and 12.3 represent $G$ for some whirly-swirls $B(G)$. Note that all whirls and free swirls are members of this family.
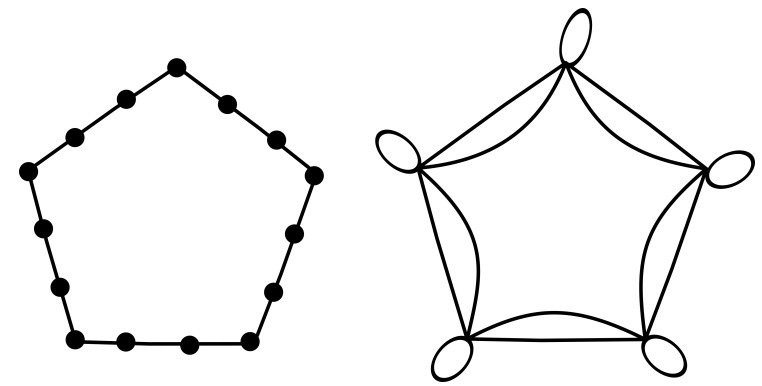

Figure 12.1: A whirly-swirl with every edge in a cycle graph doubled and every vertex incident with a loop 

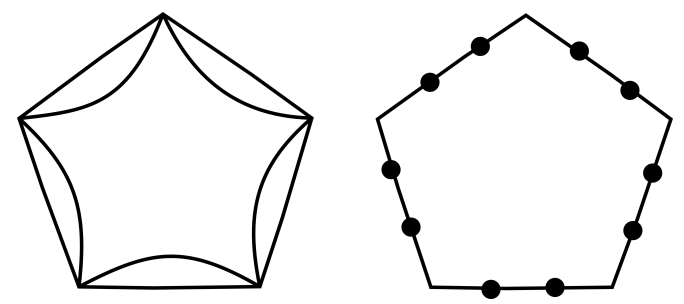

Figure 12.2: A whirly-swirl with every edge in a cycle graph doubled and no vertex incident with a loop. Note that this is also the rank-5 free swirl
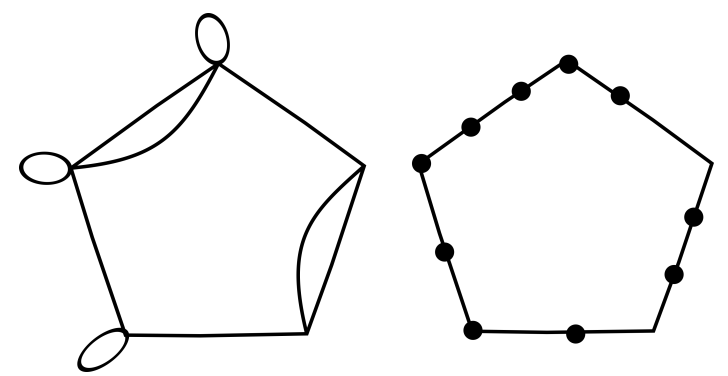

Figure 12.3: A whirly-swirl with some edges in a cycle graph doubled and some vertices incident with a loop

Clearly, whirly-swirls are bicircular matroids. To prove that they are cobicircular, we first denote the digons (two element cycles) of $G$ as $\left\{a_{i}, b_{i}\right\}$ where $1 \leq i \leq s$ for some positive integer $s$. Let the loops in $G$ be $l_{1}, \ldots, l_{t}$. Say $e_{1}, \ldots, e_{n}$ is the cyclic ordering of edges in $a 1, \ldots, a_{s}, l_{1}, \ldots, l_{t}$. Let $G^{*}$ be the graph with the underlying cycle that has edges $e_{1}, \ldots, e_{n}$, in cyclic order. The edges in every $\left\{a_{i}, b_{i}\right\}$ that is not part of the cycle $e_{1}, \ldots, e_{n}$ is now added as a parallel edge to the corresponding element in $G^{*}$. Hence every loop is now a link (not in a digon) in $G^{*}$. Now let $x$ be a link in $G$ that is not in a parallel class and is between $e_{i}$ and $e_{i+1}$. Then, $x$ is a loop in $G^{*}$ such that $x$ is incident with the vertex that is incident with $e_{i}$ and $e_{i+1}$. This construction also guarantees that every vertex in $G^{*}$ has degree at least three as the same is true in G. Figure 12.4 illustrates this fact.

In this figure, $\left\{a_{1}, b_{1}\right\}=\{a, b\},\left\{a_{2}, b_{2}\right\}=\{e, f\},\left\{l_{1}, \ldots, l_{4}\right\}=\{c, g, i, k\}$ and $\left(e_{1}, \ldots, e_{6}\right)=(a, c, e, g, i, k)$. Following the construction mentioned above, we get that $(a, c, e, g, i, k)$ is now the underlying cycle of $G^{*}$ and all loops in $G$ are now links in $G^{*}$ that are not in parallel classes. Also, $d$ is in between $c$ and $e$ in the cyclic order $e_{1}, \ldots, e_{6}$ and in $G^{*}, d$ is a loop at the vertex that is incident with $c$ and $e$. It is now easy to use our construction of $G^{*}$ to prove the following result, that is, $B\left(G^{*}\right)=B^{*}(G)$ and: 

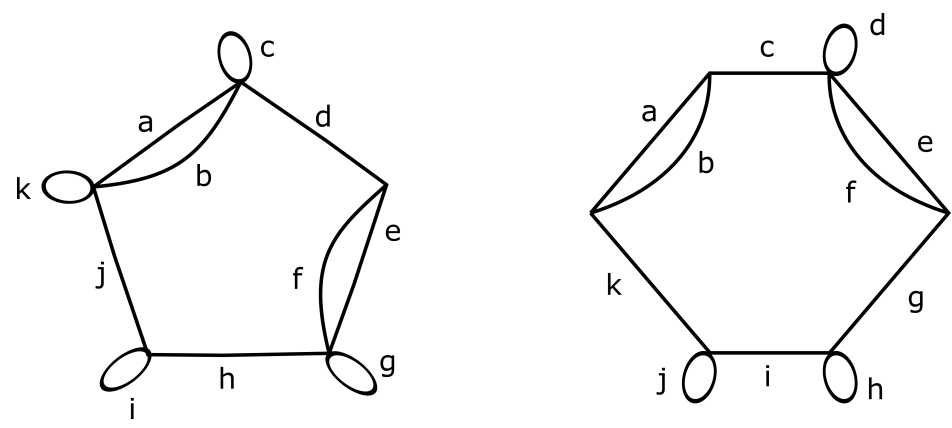

Figure 12.4: The underlying graph of a whirly-swirl on the left and its dual on the right

Lemma 12.1.2. A whirly-swirl is a bicircular and cobicircular matroid.

Now we are ready to formally state our conjecture:

Conjecture 12.1.3. There exists a positive integer $n$ such that when $|E(M)|>n$ and $M$ is 3-connected, bicircular and cobicircular, then $M$ is a whirly-swirl.

For small ranks we have examples of matroids that are both bicircular and cobicircular and yet do not belong to the whirly-swirl family. It is our firm belief that these examples will eventually fade away and whirly-swirls would be the only matroids that fit the category.

The list of uniform matroids that are bicircular and cobicircular: $U_{2,5}, U_{3,5}, U_{2,6}, U_{4,6}$ and $U_{3,6}$. All matroids that have less than six elements and are bicircular and cobicircular are either the uniform matroids mentioned above or a whirly-swirl. The six-element matroid that is neither uniform nor a whirly-swirl and lie in this intersection is $P_{6}$.

The 3-connected seven-element matroids that are both bicircular and cobicircular are listed in Figure 12.5. Note that the first two matroids do not belong to the class of whirly-swirls - we explain why in the following paragraph - whereas the last three matroids are whirly-swirls. This points in the direction of the conjecture being true.

To see that the first matroid in the list is not a whirly-swirl, we observe that any graphical representation will have a parallel class of four elements that corresponds to the four-element line. Assume that this is not the case. Then, the four-element line will be represented as a parallel class with loop at at least one end. But this will create triangles in the graph that do not 
exist in the matroid when the remaining three edges are added along with the third vertex. For the second matroid, we observe that representing two disjoint three-point lines will ensure that we have at least one parallel class of size three. Else, we have two parallel pairs sharing a vertex and one loop at each of the other end-vertices but this creates triangles in the graph that do not exist in the matroid. This implies that the matroid is not a whirly-swirl. Further, we have the following result that strengthens the conviction in the above mentioned conjecture being true.

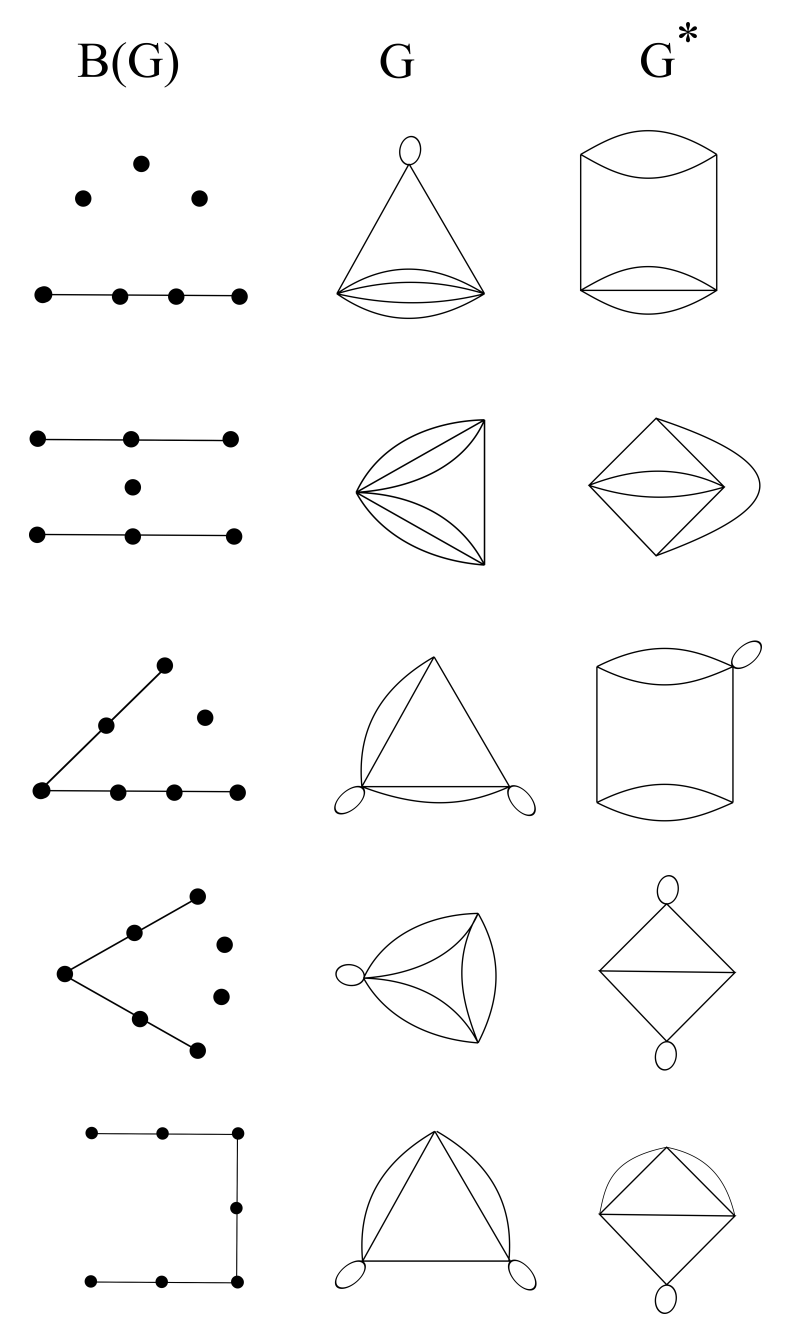

Figure 12.5: The first column is the geometric representation of each matroid, the second gives an underlying bicircular graph and the third column lists a graph of the dual.

Theorem 12.1.4. If $G$ has a loop at every vertex and $B(G)$ is 3-connected, bicircular and cobicircular, then $B(G)$ is a whirly-swirl. 
Proof. Let $B(G)$ be a bicircular and cobicircular matroid. Then for the sake of ease in notation, we use $G^{*}$ to denote a graph such that $B^{*}(G)=B\left(G^{*}\right)$. Let $B$ be the set of all loops in $G$. Then $B$ is a basis of $B(G)$ and for every $e$ not in $B$, the size of the fundamental circuit $C(e, B)$ is at most three. We arrive at this conclusion by observing that the fundamental circuit for every $e \in B$ is the loose handcuff that consists of the two loops at each end vertices of $e$ and $e$ itself. Also, $E-B$ is a basis of $B^{*}(G)$ and whenever $e \in E-B$, the fundamental cocircuit $C^{*}(e, B)$ has size at most three.

Now, every component of $G^{*}[E-B]$ has a unique cycle. Let $e$ be in $E-B$ and let $H$ be the component of $G^{*}[E, B]$ that contains $e$. We observe that $H \backslash e$ contains at least one acyclic component, say $H_{1}$. The fundamental cocircuit of $e$ consists of $e$ along with all elements of $B$ that are incident with $H_{1}$.

We claim that $H_{1}$ has at most two vertices. Assume otherwise. If $H_{1}$ has three or more leaves, then since every leaf must be incident with an edge of $C^{*}(e, E-B)$, at least one of these leaves has degree two in $G^{*}$. This is not possible as $B(G)$ and $B\left(G^{*}\right)$ are 3-connected. Then, if $H_{1}$ is a path with internal vertices, the same reasoning mentioned above applies and either a leaf or an internal vertex has degree two in $G^{*}$. Hence, the claim holds true and $H_{1}$ is either a single edge or a vertex.

Then, $H_{1} \cup e$ is either

(i) a digon,

(ii) a loop incident with a single edge,

(iii) a loop,

(iv) a path with two edges, or

(v) a single edge.

We prove that the first two scenarios and are valid and the others are impossible. We also describe what $H$ is in both these situations.

(i) If $H_{1} \cup e$ is a digon, then $H=H_{1} \cup e$. Assume otherwise. Then, $H$ is a digon attached to a path and by above reasoning, for any $e \in H$, 
$H-e$ should contain a path of length at most one. Since $e$ is either in the digon or in the path, $H$ has at most two edges. Thus, if $H_{1} \cup e$ is a digon, then $H$ is a digon too.

(ii) If $H_{1} \cup e$ is a loop incident with a single edge, $H=H_{1} \cup e$ again as argued above. Let $u$ and $v$ be the end vertices of $e$. Then, the sum of degrees of $u$ and $v$ is at most three. Let the loop be at vertex $u$. This implies that there are two links incident with $e$. If both the links are at vertex $v$, then $u$ is a cut-vertex of $G^{*}$. Else, if there is exactly one link at $v$, this is a degree two vertex in $G^{*}$ which is also impossible. Thus, $H$ is not a loop incident with an edge.

(iii) If $H_{1} \cup e$ is a loop, then $H$ is either a loop (and $H=H_{1} \cup e$ ) or $H$ is a loop with an edge incident with the loop-vertex, which we have already seen is impossible.

(iv) If $H_{1} \cup e$ is a path with two edges, then $H=H_{1} \cup e$ by above reasoning. Let the vertices in $H$ be $u, v$, and $w$, with $u$ adjacent with $v$ and $v$ adjacent with $w$. Then, since every cocircuit has size at most three, the sum of degrees of $u, v$, and $w$ minus the degrees contributed by $H$ cannot exceed three. Thus, $u, v$ and $w$ are connected in one of the ways as shown in Figure 12.6. If it is the case as in (a), then $u$ and $w$ have degree two, which contradicts the fact that $B\left(G^{*}\right)$ is 3-connected. The same is true for $u$ in (b). In the last case (c), $v$ is a cut-vertex in $G^{*}$, which produces the same contradiction as in cases (a) and (b).

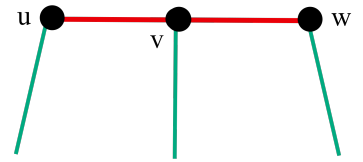

(a)

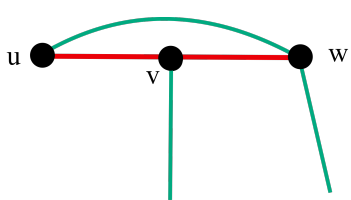

(b)

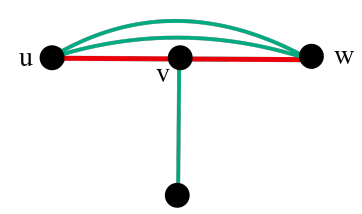

(c)

Figure 12.6: The red edges are in $H$ and the cyan edges are the other edges in $G^{*}$ that are incident with $u, v$ or $w$.

(v) If $H_{1} \cup e$ is a single edge, then $H$ is a cycle with a forest attached to it. Note that $H$ is not a digon since removal of any edge in a digon 
leaves a single edge and thus $H_{1} \cup e$ is a digon. Since $H_{1}$ has at most two vertices after the removal of any edge, $H$ in this case has to be a single edge attached to a loop. Then, $H$ has at most two more edges incident with it in $G^{*}$. But this implies that one of the end vertices of $H_{1} \cup e$ has degree two in $G^{*}$, which is impossible.

In conclusion, $H$ is either a loop or digon and thus $G^{*}[E-B]$ consists of loops and digons alone.

Now, in $G^{*}$, every vertex must have degree at least three, since $B\left(G^{*}\right)$ is 3-connected. Thus, a loop component of $E-B$ must be incident with at least two non-loop edges, or else $G^{*}$ has a cut-vertex. We observe that the loop is incident with exactly two non-loop edges as otherwise, the fundamental cocircuit has more than three elements. Similarly, both end vertices of a digon in $E-B$ are incident with exactly one non-loop edge. Therefore, we see that $G^{*}$ is obtained by 'stringing together' the loops and digons in $E-B$ by non-loop edges. Hence, $G^{*}$, and consequently $G$, are whirly-swirls. 
112 CHAPTER 12. BICIRCULAR AND COBICIRCULAR MATROIDS 


\section{Bibliography}

[1] Bonin, J. Lattice path matroids: The excluded minors. Journal of Combinatorial Theory, Series B 100 (09 2009).

[2] Bonin, J., KunG, J., AND DE Mier, A. Characterizations of transversal and fundamental transversal matroids. Electronic Journal of Combinatorics 18 (09 2010).

[3] Bonin, J. E. An introduction to transversal matroids. https://cpb-us-e1.wpmucdn.com/blogs.gwu.edu/dist/3/152/files/ 2016/04/TransversalNotes-2ltphpb.pdf, 2010.

[4] Bonin, J. E., AND DE Mier, A. The lattice of cyclic flats of a matroid. Annals of Combinatorics 12 (2005), 155-170.

[5] Bonin, J. E., AND DE MieR, A. Lattice path matroids: Structural properties. European Journal of Combinatorics 27, 5 (2006), 701 - 738.

[6] Bonin, J. E., DE Mier, A., AND Noy, M. Lattice path matroids: enumerative aspects and Tutte polynomials. J. Comb. Theory, Ser. A 104 (2002), 63-94.

[7] BRUALDI, R. A. On fundamental transversal matroids. Proceedings of the American Mathematical Society 45, 1 (1974), 151-156.

[8] Chen, R., And Whittle, G. On recognizing frame and lifted-graphic matroids. Journal of Graph Theory 87 (2016), 72-76.

[9] Diestel, R. Graph Theory (Graduate Texts in Mathematics). Springer, August 2005.

[10] EDMONDS, J., AND FulKerson, D. Transversals and Matroid Partition. Memorandum (Rand Corporation). Rand Corporation, 1965. 
[11] Franchella, M. On the origins of Dénes König's infinity lemma. Archive for History of Exact Sciences 51, 1 (Mar 1997), 3-27.

[12] FunK, D., MAYHeW, D., AND NeWman, M. Tree automata and pigeonhole classes of matroids - I. arXiv e-prints (2019), arXiv:1910.04360.

[13] FunK, D., MAYHeW, D., AND NEWMAN, M. Tree automata and pigeonhole classes of matroids - II. arXiv e-prints (2019), arXiv:1910.04361.

[14] Geelen, J., Gerards, B., AND Whittle, G. Solving Rota's conjecture. Notices of the American Mathematical Society 61 (08 2014), 736.

[15] Geelen, J. F., Gerards, A., And Whittle, G. Branch-width and well-quasi-ordering in matroids and graphs. Journal of Combinatorial Theory, Series B 84, 2 (2002), 270 - 290.

[16] Hall, P. On representatives of subsets. Journal of the London Mathematical Society s1-10, 1 (1935), 26-30.

[17] Hausmann, D., AND KorTe, B. Lower bounds on the worst-case complexity of some oracle algorithms. Discrete Mathematics 24 (12 1978), 261-276.

[18] Higman, G. Ordering by divisibility in abstract algebras. Proceedings of the London Mathematical Society s3-2, 1 (1952), 326-336.

[19] HLINĚNÝ, P. On matroid properties definable in the MSO logic. In Mathematical Foundations of Computer Science 2003 (Berlin, Heidelberg, 2003), Springer Berlin Heidelberg, pp. 470-479.

[20] Jensen, P. M., AND Korte, B. Complexity of matroid property algorithms. SIAM Journal on Computing 11, 1 (1982), 184-190.

[21] KÖNIG, D. Über eine schlussweise aus dem endlichen ins unendliche. Acta Sci. Math.(Szeged) 3, 2-3 (1927), 121-130.

[22] Matthews, L. R. Bicircular Matroids. The Quarterly Journal of Mathematics 28, 2 (06 1977), 213-227. 
[23] MAtúš, F. Classes of matroids closed under minors and principal extensions. Combinatorica 38, 4 (Aug. 2018), 935-954.

[24] Mayhew, D., NeWman, M., And Whittle, G. On excluded minors for real-representability. Journal of Combinatorial Theory, Series B 99, 4 (2009), $685-689$.

[25] Mayhew, D., Newman, M., And Whittle, G. Yes, the "missing axiom" of matroid theory is lost forever. Transactions of the American Mathematical Society 370, 8 (2018), 5907-5929.

[26] Mayhew, D., Whittle, G., And NeWman, M. Is the missing axiom of matroid theory lost forever? Quarterly Journal of Mathematics 65, 4 (2014), 1397-1415.

[27] Nash-Williams, C. S. J. A. On well-quasi-ordering finite trees. Proc. Cambridge Philos. Soc. 59 (1963), 833-835.

[28] OXLey, J. Matroid Theory. Oxford University Press, 2011.

[29] Oxley, J., Prendergast, K., And Row, D. Matroids whose ground sets are domains of functions. J. Austral. Math. Soc. Ser. A 32, 3 (1982), 380-387.

[30] Oxley, J., Semple, C., AND Whittle, G. Maintaining 3-connectivity relative to a fixed basis. Adv. in Appl. Math. 41, 1 (2008), 1-9.

[31] Simões PereirA, J. M. S. On subgraphs as matroid cells. Mathematische Zeitschrift 127, 4 (Dec 1972), 315-322.

[32] Whitney, H. On the abstract properties of linear dependence. American Journal of Mathematics 57, 3 (1935), 509-533.

[33] Whittle, G., AND Williams, A. On preserving matroid 3-connectivity relative to a fixed basis. European Journal of Combinatorics 34, 6 (2013), 957 - 967. 